\title{
High resolution biostratigraphy of the Tournaisian-Viséan boundary interval in the North Staffordshire Basin and correlation with the South Wales-Mendip Shelf
}

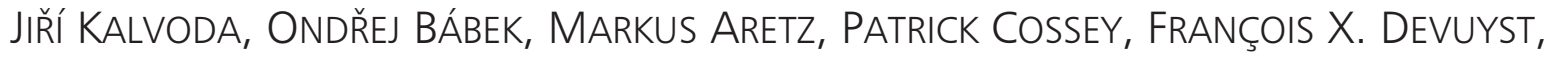 \\ SIMON HARGREAVES \& JOHN NUDDS
}

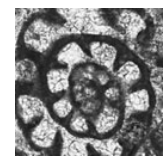

\begin{abstract}
A study of three carbonate sections in the North Staffordshire Basin and two in the South Wales-Mendip Shelf yielded so far the richest foraminiferal associations at the Tournaisian-Viséan boundary in Western Europe enabling a very good correlation with the stratotype area in South China. The biostratigraphic study was refined by sedimentological and gamma-spectrometrical data in order to define and calibrate the high resolution biostratigraphy across the boundary interval. The study illustrates the cryptic entry of the stratigraphically important species of the Chadian index genus Eoparastaffella which is abundant in photozoan facies. Across the Tournaisian-Viséan boundary, changes in the character of the foraminiferal associations reflect the gradual change from heterozoan dominated late Tournaisian ramps to Viséan photozoan dominated platforms. The combined application of biostratigraphic and sedimentological methods and gamma ray manifestation of the mid Avonian sequence boundaries have proved useful in the identification of the Tournaisian-Viséan boundary in Carboniferous successions on either side of the Wales-Brabant Massif and has widespread applicability for successions of a similar age across Western Europe. - Key words: Tournaisian, Viséan, foraminiferal biostratigraphy and ecology, carbonate sedimentology, gamma-ray spectrometry.
\end{abstract}

Kalvoda, J., BÁBek, O., AREtZ, M., Cossey, P., Devuyst, F.X., HARgreaves, S. \& NudDS, J. 2012. High resolution biostratigraphy of the Tournaisian-Viséan boundary interval in the North Staffordshire Basin and correlation with the South Wales-Mendip Shelf. Bulletin of Geosciences 87(3), 497-541 (28 figures). Czech Geological Survey, Prague. ISSN 1214-1119. Manuscript received January 25, 2012; accepted in revised form May 23, 2012; published online August 15, 2012; issued September 28, 2012.

Jiři Kalvoda \& Ondřej Bábek, Masaryk University Brno, Department of Geological Sciences, Czech Republic; dino@sci.muni.cz・Ondřej Bábek, Palacký University Olomouc, Department of Geology, Czech Republic・Markus Aretz, Université de Toulouse (UPS), LMTG (OMP), France $\bullet$ Patrick Cossey \& Simon Hargreaves, Faculty of Sciences, Staffordshire University, Stoke-on-Trent, England $\bullet$ François X. Devuyst, Carmeuse Lime and Stone, Technology Center, Pittsburgh PA 15225, USA • John Nudds, School of Earth, Atmospheric and Environmental Sciences, University of Manchester, England

In accordance with the definition of the base of the Viséan in Belgium, foraminiferal genus Eoparastaffella was used to define the base of the Chadian regional stage in Britain (George et al. 1976). However, subsequent workers have failed to repeat this foraminiferal record at the Chatburn stratotype in the Craven Basin (Fewtrell et al. 1981a, b; Riley 1990, 1993). Subsequently however refinements in our understanding of foraminiferal lineages across the Tournaisian-Viséan (T-V) boundary have led to the redefinition of the base of the Viséan and its stratotype changed. The base of the Viséan is now defined by first appearance of the foraminifer Eoparastaffella simplex within the Eoparastaffella ovalis - Eoparastaffella simplex lineage, and a section at Pengchong near Liuzhou in Guangxi, southern
China, has been chosen as the GSSP; a redefinition originally proposed by Devuyst et al. (2003) and later ratified by the ICS and IUGS (Work 2008). Consequently, the Chadian in its original definition now comprises the T-V boundary interval starting in the last Tournaisian foraminiferal zone MFZ8 and ending at the top of the earliest Viséan zone MFZ9 (Hance \& Devuyst in Poty et al. 2006, Hance et al. 2011).

Taking into the account the problems connected with the Chadian in the Craven Basin we tried to identify the recent concept of the T-V boundary in the North Staffordshire Basin where excellent outcrops occur at Brown End Quarry, Ladyside Wood and Ossom's Hill in a deeper ramp setting (Aitkenhead et al. 1985, Chisholm et al. 1988). 


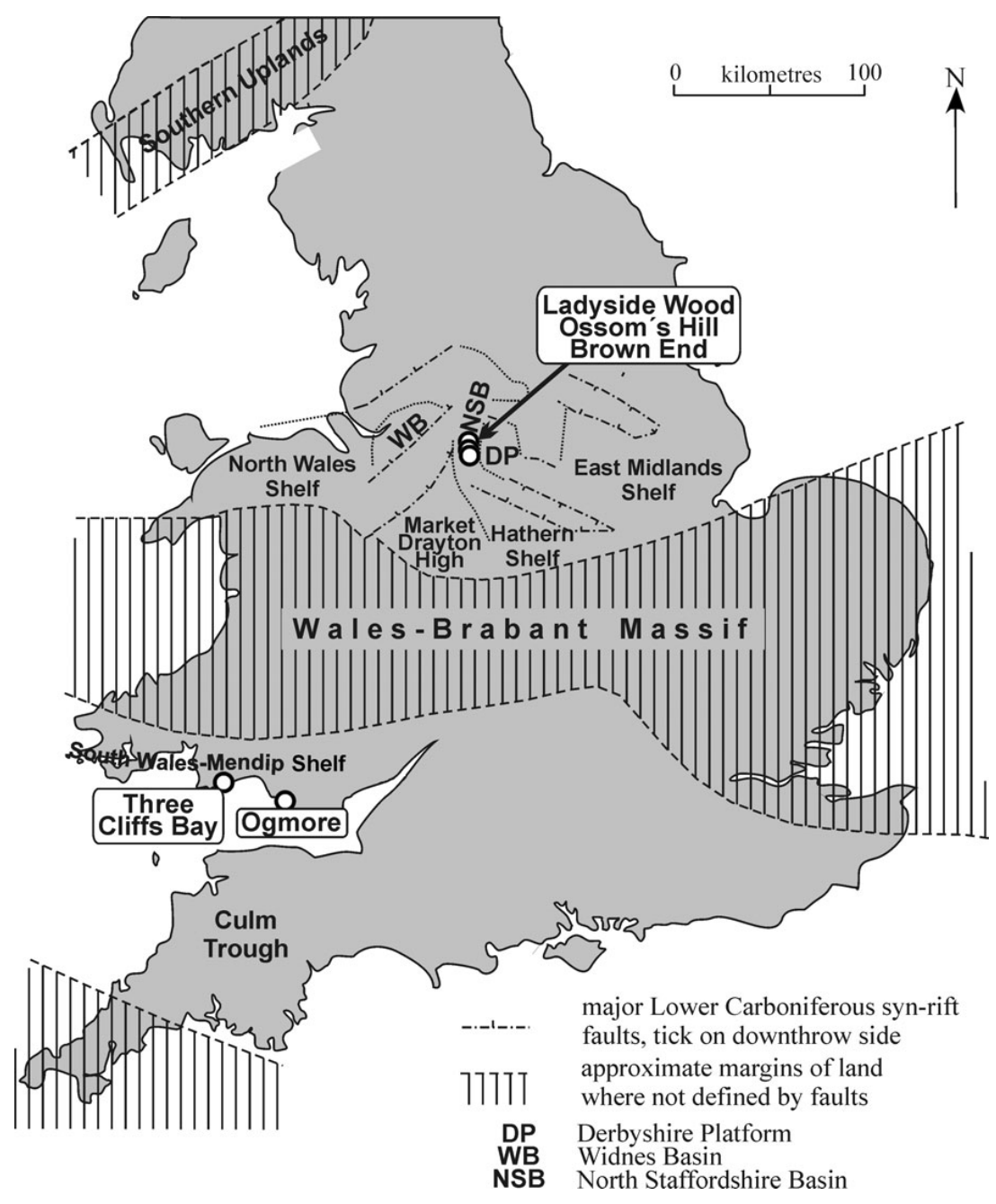

Figure 1. Location of the studied sections on the flanks of the Wales-Brabant Massif (modified after Cossey et al. 2004).

For comparison, the same boundary was investigated in some fine sections on the South Wales-Mendip Shelf where sections with distinct sequence boundaries occur at Three Cliffs Bay and Ogmore in a shallow ramp setting (Adams et al. 2004, Wright 1986).

As originally outlined by Ramsbottom (1973, 1981) and recently confirmed by Hance et al. (2001, 2002), the $\mathrm{T}-\mathrm{V}$ boundary represents an important sequence boundary around the Wales-Brabant Massif. In this respect gammaray spectrometry (GRS) has already proved as a useful tool in tracing sea-level oscillations, supplementing biostratigraphy and improving dramatically the resolution of stratigraphic correlations (Bábek et al. 2010, Delius et al. 2001, Doveton 1994, Hladil et al. 2006, Rider 1999, Thibal et al. 1999). The successful application of this method for correlating the T-V boundary interval in the Dublin Basin, Ireland was clearly demonstrated by Kalvoda et al. (2011). Although this Irish study demonstrated the utility of foraminifera in high resolution stratigraphy it also demonstrated that foraminiferal assemblages were strongly facies controlled and that detailed microfacies study is required in order to realise their true stratigraphic potential. Consequently the present study aims at improving the resolution of the biostratigraphy and sequence stratigraphy of late Tournaisian and early Viséan rocks of the North Staffordshire Basin and the South Wales-Mendip Shelf by combining high-resolution foraminifer biostratigraphy with sedimentological and petrophysical methods (gamma ray spectrometry).

\section{Material and methods}

Five sections were described, bed by bed logged and sampled for foraminifer biostratigraphy and thin section analysis. Petrographic analysis was performed on a large number of standard size $(27 \times 46 \mathrm{~mm})$ thin sections. Semiquantitative percentages of carbonate and non-carbonate constituents were recorded, using the visual comparison charts of Baccelle \& Bosellini (1965). Only the sand and gravel size grains (allochems and detrital grains) were considered, fine-grained matrix and cements were omitted. 
Results are presented as percentages of individual compositional groups from the total count of grains.

A first series of thin sections for foraminifer study was typically made and examined to identify the richest levels. Additional numerous thin sections (over 700) were cut in the best beds. Foraminifers were studied with a high-magnification binocular microscope (Nikon 80i) in transmitted light at magnifications ranging from $20 \times$ to $400 \times$. The best specimens were photographed with a digital camera (Nikon DXM) and a database was created using Nikon NIS Elements software.

\section{Sedimentology}

\section{North Staffordshire Basin}

\section{Brown End section}

The Brown End Quarry section is $\sim 65 \mathrm{~m}$ thick and comprises carbonates of the Milldale Limestone and the Hopedale Limestone (uppermost Tournaisian to lower Viséan). Graded, sometimes amalgamated, bioclastic turbidites (calcarenites to calcirudites) with abundant echinoderms, corals and brachiopods and frequent Zoophycos trace fossils, alternating with grey to reddish marls (Milldale Limestone), predominate in the basal part of the section (65 to $47 \mathrm{~m}$ ). The overlying coarsening-upward succession (47 to $30 \mathrm{~m}$ ) comprises calcisiltites and fine-grained calcarenites with chert nodules, alternating with thin marl interlayers and scarce graded crinoidal calciturbidite beds. This succession is overlain by a sequence of very thick beds of coarse-grained calcarenites to fine calcirudites (30 to $24 \mathrm{~m}$ ), a fining-upward succession of graded and parallellaminated carbonate turbidites ( 24 to $17 \mathrm{~m}$ ) and finally by calcisiltites to fine-grained calcarenites with chert nodules and marl interlayers (17 to $8.5 \mathrm{~m}$ ). The topmost part of the profile ( 8.5 to $0 \mathrm{~m}$, Hopedale Limestone) is composed of thick beds of coarse-grained, graded and parallel and/or cross-laminated calcarenites and carbonate breccias with abundant bioclasts. Massive micritic carbonate buildups with frequent stromatactis and open-marine fauna ("knoll reefs" or "Waulsortian" carbonate mudmounds) are exposed in the Tournaisian part of the Brown End Quarry, below the measured section (Aitkenhead et al. 1985, Cossey et al. 1995). Two erosional surfaces are documented at the section, one at the base of a thick layer of massive coarse-grained crinoidal calcarenite $(\sim 28.5 \mathrm{~m})$ and another one close to the base of the Hopedale Limestone $(\sim 3.3 \mathrm{~m}$, Fig. 2; Cossey et al. 1995, 2004).

The carbonates at the Brown End section can be classified as fine-grained to very coarse-grained wacke-packstones, packstones and rare well-sorted grainstones. Skeletal allochems (from 12 to $36 \%$ vol.) predominate over non-skeletal ones (from 1 to $30 \%$ vol.). Skeletal grains include abundant crinoids and other echinoderm fragments, green algae (dasyclad algae, palaeoberesellids and related forms), unilocular and plurilocular foraminifers, calcispheres, sponge spicules, bryozoan fragments, brachiopods, bivalves, ostracods, less abundant corals, trilobites, gastropods, cyanobacteria (Girvanella) and unidentified grains (Fig. 2). Non-skeletal grains include very abundant peloids and aggregate grains and rare intraclasts and ooids.

Crinoids, bryozoans, brachiopods and sponge spicules are quite abundant in the bottom part of the section (Tournaisian, 65 to $48 \mathrm{~m}$ ). The relative abundance of crinoids then decreases in favour of peloids, which become the dominant compositional group for the remainder of the section (48 to $0 \mathrm{~m}$; uppermost Tournaisian to lower Viséan). There is an ever-increasing abundance of green algae (dasyclad algae, palaeoberesellids and related forms) and multichambered foraminifers starting approximately from the base of the Viséan ( $32 \mathrm{~m}$ ) up to the top of the section while the relative abundance of bryozoans and sponge spicules decreases in the same interval. This upper part of the section is associated with frequent allochem micritization.

\section{Ladyside Wood and Ossom's Hill section}

The Ladyside Wood section is $~ 56 \mathrm{~m}$ thick and comprises carbonates of the Milldale Limestone and the Ecton Limestone (uppermost Tournaisian to lower Viséan; Fig. 2). At the base of the section ( $0-2 \mathrm{~m})$, massive micritic carbonates with stromatactis and abundant echinoderms, bryozoans, ostracods and open-marine fauna are exposed. They are interpreted as "knoll reefs" or "Waulsortian" carbonate mudmounds (Aitkenhead et al. 1985, Cossey et al. 1995). They are overlain $(2-\sim 56 \mathrm{~m})$ by a succession of graded, parallel laminated fine- to coarse grained calcarenites with occasional scours, ripple-cross lamination, wavy lamination, soft-sediment deformation structures and abundant echinoderms, peloids, dasyclad algae and foraminifers, which alternate with calcisilitites, calcilutites and marls, sometimes cherty, with abundant ostracods and sponge spicules. The sediments are interpreted as proximal and distal carbonate turbidites deposited in outer ramp environment (Aitkenhead et al. 1985, Cossey et al. 2004).

The carbonates at the Ladyside Wood section range from wackestones through to packstones and usually well-sorted grainstones. Skeletal allochems (from 16 to $37 \%$ vol.) predominate over non-skeletal ones (from 0 to $18 \%$ vol.). Skeletal grains include abundant crinoids and other echinoderm fragments, green algae (dasyclad algae, palaeoberesellids and related forms), unilocular and plurilocular foraminifers, and less abundant calcispheres, 

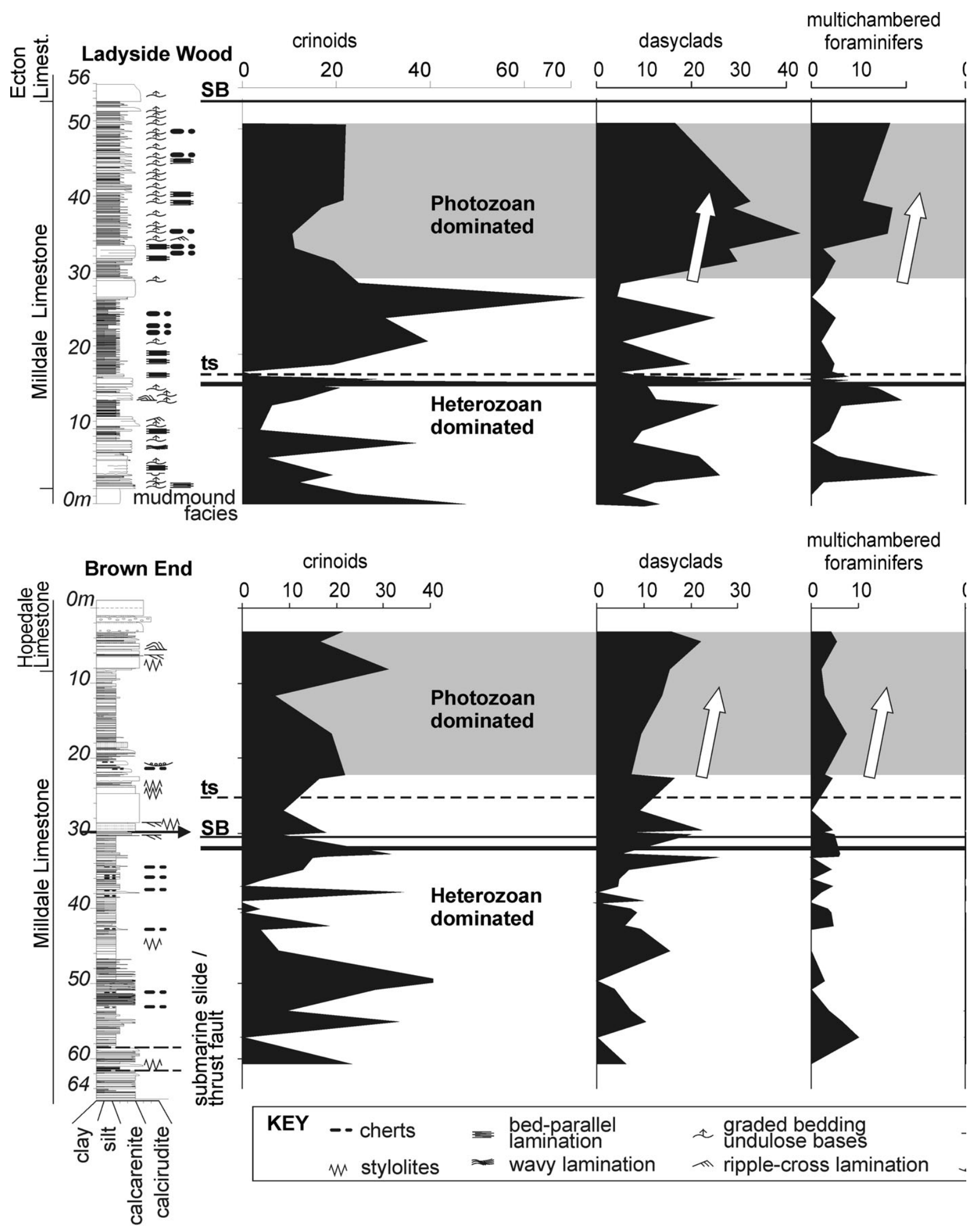

Figure 2. Stratigraphic trends in selected compositional groups (percentages from total rock volume based on visual estimates, using comparative charts) and correlation of the Brown End and Ladyside Wood sections in the North Staffordshire Basin. 

multichambered
foraminifers

bryozoans ostracods sponge spicules

peloids

Ladyside Wood

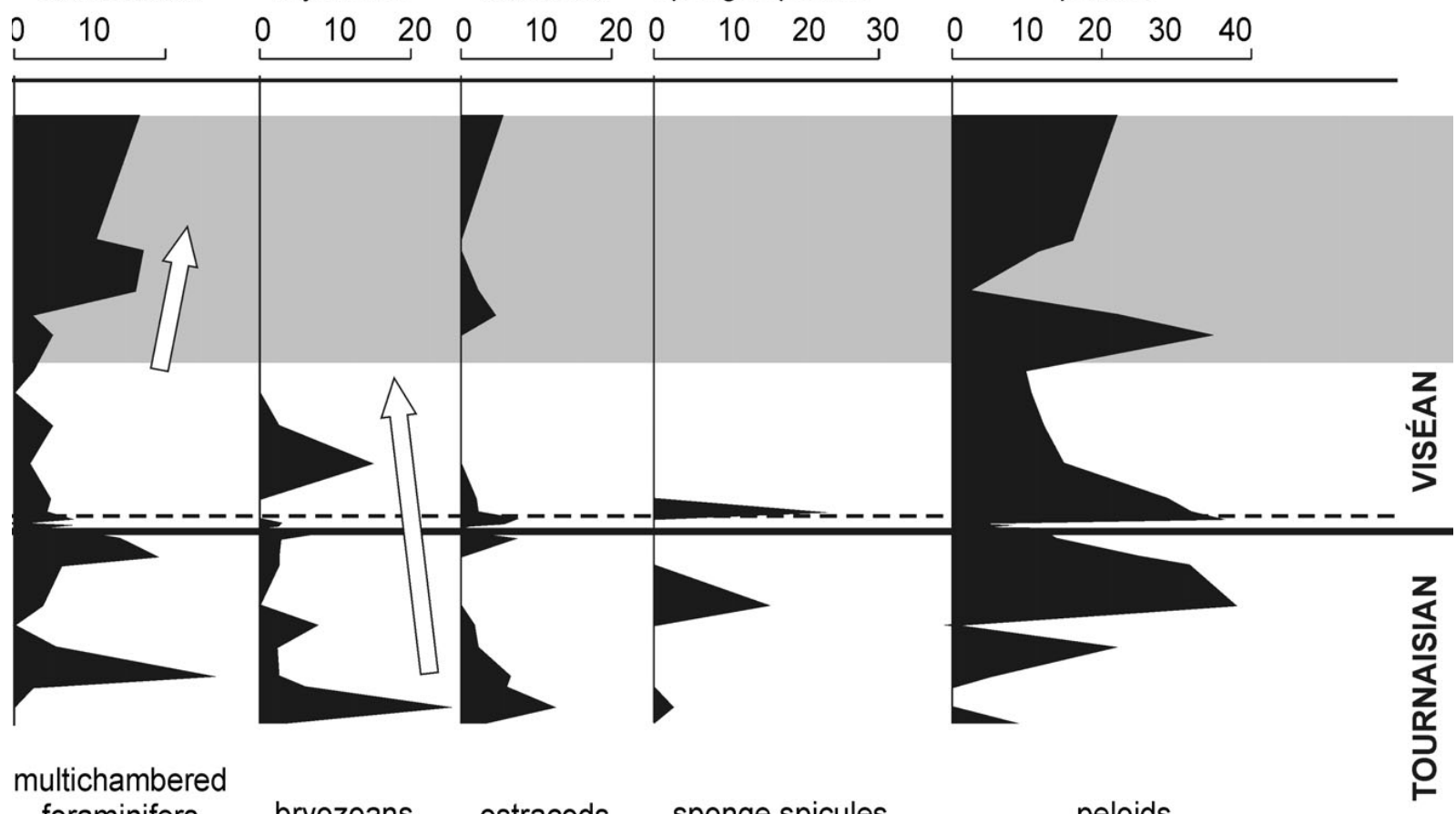

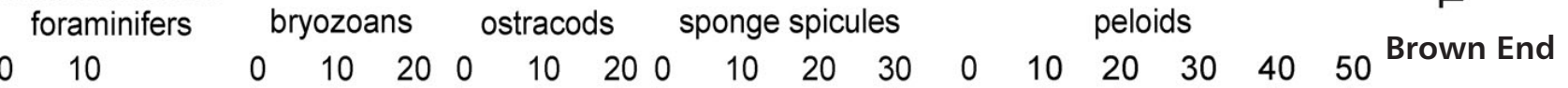

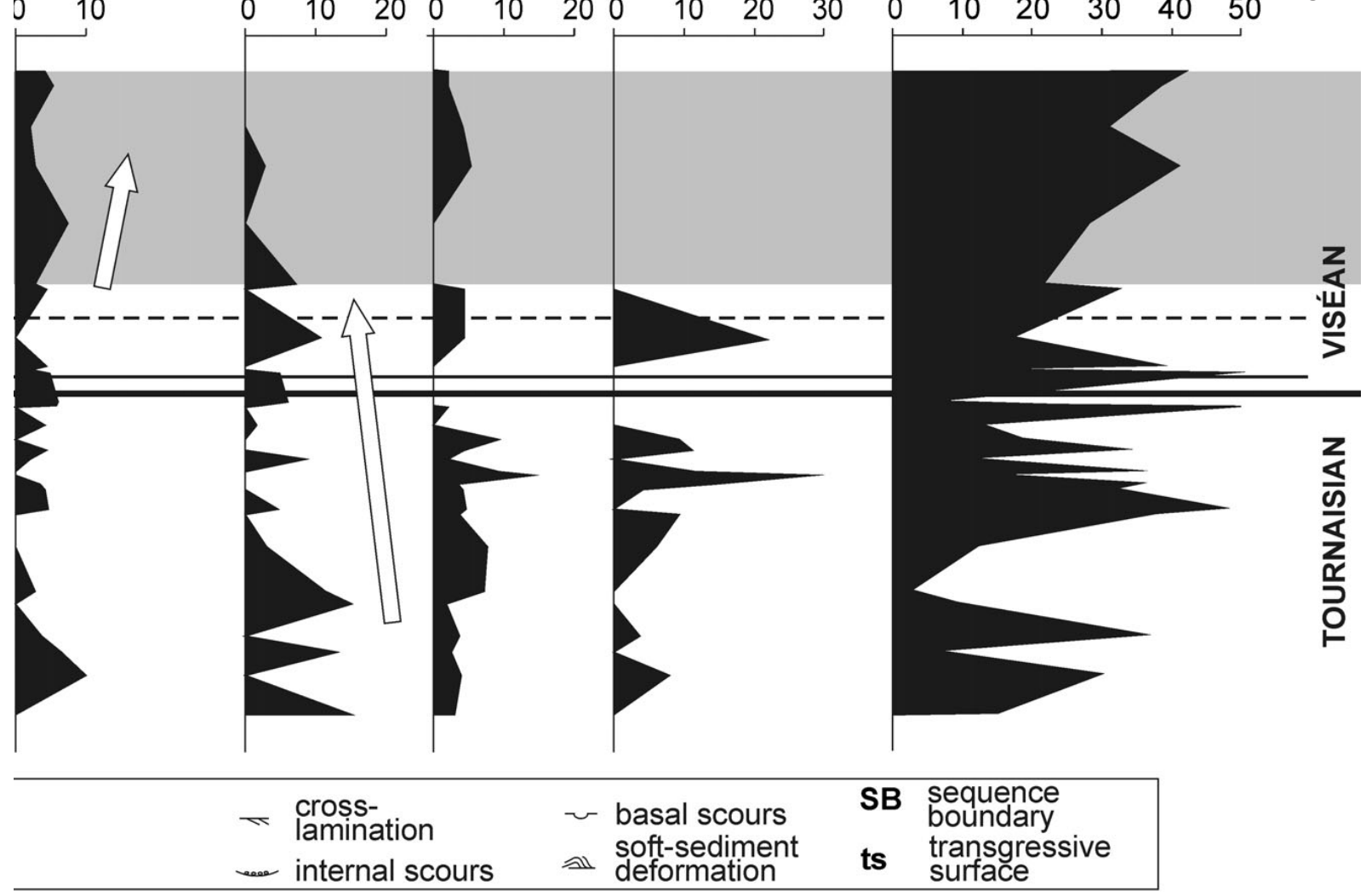

Figure 2. Continued. 


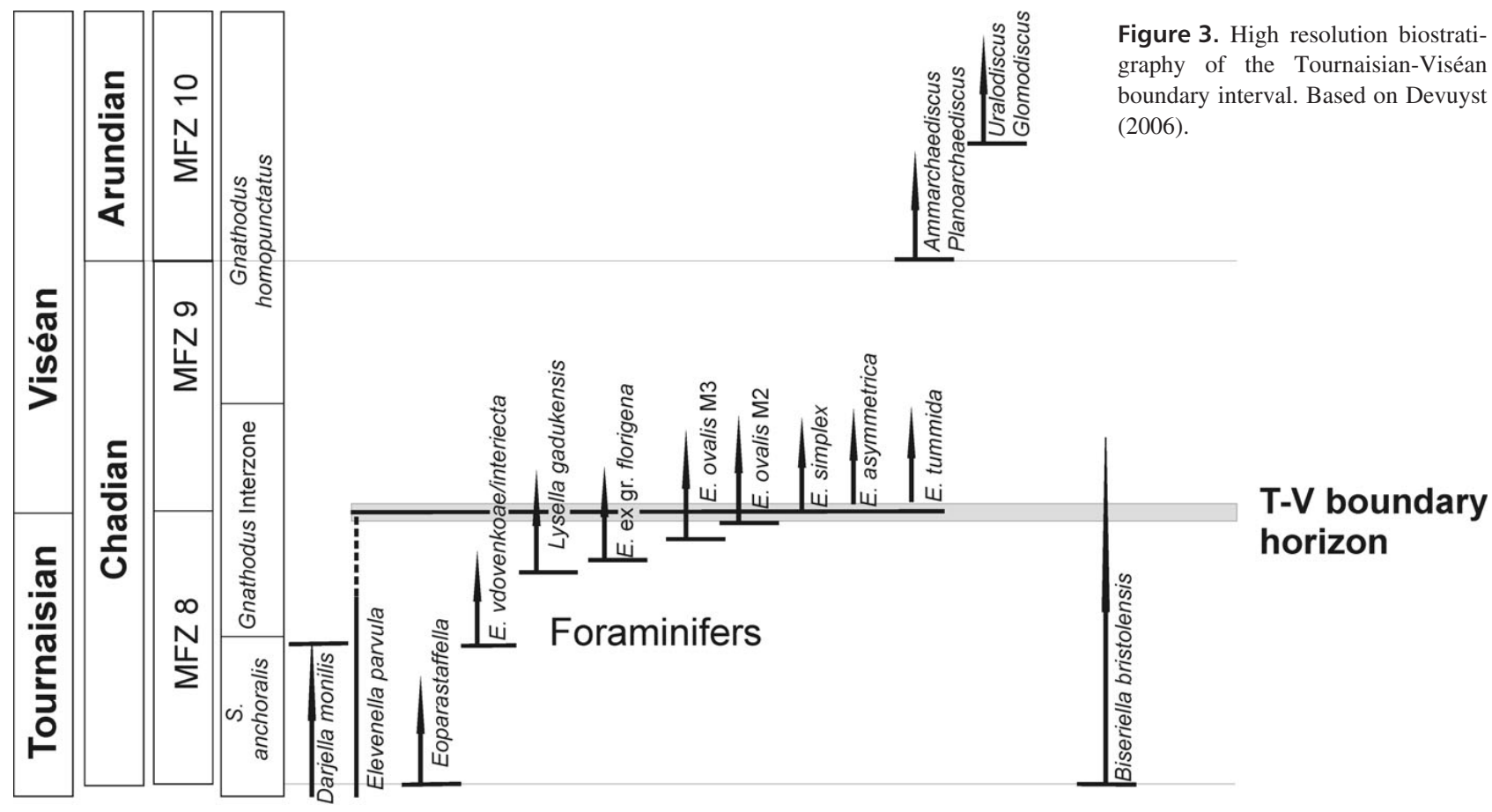

sponge spicules, bryozoan fragments, brachiopods, bivalves, ostracods, corals, trilobites, gastropods, cyanobacteria (Girvanella) and unidentified grains (Fig. 2). Non-skeletal grains include very abundant peloids and aggregate grains and intraclasts. Ooids are almost absent. The allochem composition and the vertical compositional trends at the Ladyside Wood section are similar to those of the Brown End section (Fig. 2). The most prominent features include the sudden composition change at the upper boundary of the mudmound facies (upper Tournaisian) and then an increase in the proportion of dasyclad algae and foraminifers in the top part of the section ( 30 to $\sim 50 \mathrm{~m}$, lower Viséan).

The Ossom's Hill section is approximately $42 \mathrm{~m}$ thick and comprises carbonates of the Milldale Limestone $(0$ to $31 \mathrm{~m})$ and the Ecton Limestone ( 31 to $42 \mathrm{~m})$. The whole section is early Viséan in age. The base of the section $(0-9 \mathrm{~m})$ exposes fine-grained calcarenites and calcisiltites with thin, graded, calcarenite beds, abundant cherts and trace fossils (Chondrites, Zoophycos). The proportion of the graded calcarenites increases upsection. This succession gradually passes upward into a thickening and coarsening upward succession of predominantly graded calcarenites (calciturbidites) with rare chert nodules $(9-21 \mathrm{~m})$. The overlying part $(21-30.5 \mathrm{~m})$ is fining and thinning-upward, comprising graded calcarenites, calcisiltites and mudstones with cherts and trace fossils. The top part of the section ( 30.5 to $42 \mathrm{~m}$ ) comprises relatively thick (up to $60 \mathrm{~cm}$ ) beds of graded, coarse- to medium grained calcarenites (proximal calciturbidites). The Ossom's Hill section was not studied in detail sedimentologically as its base is already Viséan in age and it shows a similar development to the more complete section at Ladyside Wood.

\section{South Wales - Mendip Shelf}

\section{Three Cliffs Bay and Ogmore sections}

The studied succession in Three Cliffs Bay (Fig. 7) starts in Courceyan to Chadian bioclastic limestones of the Friars Point Limestone (formerly Tears Point Limestone, see Waters et al. 2011) and comprises argillaceous, burrowed beds with a fully marine biota, but lacking very shallow forms (dasycladaceans). It exhibits sharp based, normally graded and hummocky stratified calcarenite beds, sometimes with shell lags. The sedimentology was discussed in detail by Wu (1982), Wright (1986) and Ramsay (1987) and was interpreted as a succession deposited in marine subtidal environments ranging from depths below the zone of significant wave and current activity to depths above storm-wave base. The gradual passage from the bioclastic Friars Point Limestone into the Chadian Gully Oolite (formerly Caswell Bay Oolite, see Waters et al. 2011) represents a shallowing-up (regressive) sequence. The shoreface Gully Oolite represents barrier islands and beaches, with preserved shoreface sequences, which developed beach-ridge plains on various scales (Ramsay 1987, Burchette et al. 1990). The Caswell Bay Mudstone, overlies the karstic and palaeosol-covered top of the Gully Oolite (Riding \& Wright 1981, Wright 1986) and is erosively overlain by bioclastic lower-shoreface deposits of the Arundian High 
Jiři Kalvoda et al. • High resolution biostratigraphy of the Tournaisian-Viséan boundary interval in the North Staffordshire Basin

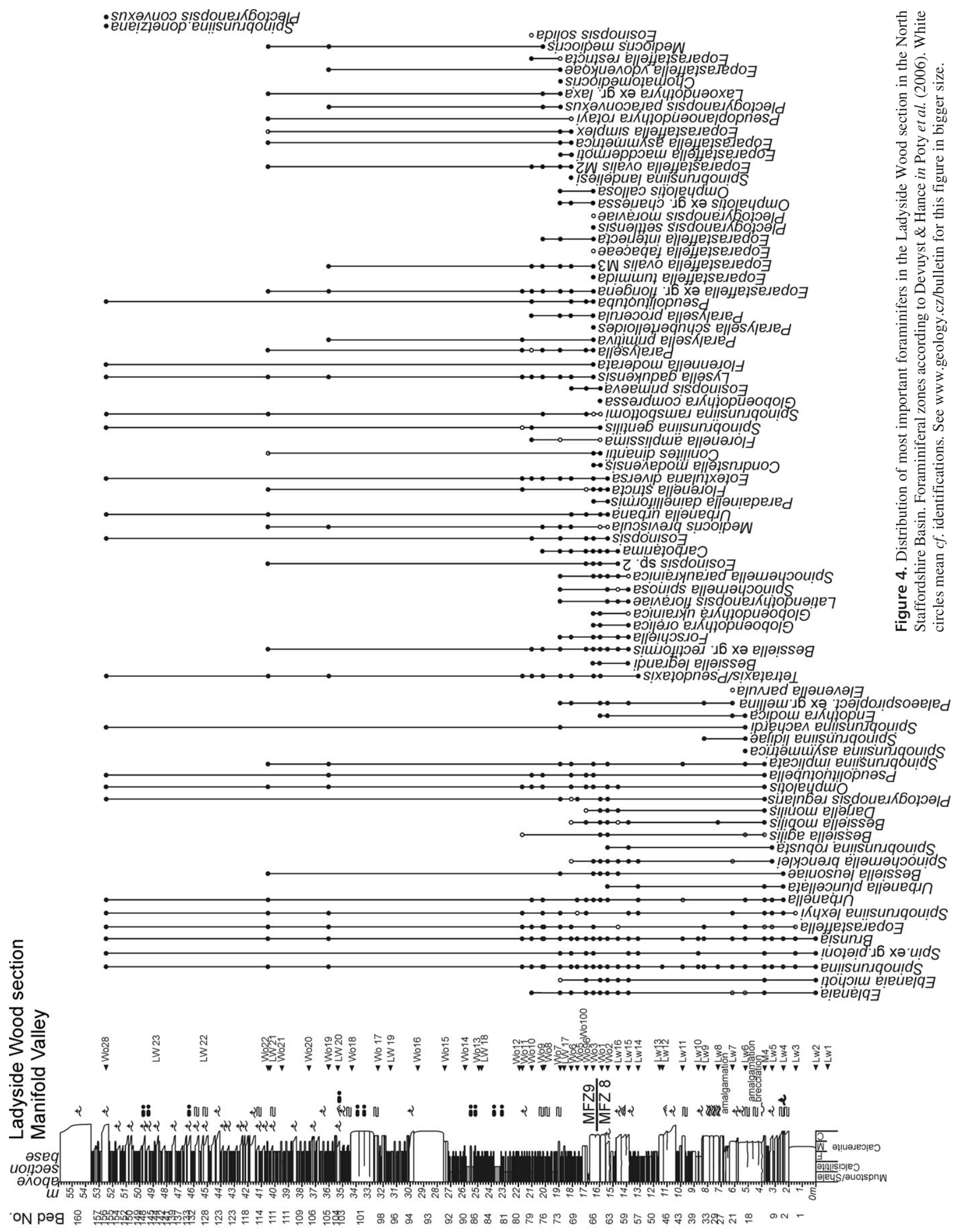

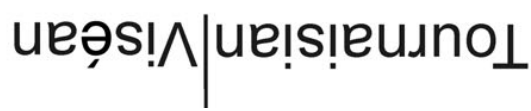




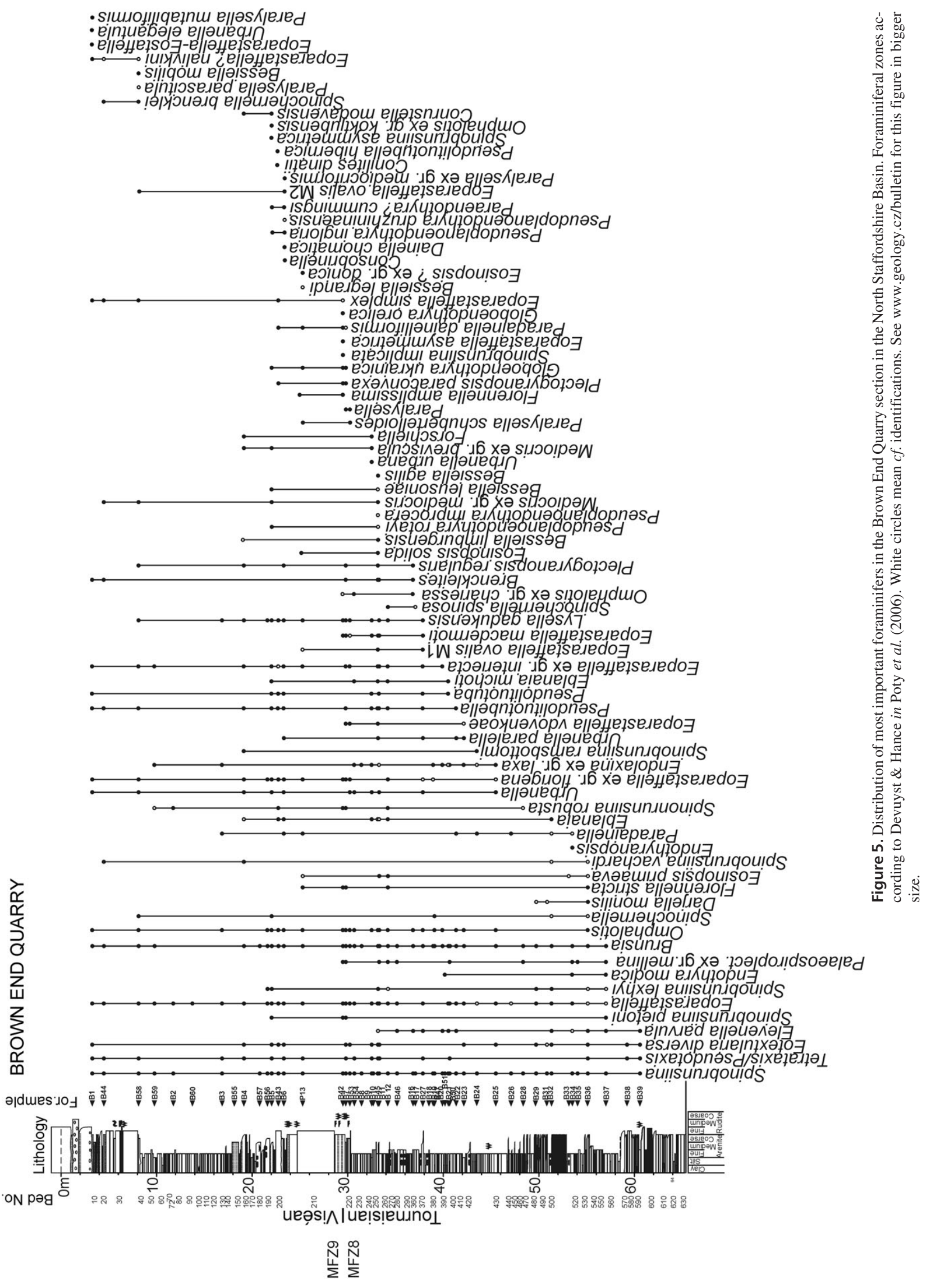




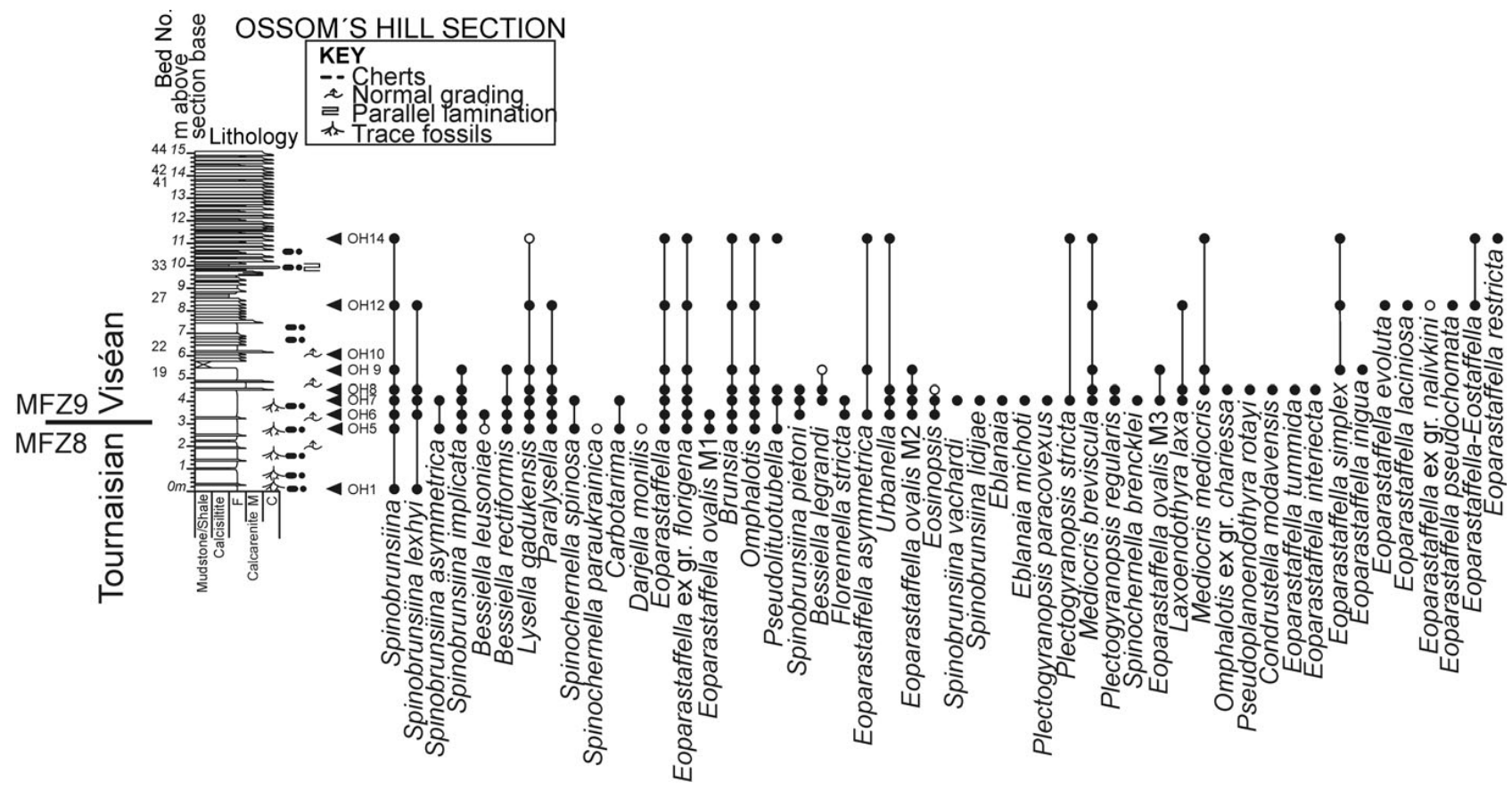

Figure 6. Distribution of most important foraminifers in the Viséan (MFZ9) of the Ossom's Hill section in the North Staffordshire Basin. Foraminiferal zones according to Devuyst \& Hance in Poty et al.(2006). White circles mean $c f$. identifications. See www.geology.cz/bulletin for this figure in bigger size.

Tor Limestone (Riding \& Wright 1981, Wright 1986, Ramsay 1987).

The Ogmore section (SS 86857425, Fig. 8) is $\sim 25 \mathrm{~m}$ thick and comprises a comparable sequence to that at Three Cliffs Bay, recording the passage from the upper part of the Gully Oolite into the Caswell Bay Mudstone and overlain by the High Tor Limestone (Chadian to Arundian substages). The basal part of the section (0 to $7.75 \mathrm{~m}$ ) consists of thickly-bedded, poorly-sorted, skeletal-peloidal packestones of the Gully Oolite, with some finer grained packestones and in places wackestones. Their lithology is similar to the Gully Oolite at Three Cliffs Bay, but the lack of typical oolites suggests deeper water deposits. Waters (1984) records a thickness of $83 \mathrm{~m}$ of Gully Oolite in the St Lythan's borehole (ST 10547290) where it comprises four shoaling upward cycles, the top two becoming subaerial. A major bedding plane at Ogmore records the passage into the overlying Caswell Bay Mudstone (7.75 to $16.75 \mathrm{~m}$ ) comprising thinner-bedded wackestones with coarser shelly debris, small crinoid ossicles, occasional zaphrentid corals and pleurotomarid gastropods. Some bedding planes are bioturbated with sub-horizontal Thalassinoides burrows. At $\sim 13.5 \mathrm{~m}$ a palaeokarst and palaeosol with calcrete is developed and is followed by crinoidal wackestones with large pleurotomarid gastropods, which are overlain (15.75 to $16.75 \mathrm{~m}$ ) by a soft, poorly-consolidated mudstone. As in Three Cliffs Bay the mudstone is followed by bioclastic, lower-shoreface deposits of the High Tor Limestone, with a rich fauna of rugose and tabulate corals, thick-shelled brachiopods and gastropods.

\section{Biostratigraphy}

The definition of the regional stages for the British Isles and Ireland proposed by George et al. (1976) capitalized on the progress of foraminiferal biostratigraphy introduced in Western Europe by Raphael Conil (Conil \& Lys 1964, Conil et al. 1977) who subsequently progressively applied his zonation to British and Irish regional stages (Conil et al. 1980). Later, Fewtrell et al. (1981b) documented foraminiferal generic ranges in relation to the British Dinantian stages but failed to repeat the Eoparastaffella record of George et al. (1976) in the Chadian stratotype at Chatburn. The absence of this guide fossil in the Chatburn section and the Chadian biostratigraphy of the Craven Basin were discussed in detail by Riley (1990, 1993, 1995) who stressed that the correlation of the base of the Chadian is impossible and that it is only the late Chadian that has a distinct biostratigraphic signature. A review of the validity of the Chadian and of the suitability of the Chatburn section as its stratotype is provided in Adams et al. (2004).

In the last decade the search for a new T-V boundary stratotype in Eurasia has contributed to a substantial improvement in biostratigraphic resolution in this early Carboniferous interval (Devuyst 2006, Devuyst \& Kalvoda 2007, Hance et al. 2011, Kalvoda et al. 2010, Poty et al. 2006). In the zonation of Devuyst \& Hance (in Poty et al. 2006) the latest Tournaisian foraminiferal zone MFZ8 is defined by the entry of Eoparastaffella and Biseriella bristolensis (Reichel). That is, it is coincident with the 


\section{Three Cliffs Bay}

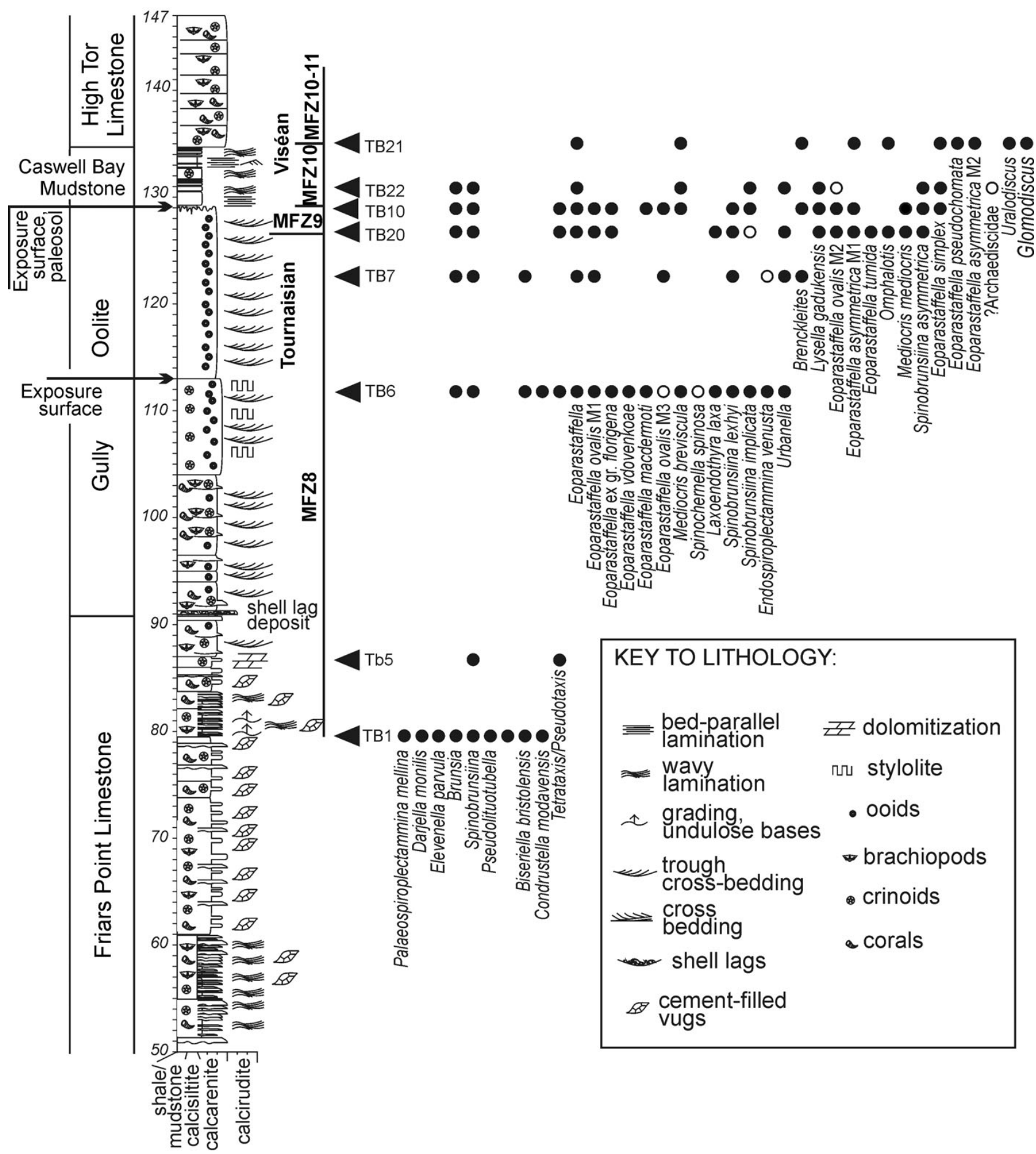

Figure 7. Distribution of most important foraminifers in the Three Cliffs Bay section in South Wales-Mendip Shelf. Foraminiferal zones according to Devuyst \& Hance in Poty et al. (2006). White circles mean $c f$. identifications. MFZ10 in Caswell Bay Mudstone is based on the correlation with the Ogmore section.

original definition of the Chadian base. The base of the first Viséan zone MFZ 9 coincides with the FAD of Eoparastaffella simplex Vdovenko in an evolutionary lin- eage from Eoparastaffella ovalis Vdovenko (Devuyst et al. 2003, Work 2008). The detailed biostratigraphy of the T-V boundary interval (Fig. 3) is characterized by successive 
Figure 8. Distribution of most important foraminifers in the $\mathrm{Og}$ more section in the Vale of Glamorgan in South Wales-Mendip Shelf. Foraminiferal zones according to Devuyst \& Hance in Poty et al. (2006).

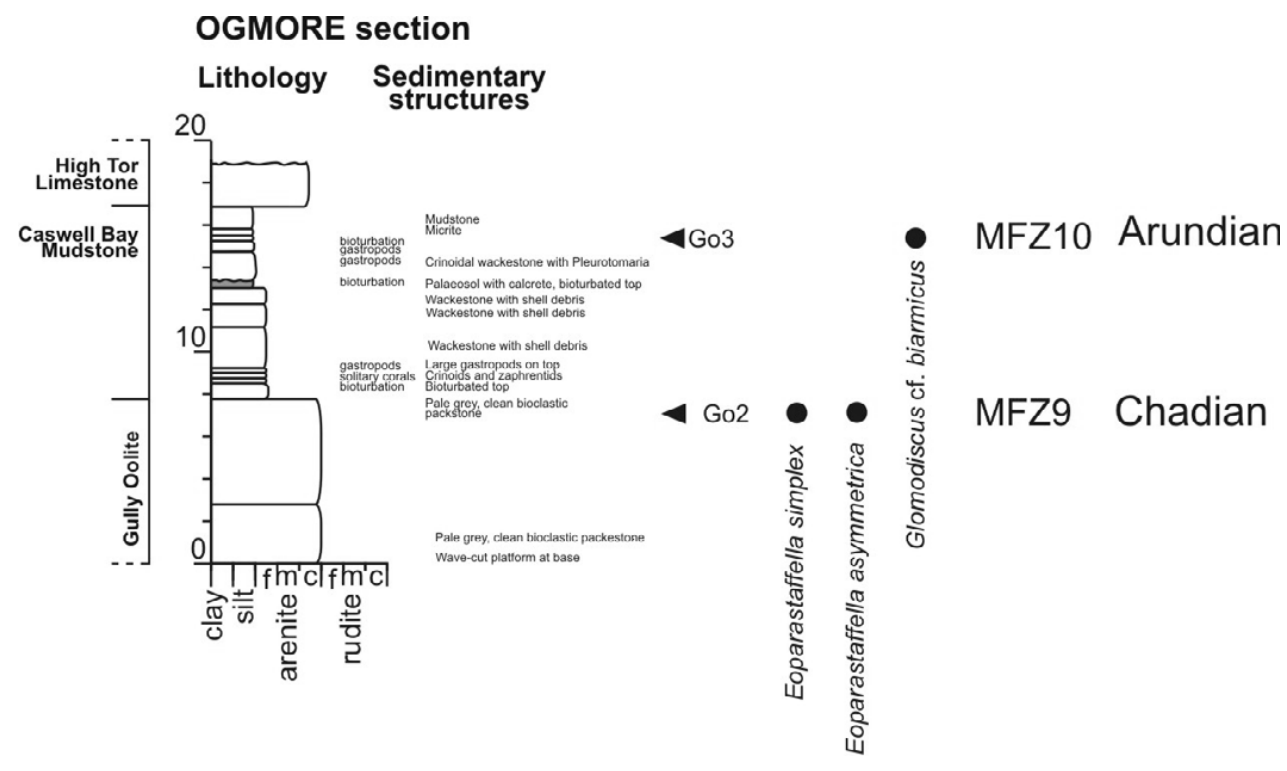

FADs of Eoparastaffella vdovenkoae Devuyst \& Kalvoda, Lysella gadukensis Bozorgnia, Eoparastaffella ex gr. florigena (Pronina), Eoparastaffella ovalis M3 and Eoparastaffella ovalis M2 below the T-V boundary and Eoparastaffella asymmetrica Vdovenko and Eoparastaffella tummida (Pronina) just above the boundary (Devuyst 2006, Devuyst \& Kalvoda 2007; Fig. 3). The entry of Eoparastaffella ovalis M2 is very close to the entry of E. simplex and because in many sections E. ovalis $\mathrm{M} 2$ is more common than E. simplex, it can be used as an alternative criterion to identify the T-V boundary (Devuyst 2006; Kalvoda et al. 2010, 2011). In accord with the base of the Arundian, the base of MFZ10 coincides with the entry of Archaediscidae represented by Ammarchaediscus and Planoarchaediscus, while the evolutionary younger Uralodiscus and Glomodicus occur in the upper part of the zone (Devuyst \& Hance in Poty et al. 2006).

\section{North Staffordshire}

\section{Ladyside Wood}

Figures 10-19

At Ladyside Wood the top of the Waulsortian facies is characterised by the dominance of skeletal debris of crinoids, ostracods, brachiopods and bryozoans. The multichambered foraminifers present included mostly only small juvenile Endothyra, Spinobrunsiina and Brunsia. These are accompanied by rare Eblanaia michoti (Conil \& Lys) and ?Eoparastaffella (Fig. 4). The presence of ?Eoparastaffella may indicate latest Tournaisian age (MFZ8). Unlike at Brown End Quarry, Tetrataxis and Eotextularia diversa (Chernysheva) are absent while Eblanaia is present. The richest foraminiferal association in the lowermost part of the section was obtained in sample M4. In addition to previous taxa, Bessiella cf. agilis Conil, Bessiella mobilis (Conil \& Lys), Darjella monilis Malakhova, Plectogyranopsis regularis (Rauzer-Chernousova), Pseudolituotubella and Omphalotis occur, the association being characteristic of MFZ8. On the other hand a guide of MFZ4 in Belgium Spinochernella brencklei Conil \& Lys, occurs and continues to the lowermost Viséan. This bed is followed by an interval up to the level of sample LW14 where micritic facies dominate with small juveniles multichambered foraminifers represented mostly by Endothyra, Spinobrunsiina and Brunsia. The very rare presence of Eoparastaffella supports the latest Tournaisian age of MFZ8.

The end of the Tournaisian is marked by bioclastic limestones with a very rich foraminiferal association in which Bessiella, Spinobrunsiina, Spinochernella and Eblanaia are abundant. This and the absence or very rare occurrence of Florennella show very good fit with the Chadian stratotype in Chatburn (Riley 1995), Irish sections in Oughterard, Lane and Rush (Devuyst 2006, Kalvoda et al. 2011) and sections in the Namur Basin (Hance et al. 1981, Hance 1988). There is also some faunistic similarity with the North American Cordillera (Brenckle 1973, Mamet et al. 1986, Skipp 1969). The presence of Bessiella legrandi Conil \& Hance, Eosinopsis sp. 2 Vachard \& Hance, Carbotarima or Forschiella may indicate already the upper part of the MFZ8 but the important Eoparastaffella guides are missing. Forschiella- once regarded as an early Viséan guide (Rauser-Chernoussova et al. 1996) has been recently reported from the latest Tournaisian of the Dublin Basin (Kalvoda et al. 2011). Carbotarima was originally described by Brenckle (2004) from early late Viséan beds along the northern margin of the Tarim Basin. Recently Hance et al. (2011) reported the genus from the 
late Tournaisian to middle Viséan of south China. The occurrences of Eosinopsis are typical for the latest Tournaisian and earliest Viséan MFZ8 and MFZ9 in China, the Urals and Western Europe; Eosinopsis sp. 2 being reported in the MFZ8 (Hance et al. 2011). Among abundant Bessiella the variable group B. rectiformis (Bogush \& Yuferev) dominates (Groessens et al. 1982, Hance 1988).

A distinctly different foraminiferal association is recognised in sample Wo3 in which Eoparastaffella and Lysella are abundant and Paralysella is also present. Lysella gadukensis becomes an important component of tuberculate foraminiferal association at this level. Bessiella is still abundant dominated by the B. rectiformis group and Eblanaia is still present. Eblanaia and Eoparastaffella usually do not co-occur, the sample thus probably records the transportation of foraminifers from different environments. Among Eoparastaffella, members of the Eoparastaffella florigena group are especially common while members of the Eoparastaffella ovalis-simplex group are virtually absent. Nevertheless, the presence of Eoparastaffella tummida (Pronina) indicates already a Viséan age of the MFZ9.

Above, an interval of biomicritic limestones follows (see Fig. 4). The sample Wo96 shows a complete return to a foraminifera association, which consists exclusively of taxa known from the Tournaisian, dominated by Spinobrunsiina, Bessiella, Eblanaia, Brunsia, while Eoparastaffella is completely absent here. An interval of biomicritic limestones follows with low foraminiferal representation in which again small juveniles dominate (Spinobrunsiina, Brunsia, Endothyra) similarly as in some late Tournaisian facies.

In the first bioclastic limestone (sample Wo6) a return to the trend initiated in the first Viséan sample Wo3 is apparent. The Eoparastaffella association is abundant but more diversified than in Wo3. This association is dominanted by members of the E. florigena group, but E. ovalis M2 is also abundant. E. simplex is however very rare - only one specimen being recorded from close to one hundred Eoparastaffella axial sections. E. asymmetrica represents another important Viséan guide. Among the tuberculate foraminifers Lysella gadukensis becomes a dominant component while Spinobrunsiina is still abundant and Bessiella becomes rare. Characteristic deviations in the last coil of Eoparastaffella, recorded also in some of the samples above (including Wo3), and in samples described by Devuyst from Irish sections (Devuyst 2006), are typical. These are most likely to have been caused by environmental factors and are of no stratigraphic significance.

A similarly rich foraminifer association is present in Wo7. Here representatives of Eoparastaffella are diverse and abundant once again with members of the E. florigena group dominating the association. E. ovalis and group E. interiecta Vdovenko group are also present. Viséan guides are represented by very rare E. asymmetrica and E. simplex (1 specimen in more than 100 axial sections) but $E$. ovalis M2 and M3 are both common. Among the tuberculate foraminifers Spinobrunsiina, Bessiella and the L. gadukensis group are dominant. Brunsia is abundant and Eblanaia still present. The richness of Spinobrunsiina, abundant Brunsia and Bessiella is similar to that seen in sections of equivalent age in the Namur Basin (Hance 1979, Hance et al. 1981). The common occurrence of both Eoparastaffella and the aforementioned association of tuberculate foraminifers within calciturbidites indicates that they were derived from different source areas.

Wo9 is dominated by smaller foraminifers often represented by juveniles. Spinobrunsiina and Brunsia are abundant. Despite the granulometry being suitable for E. simplex, the assemblage is dominated by juvenile members of E. florigena or E. interiecta group, indicating a derivation from those parts of the ramp where E. simplex was absent. Evidently the niches of Eoparastaffella species were varied.

A diverse association of dainellids including Lysella, Bessiella and Florennella is present again in Wo10 where juveniles are especially abundant. Spinobrunsiina is not as abundant as in previous cases. Among the Eoparastaffella, members of the variable E. florigena group continue to dominate. Stratigraphically important is the FAD of Eoparastaffella restricta Postoyalko. Eosinopsis resembling E. solida Vachard \& Hance enters. Similar trends can be seen also in Wo11, monotonous Eoparastaffella is represented only by the E. florigena group.

In Wo19 dainellids are represented mainly by the L. gadukensis group while Bessiella is absent and Spinobrunsiina is more abundant. In Eoparastaffella the dominance of the variable E. florigena group continues as it does in Wo22 where also rare occurrence of E. asymmetrica and E. cf. simplex are recorded. A diverse association of dainellids is present once again dominated by L. gadukensis. Bessiella and Florennella are also present. In the last studied sample Wo28 the dominance of Lysella ex gr. gadukensis and E. florigena group continues, while the entry of Plectogyranopsis convexus (Rauser-Chernousova) is stratigraphically important.

\section{Brown End}

Figures 24-28

At the base of the measured section (B38-B40) in Brown End Quarry is a peri-Waulsortian facies charactersised by crinoids, ostracods, bryozoans and salebrids. Foraminifers are also present including Eotextularia diversa and Tetrataxis (Lees 1997).

The interval above (B37-B12) is dominated by micritic limestones (mostly wackestones) with abundant sponge 
Jiří Kalvoda et al. • High resolution biostratigraphy of the Tournaisian-Viséan boundary interval in the North Staffordshire Basin

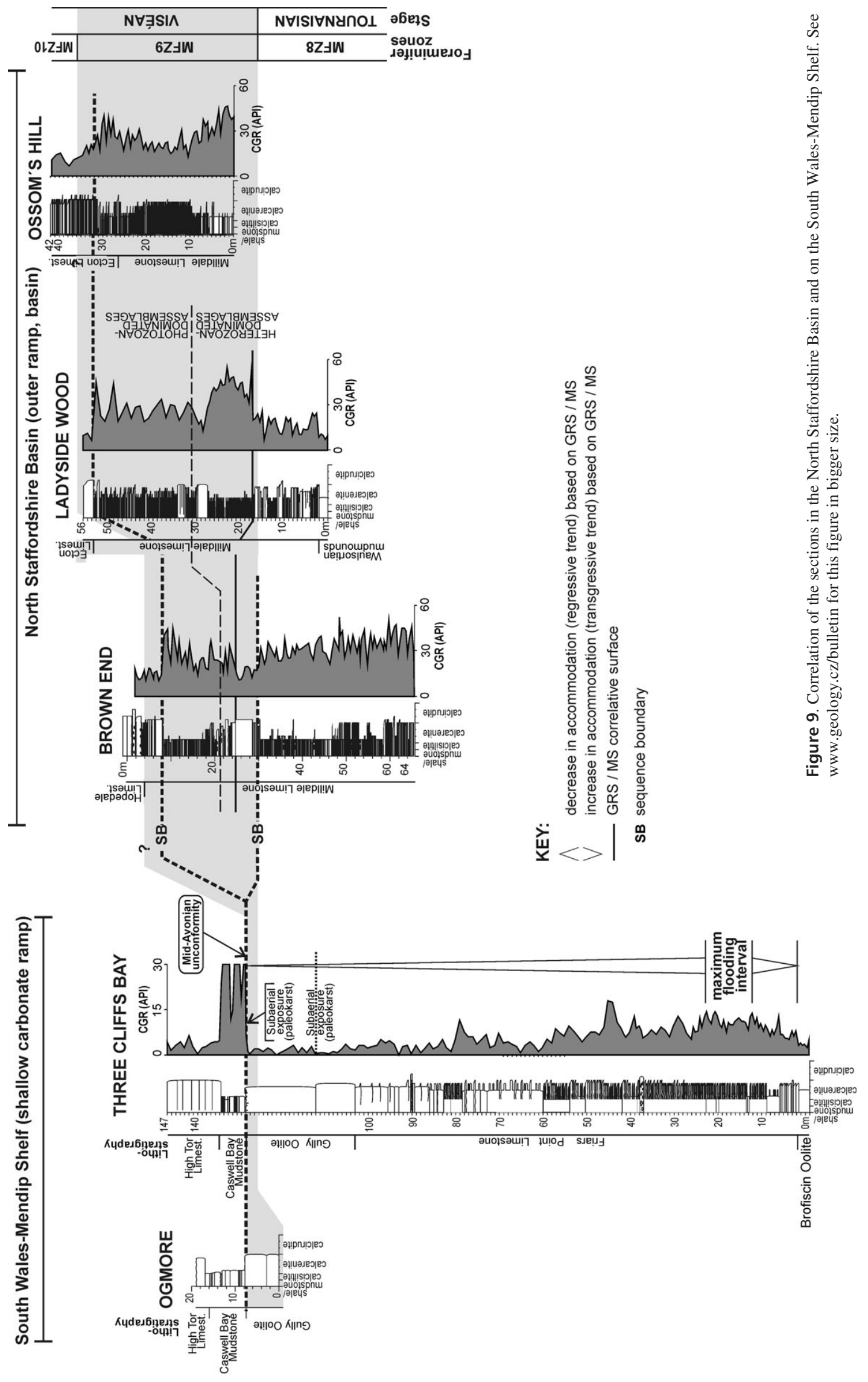


Figure 10. Typical foraminifers from Ladyside Wood in the North Staffordshire Basin. • A - Spinochernella sp., Ladyside Wood, LW5, t.s. 5. - B - Spinochernella sp., Ladyside Wood, Wo1, t.s. 27. • C - Spinochernella spinosa (Chernysheva), Ladyside Wood, Wo2, t.s. 4. • D - Spinochernella spinosa (Chernysheva), Ladyside Wood, Wo1, t.s. 2.・E - Spinochernella spinosa (Chernysheva), Ladyside Wood M4, t.s. 1. • F - Spinochernella paraukrainica (Lipina), Ladyside Wood, Wo2, t.s. 17. $\bullet \mathrm{G}$ - Spinochernella spinosa (Chernysheva), Ladyside Wood, LW15, t.s. 4 . $・ H$ - Spinochernella spinosa (Chernysheva), Ladyside Wood, Wo2, t.s. 12. I - Spinochernella spinosa (Chernysheva), Ladyside Wood, Wo2, t.s. 7. • J - Spinochernella sp., Ladyside Wood, Wo1, t.s. 8. • K - Spinobrunsiina vachardi Conil, Wo2, t.s. 1. • L - Spinobrunsiina pietoni (Conil \& Lys), Ladyside Wood, M4, t.s. 5. - M - Spinobrunsiina landeliesi Conil, Ladyside Wood, Wo6, t.s. 7. • N - Spinobrunsiina vachardi Conil, Ladyside Wood, Wo28, t.s. 1. - O - Spinobrunsiina lidijae (Brazhnikova), Ladyside Wood, LW6, t.s. 3. • P - Spinochernella cf. spinosa (Chernysheva), Ladyside Wood, Wo1, t.s. 18. - Q - Spinochernella brencklei Conil \& Lys, Ladyside Wood, LW15, t.s. 1. • R - Spinochernella brencklei Conil \& Lys, Ladyside Wood, Wo1, t.s. 3. - S - Spinochernella brencklei Conil \& Lys, Ladyside Wood, Wo3, t.s. 14. Enlargement: 75×, L 100×.

Figure 11. Typical foraminifers from Ladyside Wood in the North Staffordshire Basin. A plate showing the variability of the Spinobrunsiina lexhyi group. • A - Spinobrunsiina cf. lexhyi Conil \& Hance, Ladyside Wood, LW15, t.s. 1. • B - Spinobrunsiina lexhyi Conil \& Hance, Ladyside Wood, Wo1, t.s. 24. C - Spinobrunsiina cf. lexhyi Conil \& Hance, Ladyside Wood, Wo11, t.s. 2. • D - Spinobrunsiina lexhyi Conil \& Hance, Ladyside Wood, Wo2, t.s. 13. E-Spinobrunsiina lexhyi Conil \& Hance, Ladyside Wood, Wo1, t.s. 3. • F - Spinobrunsiina lexhyi Conil \& Hance, Ladyside Wood, Wo2, t.s. 18. - G - Spinobrunsiina lexhyi Conil \& Hance, Ladyside Wood, Wo22, t.s. 6. • H - Spinobrunsiina lexhyi Conil \& Hance, Ladyside Wood, Wo7, t.s. 54. - I - Spinobrunsiina lexhyi Conil \& Hance, Ladyside Wood, Wo22, t.s. 3. • J - Spinobrunsiina cf. lexhyi Conil \& Hance, Ladyside Wood, Wo1, t.s. 2. - K - Spinobrunsiina cf. lexhyi Conil \& Hance, Ladyside Wood, LW16, t.s. 7. • L - Spinobrunsiina lexhyi Conil \& Hance, Ladyside Wood, M4, t.s. 2. - M - Spinobrunsiina lexhyi Conil \& Hance, Ladyside Wood, M4, t.s. 5. • N - Spinobrunsiina cf. lexhyi Conil \& Hance, Ladyside Wood, Wo2, t.s. 11. - O - Spinobrunsiina lexhyi Conil \& Hance, Ladyside Wood, LW5, t.s. 5. • P - Spinobrunsiina lexhyi Conil \& Hance, Ladyside Wood, Wo28, t.s. 1. - Q - Spinobrunsiina lexhyi Conil \& Hance, Ladyside Wood, Wo7, t.s. 16. • R - Spinobrunsiina lexhyi Conil \& Hance, Ladyside Wood, Wo19, t.s. 3. -S - Spinobrunsiina cf. lexhyi Conil \& Hance, Ladyside Wood, M4, t.s. 5. • T - Spinobrunsiina sp., Ladyside Wood, Wo2, t.s. 16. $\bullet$ U - Spinobrunsiina imlicata (Conil \& Lys), Ladyside Wood, Wo19, t.s. 1.・V - Spinobrunsiina imlicata (Conil \& Lys), Ladyside Wood, Wo6, t.s. 4. • W - Spinobrunsiina cf. imlicata (Conil \& Lys), Ladyside Wood, LW6, t.s. 2. Enlargement: 75×, U and W 100×.

Figure 12. Typical foraminifers from Ladyside Wood in the North Staffordshire Basin. • A - Carbotarima sp., Ladyside Wood, LW16, t.s. 3. -B - Carbotarima sp., Ladyside Wood, Wo1, t.s. 8. C - Carbotarima sp., Ladyside Wood, Wo2, t.s. 10. •D - ?Carbotarima sp., Ladyside Wood, LW15, t.s. 1. E - Eosinopsis sp. 2 Vachard \& Hance, Ladyside Wood, LW16, t.s. 7. • F - Eosinopsis sp. 2 Vachard \& Hance, Ladyside Wood, Wo1, t.s. 25. - G - Spinobrunsiina ramsbottomi Conil \& Longerstaey, Ladyside Wood, Wo28, t.s. 2. $\bullet$ H - Eosinopsis primaeva Vachard \& Hance, Ladyside Wood, Wo1, t.s. 7. $・$ I - Eosinopsis sp. 2 Vachard \& Hance, Ladyside Wood, LW16, t.s. 4. $・$ J - Eosinopsis primaeva Vachard \& Hance, Ladyside Wood, Wo1, t.s. 25. $・ \mathrm{~K}-$ Eosinopsis sp., Ladyside Wood, Wo22, t.s. 4. $・$ L - Plectogyranopsis convexa (Rauser-Chernousova), Ladyside Wood, Wo28, t.s. 2. $\bullet$ M - Eosinopsis sp. 2 Vachard \& Hance, Ladyside Wood, Wo1, t.s. 6. $\bullet$ - Plectogyranopsis regularis (Rauser-Chernousova), Ladyside Wood, M4, t.s. 1. Enlargement: $75 \times, \mathrm{N} 100 \times$.

Figure 13. Typical foraminifers from Ladyside Wood in the North Staffordshire Basin. • A - Plectogyranopsis sp., Ladyside Wood, Wo28, t.s. 6. - B - Plectogyranopsis paraconvexus (Brazhnikova \& Rostovtseva), Ladyside Wood, Wo7, t.s. 28. • C - Eosinopsis sp., Ladyside Wood, Wo6, t.s. 10. -D - Forschiella sp., Ladyside Wood, Wo6, t.s. 21. $・$ E - Condrustella modavensis (Conil \& Lys), Ladyside Wood, Wo3, t.s. 28. $\bullet$ F - Conilites dinantii (Conil \& Lys), Ladyside Wood, Wo1, t.s. $6 \bullet \mathrm{G}-$ Conilites dinantii (Conil \& Lys), Ladyside Wood, Wo3, t.s. $32 \cdot \bullet \mathrm{H}-$ Conilites dinantii (Conil \& Lys), Ladyside Wood, Wo3, t.s. 29.・I - Conilites dinantii (Conil \& Lys), Ladyside Wood, Wo1, t.s. 19.・ J - Eblanaia michoti (Conil \& Lys), Ladyside Wood, Wo10, t.s. 13. $• \mathrm{~K}-$ Eblanaia michoti (Conil \& Lys), Ladyside Wood, LW16, t.s. 6. $\bullet$ L - Eblanaia michoti (Conil \& Lys), Ladyside Wood, Wo1, t.s. 27. - M - Eblanaia michoti (Conil \& Lys), Ladyside Wood, Wo1, t.s. 17. $\mathrm{N}$ - Valvulinella ceunacumensis Hance, Ladyside Wood, Wo3, t.s. 30. Enlargement: A-C, E, H-I 75x; D, F-G, J-N 50x.

Figure 14. Typical foraminifers from Ladyside Wood in the North Staffordshire Basin. $\bullet$ A - Bessiella rectiformis (Bogush \& Yuferev), Ladyside Wood, Wo1, t.s. 11. B - Bessiella rectiformis (Bogush \& Yuferev), Ladyside Wood, Wo1, t.s. 25. $・$ C - Bessiella rectiformis (Bogush \& Yuferev), Ladyside Wood, Wo2, t.s. 10. D - Bessiella rectiformis (Bogush \& Yuferev), Ladyside Wood, Wo2, t.s. 6. $\bullet$ E - Bessiella rectiformis (Bogush \& Yuferev), Ladyside Wood, Wo2, t.s. 5. $• \mathrm{~F}$ - Bessiella rectiformis (Bogush \& Yuferev), Ladyside Wood, Wo7, t.s. 15. $\bullet \mathrm{G}-$ Bessiella leusoniae Conil, Ladyside Wood, M4, t.s. 5. $\bullet \mathrm{H}-$ Mediocris mediocris (Vissarionova), Ladyside Wood, Wo9, t.s. 6. $\bullet$ I - Bessiella cf. agilis Conil, Ladyside Wood, Wo1, t.s. 9. • J - Bessiella leusoniae Conil, Ladyside Wood, Wo3, t.s. 12. $・ \mathrm{~K}-$ Bessiella rectiformis (Bogush \& Yuferev), Ladyside Wood, Wo2, t.s. 6. $\cdot \mathrm{L}-$ Mediocris mediocris (Vissarionova), Ladyside Wood, Wo3, t.s. 12. $\bullet \mathrm{M}-$ Bessiella agilis Conil, Ladyside Wood, Wo2, t.s. 7. $\bullet \mathrm{N}-$ Bessiella leusoniae Conil, Ladyside Wood, Wo1, t.s. 9. $\bullet \mathrm{O}-$ Bessiella agilis Conil, Ladyside Wood, Wo2, t.s. 5. $\bullet$ P - Bessiella legrandi Conil \& Hance, Ladyside Wood, Wo1, t.s. 9. $\bullet$ Q - Bessiella leusoniae Conil, Ladyside Wood, Wo1, t.s. 23. $・$ R - Bessiella legrandi Conil \& Hance, Ladyside Wood, Wo2, t.s. 17. Enlargement: $75 \times, \mathrm{H}$ and L 100x. 

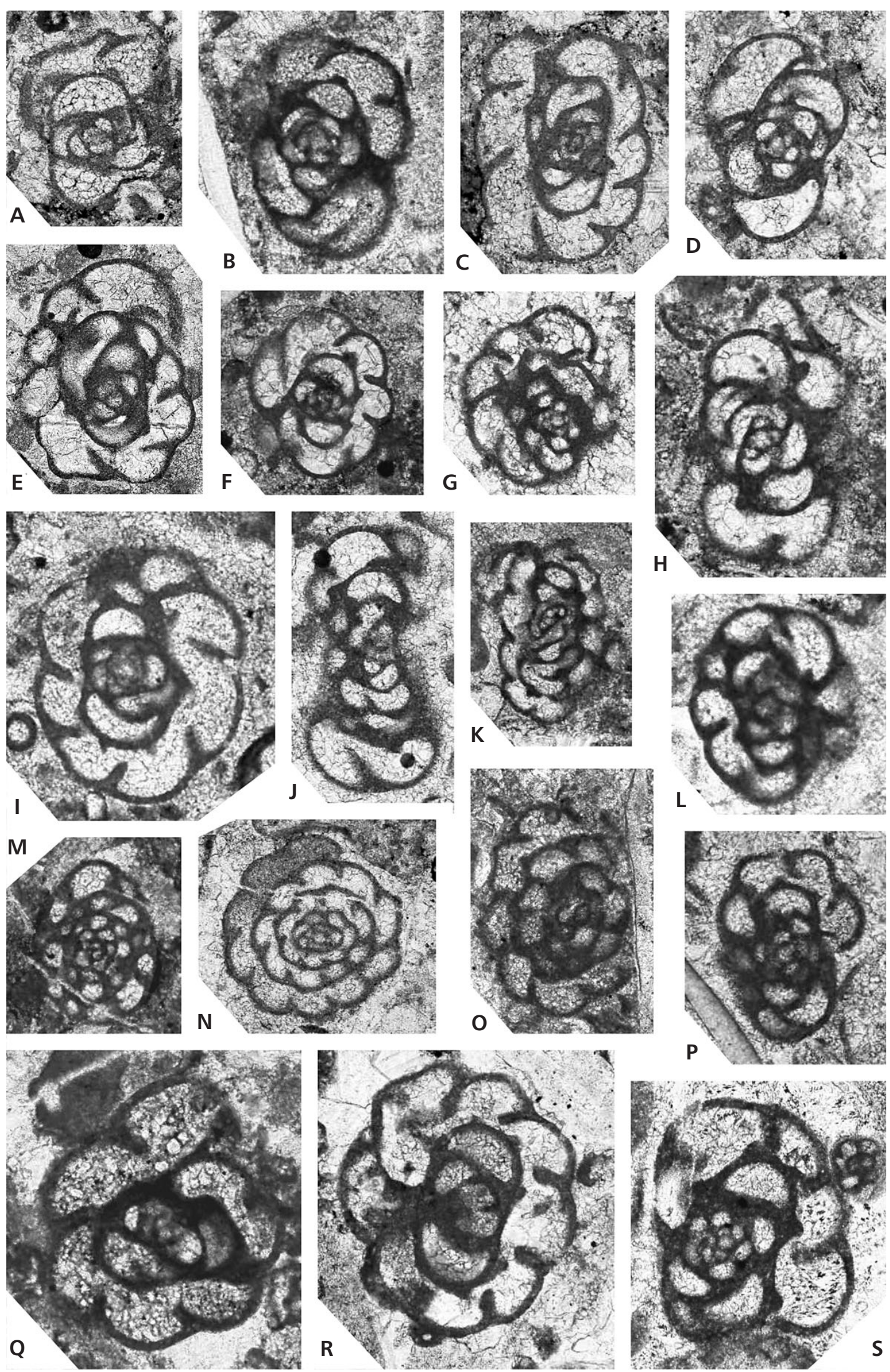

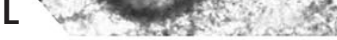
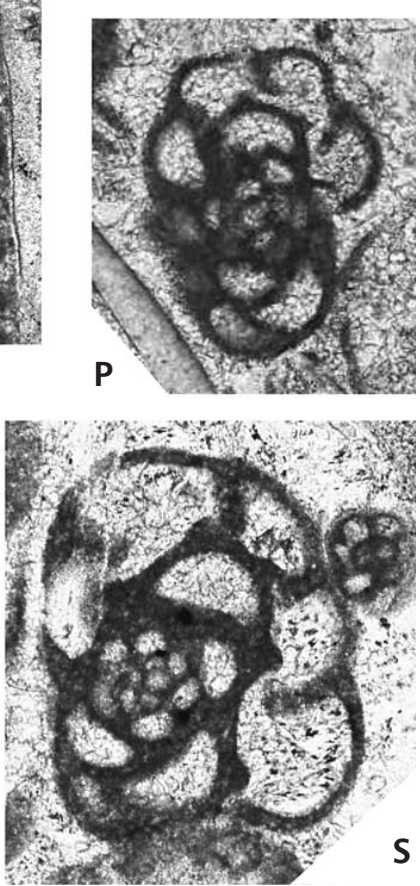

Figure 10. Typical foraminifers from Ladyside Wood in the North Staffordshire Basin. For explanation see page 510. 

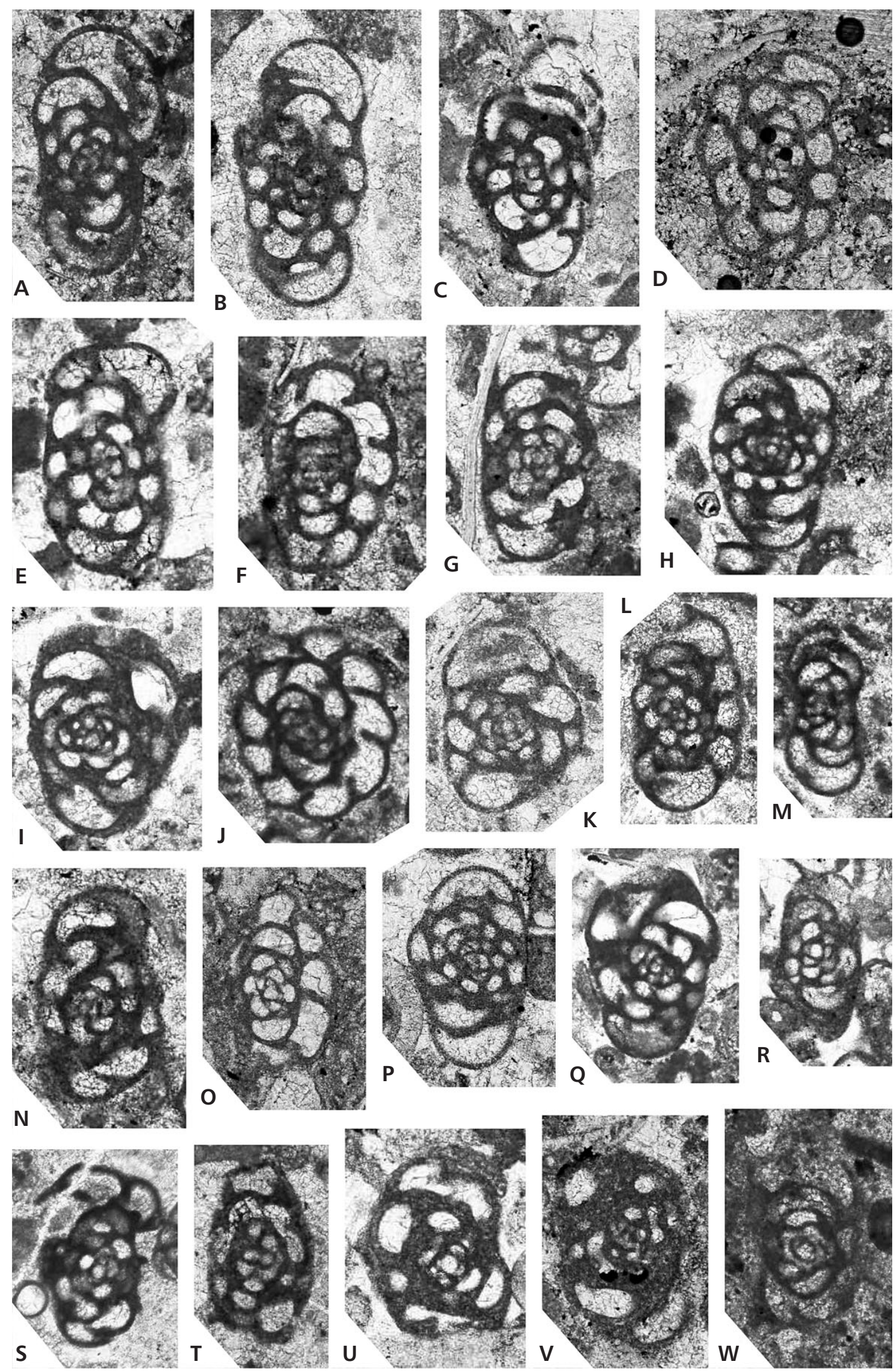

Figure 11. Typical foraminifers from Ladyside Wood in the North Staffordshire Basin. A plate showing the variability of the Spinobrunsiina lexhyi group. For explanation see page 510. 


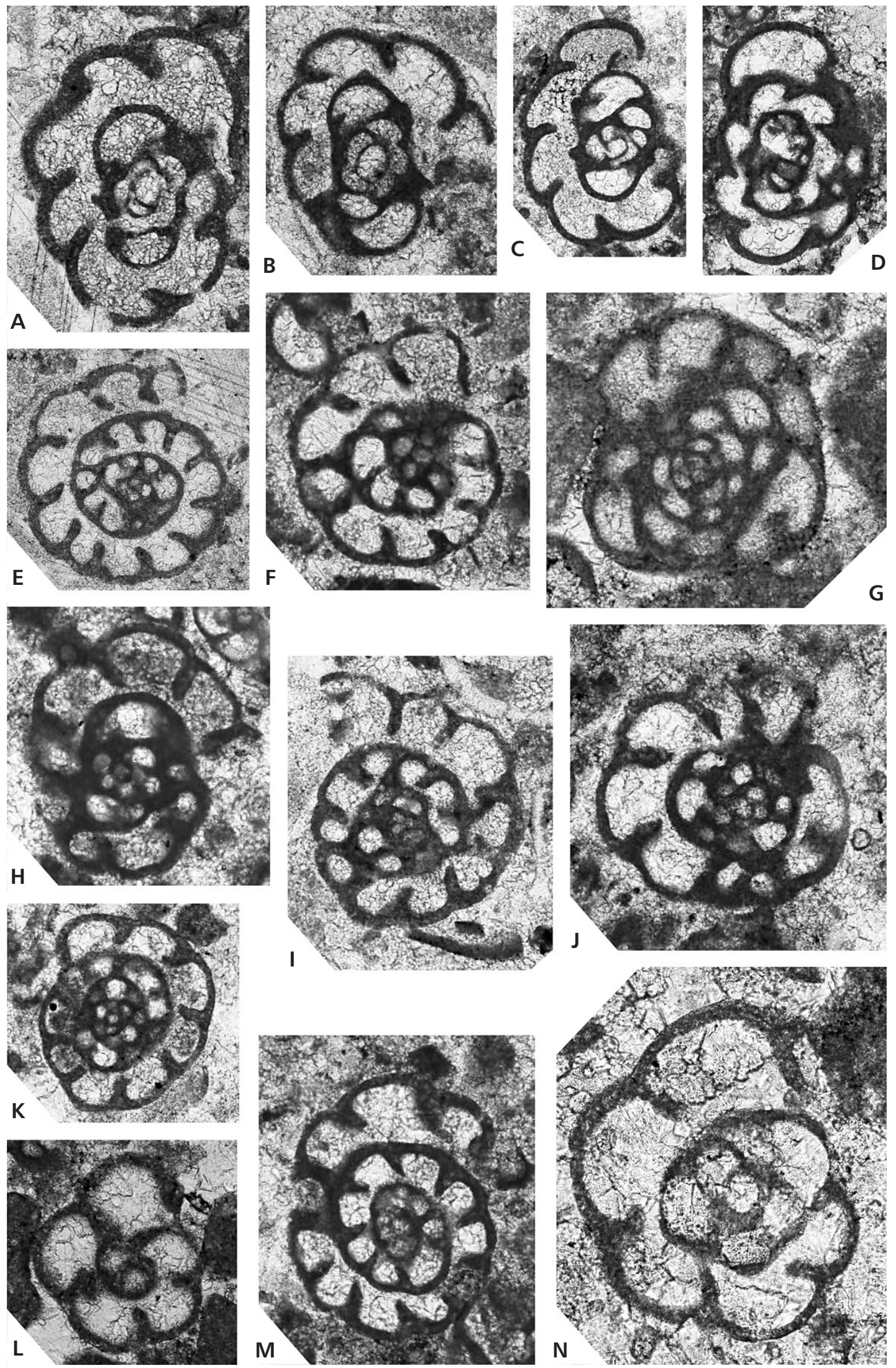

Figure 12. Typical foraminifers from Ladyside Wood in the North Staffordshire Basin. For explanation see page 510. 

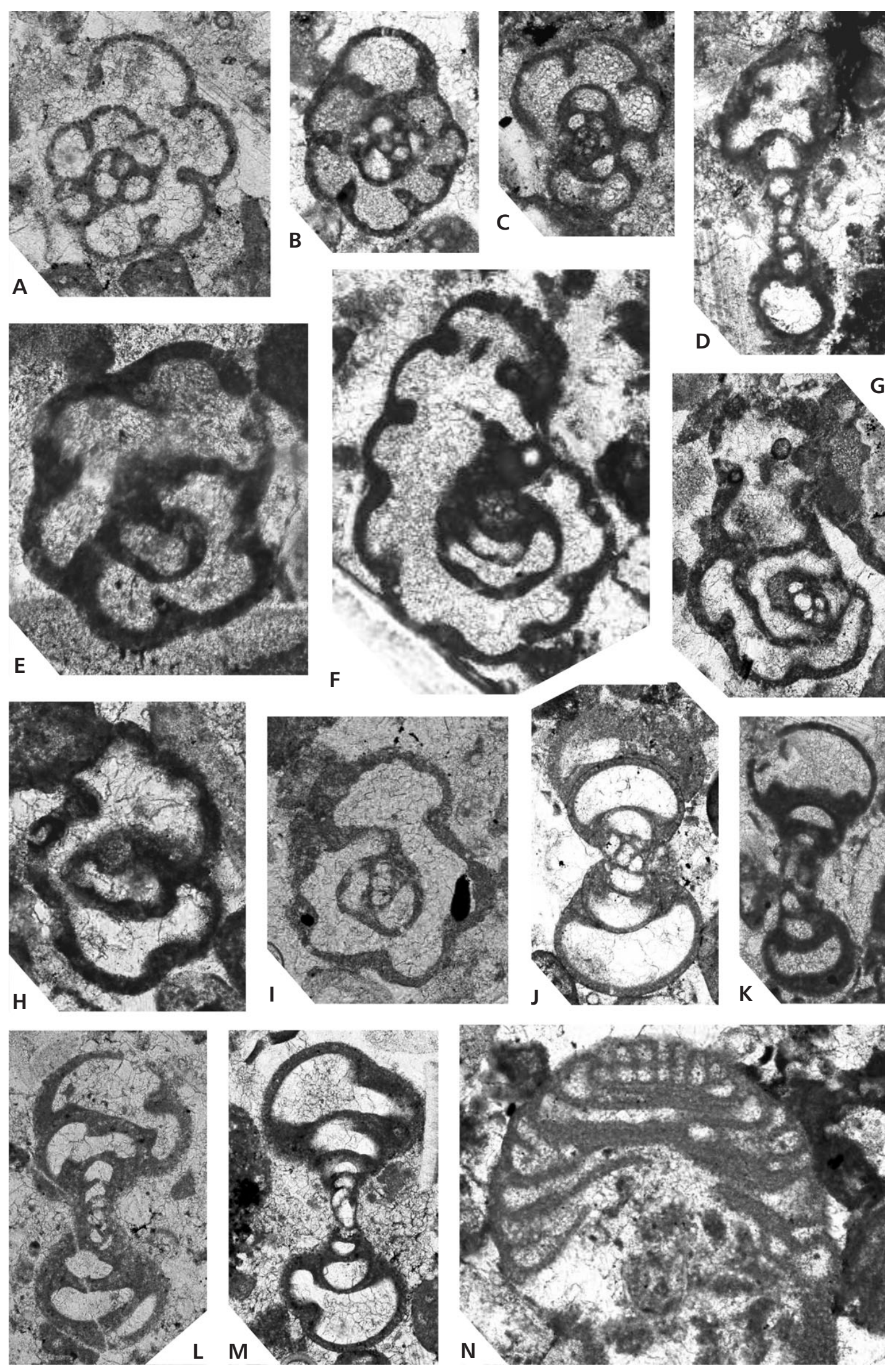

Figure 13. Typical foraminifers from Ladyside Wood in the North Staffordshire Basin. For explanation see page 510. 

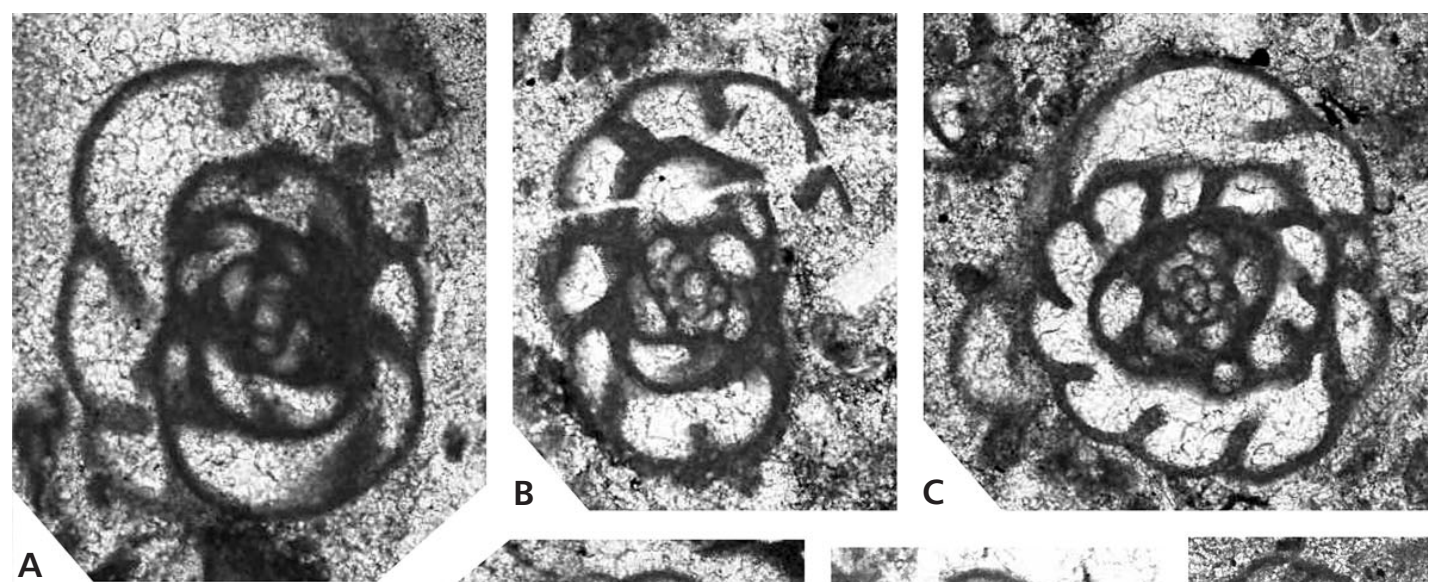
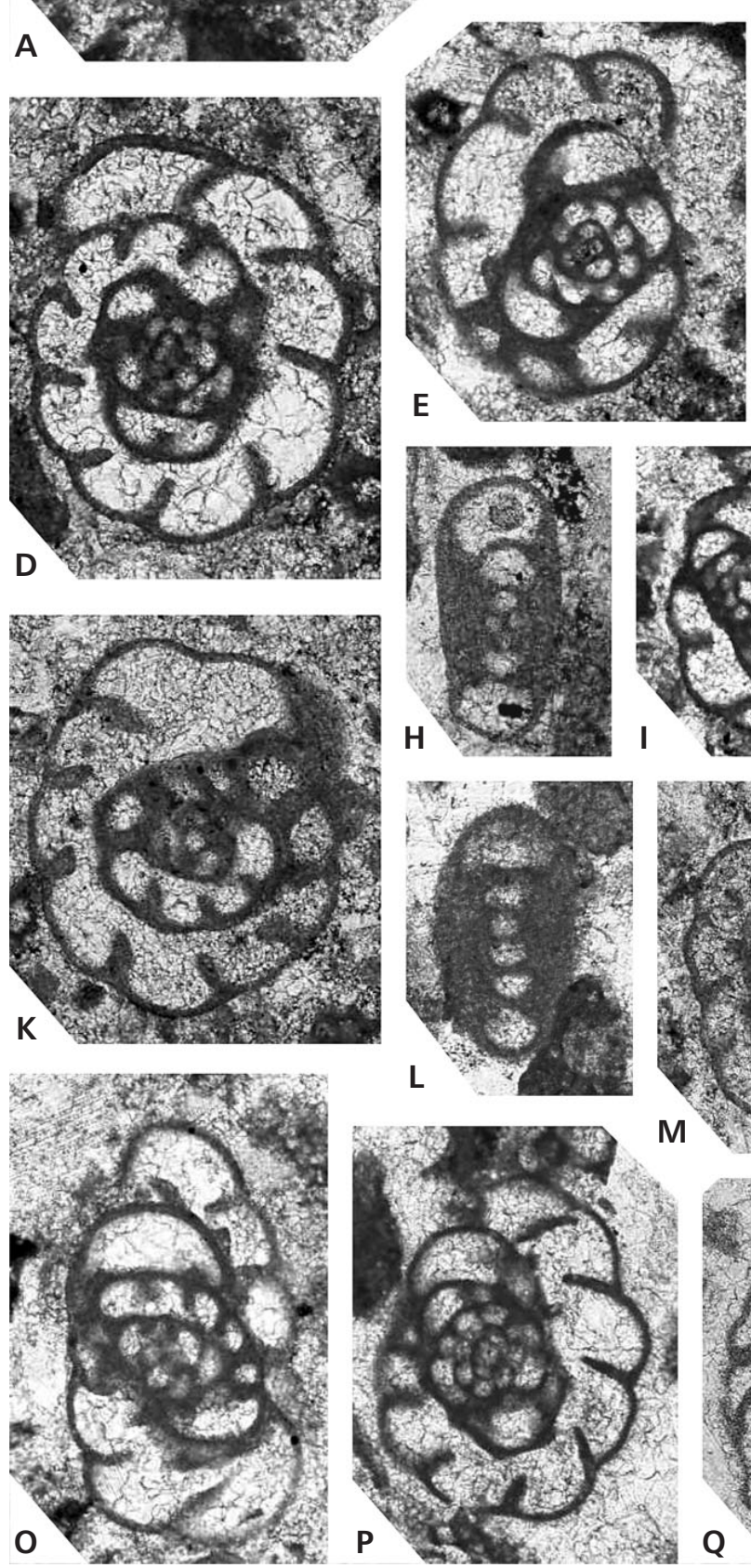
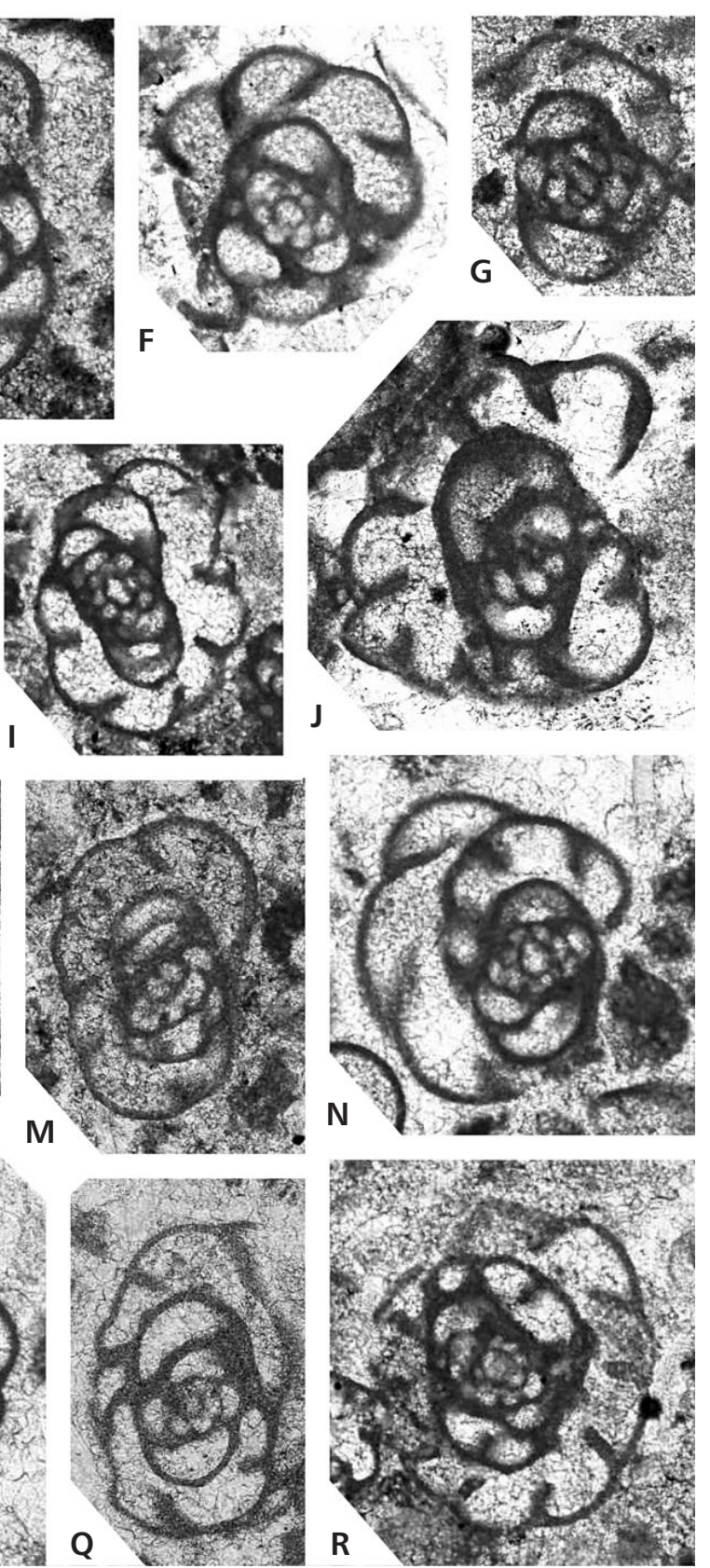

Figure 14. Typical foraminifers from Ladyside Wood in the North Staffordshire Basin. For explanation see page 510. 
spicules and ostracods. Other than Earlandia, foraminifers are quite rare, these being represented by small juveniles which were difficult to identify. Foraminifers that were identified include Endothyra, Spinobrunsiina, Brunsia and the stratigraphically important Elevenella parvula which together suggest an uppermost MFZ7-MFZ8 age.

Figure 15. Typical foraminifers from Ladyside Wood in the North Staffordshire Basin. • A - Florennella stricta (Conil \& Lys), Ladyside Wood, LW8, t.s. 4. $\bullet$ B - Florennella stricta (Conil \& Lys), Ladyside Wood, LW15, t.s. 5. $\bullet$ C - Florennella stricta (Conil \& Lys), Ladyside Wood, Wo1, t.s. 12. $\cdot$ D - Bessiella mobilis (Conil \& Lys), Ladyside Wood, M4, t.s. 5. E - Florennella stricta (Conil \& Lys), Ladyside Wood, Wo1, t.s. 24. $\bullet$ F - Florennella stricta (Conil \& Lys), Ladyside Wood, Wo1, t.s. 21. $\bullet \mathrm{G}-$ Florennella cf. amplissima Conil, Ladyside Wood, LW15, t.s. 1. $\bullet \mathrm{H}-$ Florennella stricta (Conil \& Lys), Ladyside Wood, Wo2, t.s. 12.・I - Lysella gadukensis Bozorgnia, Ladyside Wood, Wo9, t.s. 10. Ladyside Wood, Wo10, t.s. 7. $\bullet \mathrm{K}-$ Florennella stricta (Conil \& Lys), Ladyside Wood, LW16, t.s. 3. $\bullet$ L - Florennella stricta (Conil \& Lys), Ladyside Wood, Wo2, t.s. 12. $\bullet$ M - Lysella gadukensis Bozorgnia, Ladyside Wood, Wo3, t.s. 31. $・ \mathrm{~N}$ - Lysella gadukensis Bozorgnia, Ladyside Wood, Wo19, t.s. 4. $\bullet \mathrm{O}-$ Lysella gadukensis Bozorgnia, Ladyside Wood, Wo3, t.s. 39. Enlargement: $75 \times$.

Figure 16. Typical foraminifers from Ladyside Wood in the North Staffordshire Basin. • A - Lysella gadukensis Bozorgnia, Ladyside Wood, Wo3, t.s. 26. B - Lysella gadukensis Bozorgnia, Ladyside Wood, Wo22, t.s. 6. $\bullet$ C - Paralysella sp., Ladyside Wood, Wo3, t.s. 7. • D - Paralysella sp., Ladyside Wood, Wo3, t.s. 7. $\bullet$ E - Paralysella sp., Ladyside Wood, Wo28, t.s. 5. $・$ F - ?Paralysella sp., Ladyside Wood, Wo3, t.s. $44 . \bullet \mathrm{G}-$ Paralysella sp., Ladyside Wood, Wo3, t.s. 49. $・$ H - Brenckleites sp., Ladyside Wood, Wo3, t.s. 49. • I - Paralysella cf. procerula (Malakhova), Ladyside Wood, Wo6, t.s. 3.・J - Paralysella sp., Ladyside Wood, Wo7, t.s. 22. $\bullet \mathrm{K}-$ Urbanella urbana (Malakhova), Ladyside Wood, Wo6, t.s. 1. $\bullet$ L - Urbanella urbana (Malakhova), Ladyside Wood, Wo6, t.s. 28. • M - Urbanella urbana (Malakhova), Ladyside Wood, Wo28, t.s. 5. $・$ N - Eoparastaffella macdermoti Devuyst \& Kalvoda, Ladyside Wood, Wo7, t.s. 16. - O - Eoparastaffella vdovenkoae Devuyst \& Kalvoda, Ladyside Wood, Wo7, t.s. 39. - P - Eoparastaffella vdovenkoae Devuyst \& Kalvoda, Ladyside Wood, Wo6, t.s. 16. $\bullet$ Q - Eoparastaffella vdovenkoae Devuyst \& Kalvoda, Ladyside Wood, Wo22, t.s. 1. $・$ R - Eoparastaffella macdermoti Devuyst \& Kalvoda, Ladyside Wood, Wo28, t.s. 2. $\bullet$ S - Eoparastaffella macdermoti Devuyst \& Kalvoda, Ladyside Wood, Wo7, t.s. 39. $・$ T - Eoparastaffella ovalis Vdovenko, M2, Ladyside Wood, Wo7, t.s. 33. $\bullet$ U - Eoparastaffella vdovenkoae Devuyst \& Kalvoda, Ladyside Wood, Wo6, t.s. 17. $・ \mathrm{~V}-$ Eoparastaffella sp., Ladyside Wood, Wo7, t.s. 14. $・$ W - Eoparastaffella ex gr. interiecta Vdovenko, Ladyside Wood, Wo6, t.s. 38. - X - Eoparastaffella ex gr. florigena (Pronina), Ladyside Wood, Wo7, t.s. 9. • Y - Eoparastaffella ex gr. florigena (Pronina), juvenile, Ladyside Wood, Wo7, t.s. 48. Enlargement: 75×, V and Y 100x.

Figure 17. Typical foraminifers from Ladyside Wood in the North Staffordshire Basin. • A - Eoparastaffella ex gr. interiecta Vdovenko, Ladyside Wood, Wo7, t.s. 16. B - Eoparastaffella interiecta Vdovenko, Ladyside Wood, Wo11, t.s. 3. $\bullet$ C Eoparastaffella interiecta Vdovenko, Ladyside Wood 17, t.s. 9. $・$ D - Eoparastaffella ex gr. florigena (Pronina), Ladyside Wood, Wo3, t.s. 8. $・$ E - Eoparastaffella ex gr. florigena (Pronina), Ladyside Wood, Wo10, t.s. 15. $\bullet$ F - Eoparastaffella cf. restricta Postoyalko, Ladyside Wood, Wo10, t.s. 4. $\bullet$ G - Eoparastaffella ex gr. florigena (Pronina), Ladyside Wood, Wo3, t.s. 36. $\bullet$ - Eoparastaffella ex gr. florigena (Pronina), Ladyside Wood, Wo3, t.s. 17. I - Eoparastaffella ex gr. florigena (Pronina), Ladyside Wood, Wo6, t.s. 1. $・ \mathrm{~J}-$ Eoparastaffella ex gr. florigena (Pronina), Ladyside Wood, Wo10, t.s. 15. $\bullet \mathrm{K}-$ Eoparastaffella ex gr. florigena (Pronina), Ladyside Wood, Wo6, t.s. 14. $\bullet$ L - Eoparastaffella ex gr. florigena (Pronina), Ladyside Wood, Wo3, t.s. 37. $\bullet$ M - Eoparastaffella ex gr. florigena (Pronina), Ladyside Wood, Wo3, t.s. 21. $\bullet \mathrm{N}-$ Eoparastaffella cf. florigena (Pronina), Ladyside Wood, Wo7, t.s. 41. $\bullet$ - Eoparastaffella ex gr. florigena (Pronina), Ladyside Wood, Wo11, t.s. 5. $\bullet$ P - Eoparastaffella ex gr. florigena (Pronina), Ladyside Wood, Wo10, t.s. 6. $\bullet$ Q - Eoparastaffella ex gr. florigena (Pronina), Ladyside Wood, Wo22, t.s. 6. $\bullet$ R - Eoparastaffella ex gr. florigena (Pronina), Ladyside Wood, Wo7, t.s. 48 . $\bullet$ S - Eoparastaffella ex gr. florigena (Pronina), Ladyside Wood, Wo6, t.s. 27. $\bullet$ T - Eoparastaffella sp., Ladyside Wood, Wo3, t.s. 44. • U - Eoparastaffella ex gr. florigena (Pronina), Ladyside Wood, Wo3, t.s. 21.・V - Eoparastaffella ex gr. florigena (Pronina), Ladyside Wood, Wo3, t.s. 22. • W - Eoparastaffella ex gr. florigena (Pronina), Ladyside Wood, Wo3, t.s. 18. Enlargement: 75x, B 100x.

Figure 18. Typical foraminifers from Ladyside Wood in the North Staffordshire Basin. • A - Eoparastaffella ex gr. florigena (Pronina), Ladyside Wood, Wo3, t.s. 18. B - Eoparastaffella ex gr. florigena (Pronina), Ladyside Wood, Wo3, t.s. 26. $\bullet$ C - Eoparastaffella ex gr. florigena (Pronina), Ladyside Wood, Wo7, t.s. 44. $・$ D - Eoparastaffella ex gr. florigena (Pronina), Ladyside Wood, Wo10, t.s. 20. $\bullet$ E - Eoparastaffella ex gr. florigena (Pronina), Ladyside Wood, Wo28, t.s. 1. • F - Eoparastaffella ex gr. florigena (Pronina), Ladyside Wood, Wo6, t.s. 3. • G - Eoparastaffella ex gr. florigena (Pronina), Ladyside Wood, Wo7, t.s. 27. $\bullet \mathrm{H}-$ Eoparastaffella ex gr. florigena (Pronina), Ladyside Wood, Wo19, t.s. 5. $\bullet$ I - Eoparastaffella ex gr. florigena (Pronina), Ladyside Wood, Wo10, t.s. 4. $\bullet$ J Eoparastaffella ex gr.florigena (Pronina), Ladyside Wood, Wo28, t.s. 4. $\bullet$ - Eoparastaffella ex gr. florigena (Pronina), Ladyside Wood, Wo28, t.s. 1. - L - Eoparastaffella ex gr. florigena (Pronina), Ladyside Wood, Wo19, t.s. 3. - M - Eoparastaffella ex gr. florigena (Pronina), Ladyside Wood, Wo19, t.s. 1. $\bullet \mathrm{N}-$ Eoparastaffella cf. tummida (Pronina), Ladyside Wood, Wo7, t.s. 9. - O - Eoparastaffella tummida (Pronina), Ladyside Wood, Wo6, t.s. 16. $\bullet$ P - Eoparastaffella cf. tummida (Pronina), Ladyside Wood, Wo28, t.s. 5. - Q - Eoparastaffella ex gr. florigena (Pronina), Ladyside Wood, Wo3, t.s. 15. - R - Eoparastaffella sp., Ladyside Wood, Wo22, t.s. 1. - S - Eoparastaffella tummida (Pronina), Ladyside Wood, Wo3, t.s. 16. $・$ T - Eoparastaffella cf. tummida (Pronina), Ladyside Wood, Wo6, t.s. 26. $\cdot \mathrm{U}$ - Eoparastaffella cf. tummida (Pronina), Ladyside Wood, Wo28, t.s. 4. $\bullet \mathrm{V}-$ Eoparastaffella cf. tummida (Pronina), Ladyside Wood, Wo3, t.s. 45. -W - Eoparastaffella ex gr. florigena (Pronina), Ladyside Wood, Wo10, t.s. 6. Enlargement: 75×, E 50x. 

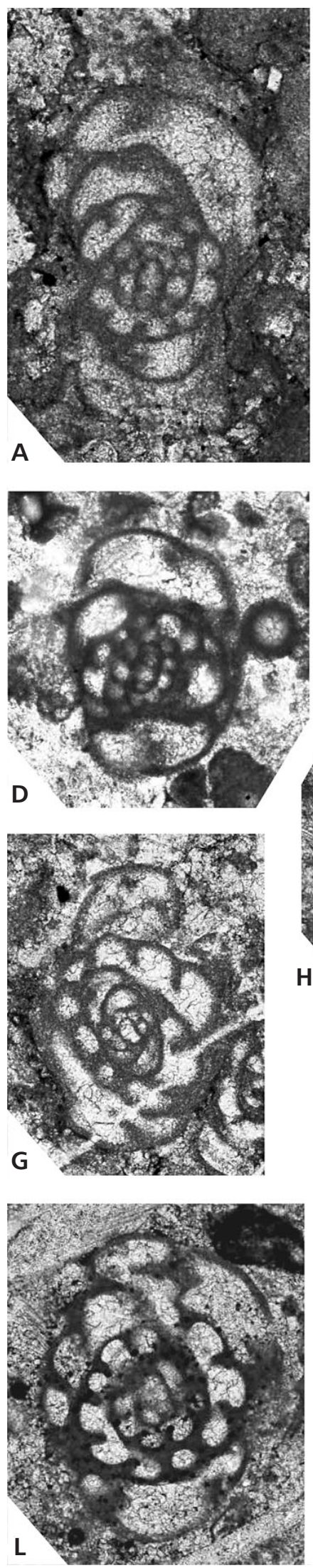
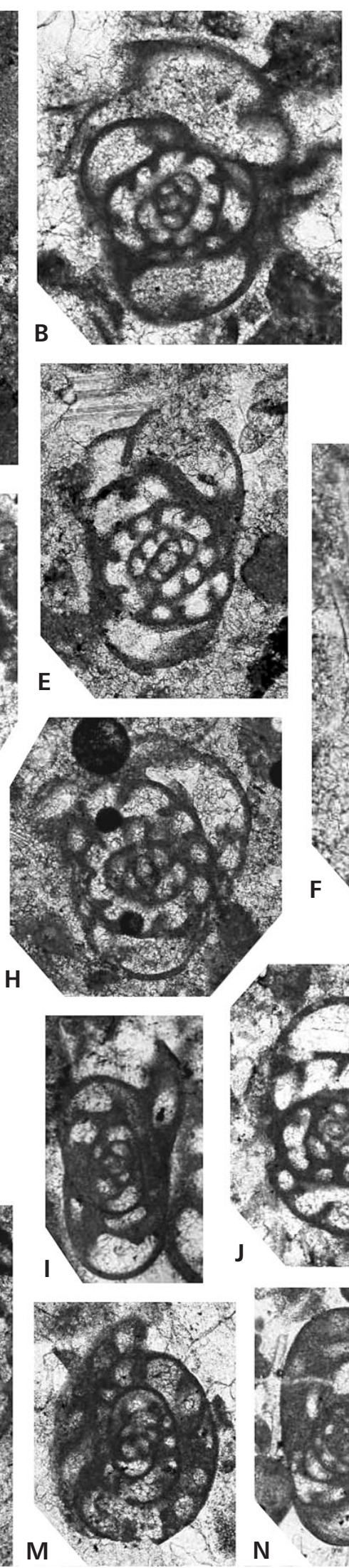
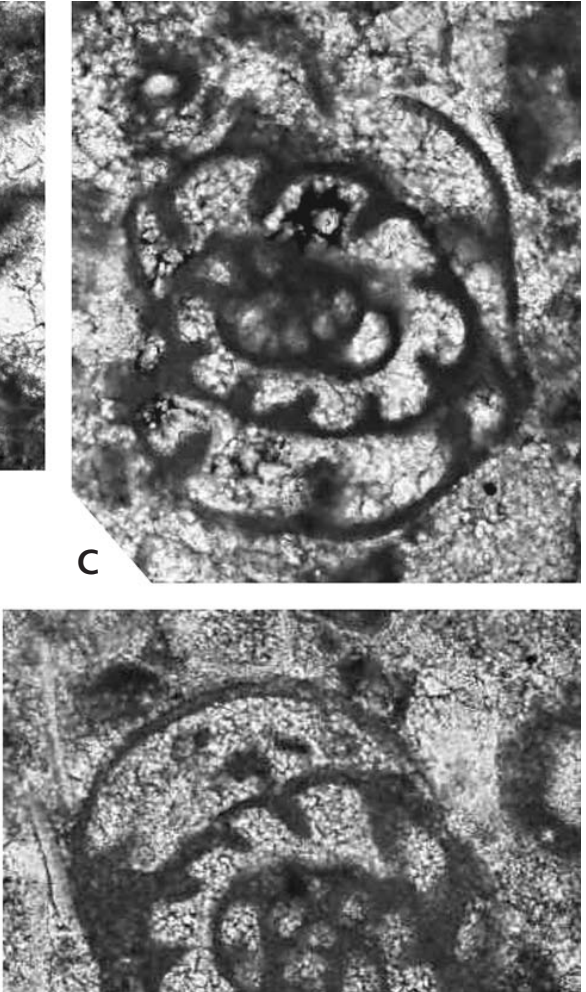

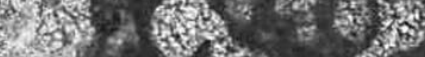
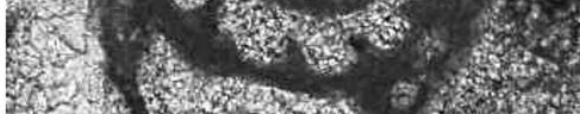

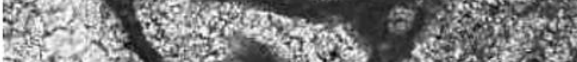
2. 1 .

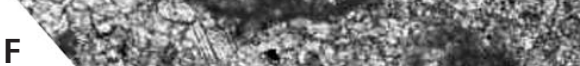
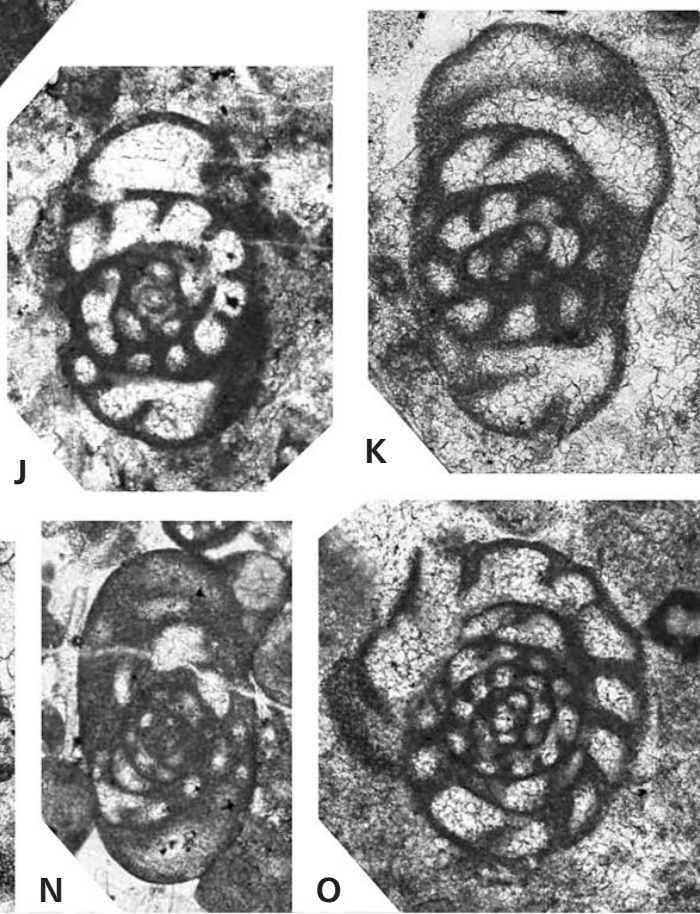

Figure 15. Typical foraminifers from Ladyside Wood in the North Staffordshire Basin. For explanation see page 516. 

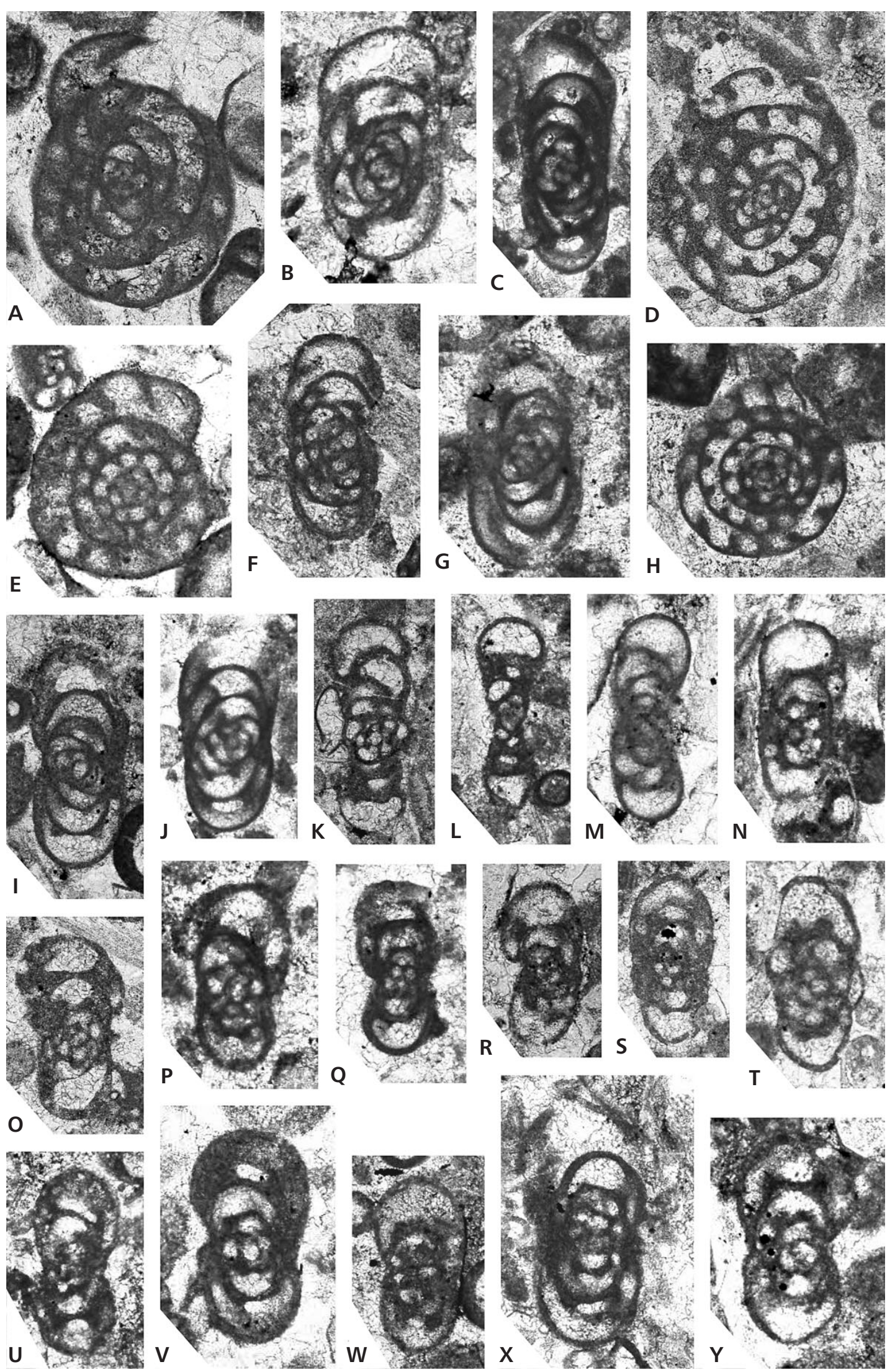

Figure 16. Typical foraminifers from Ladyside Wood in the North Staffordshire Basin. For explanation see page 516. 

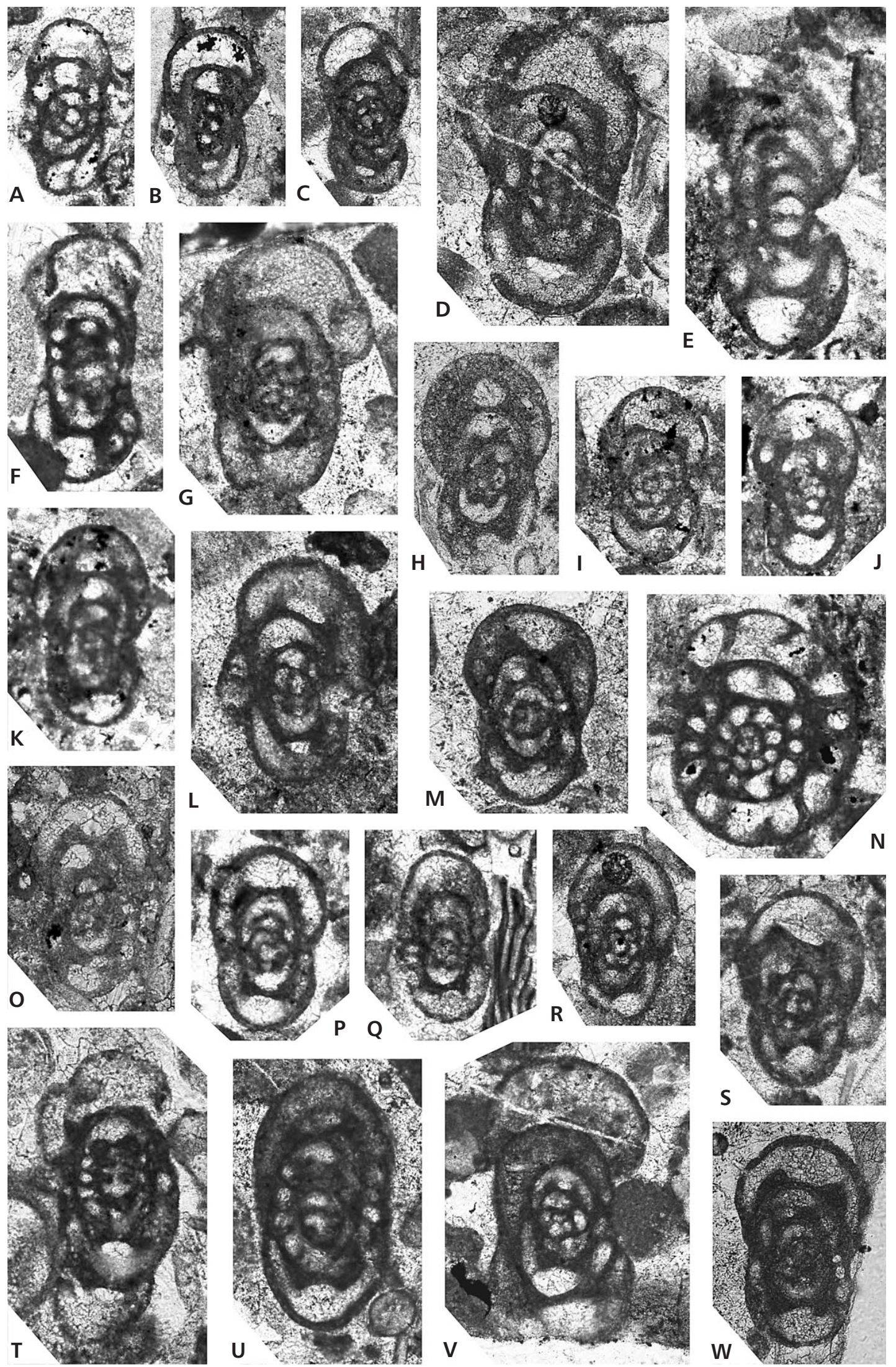

Figure 17. Typical foraminifers from Ladyside Wood in the North Staffordshire Basin. For explanation see page 516. 

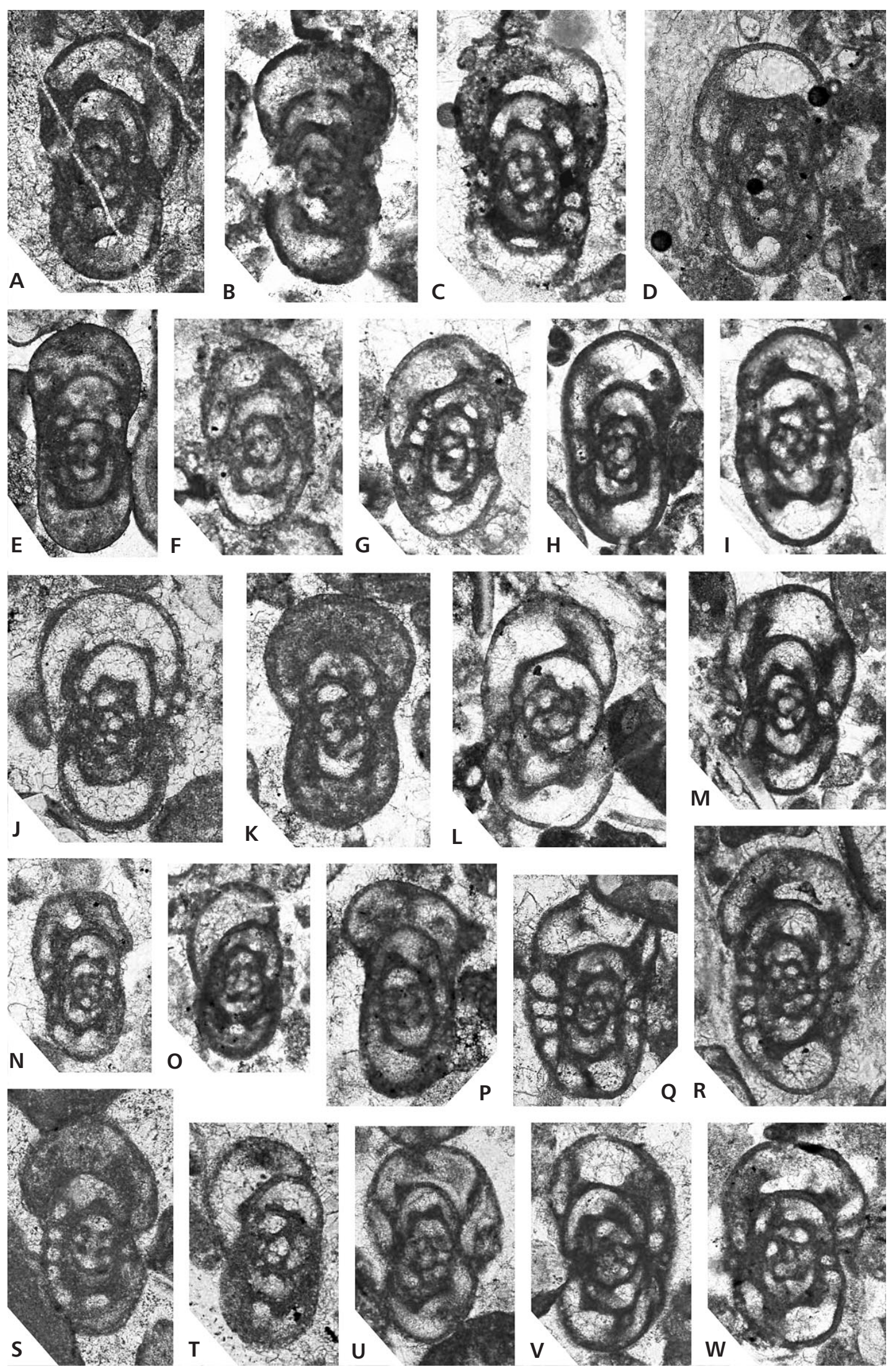

Figure 18. Typical foraminifers from Ladyside Wood in the North Staffordshire Basin. For explanation see page 516. 
The lack of diagnostic sections and the presence only of rare juveniles make it difficult to determine the level of Eoparastaffella entry. However, the presence of ?Eoparastaffella at the very base of the interval, and the fact that the first richer Eoparastaffella occurrence above is represented by upper MFZ8 taxa, indicate that most probably the interval corresponds to MFZ8. This interpretation is corroborated by the presence of Eosinopsis the FAD of which has not been traced below MFZ8 (Hance et al. 2011). These data clearly illustrate the problems in recognising the base of the Chadian which correlates with the base of MFZ8. The Waulsortian and peri-Waulsortian facies and spiculitic limestone are all quite unfavourable for Eoparastaffella (Lees 1997).

The first bioclastic layer B27 contains already L. gadukensis which follows both the occurrences of $E$. cf. interiecta and E. cf. vdovenkoae indicating close proximity of the T-V boundary. Higher up in B11 the entry of Eosinopsis solida so far reported only in Viséan MFZ9 is accompanied by a form transitional between Eoparastaffella ovalis M1 and M2. Similar forms enter just below the T-V boundary in the stratotype in Pengchong (Devuyst 2006). Above B11 richer foraminiferal associations occur especially in the growing number of bioclastic limestones at this level, and, in a parallel trend, foraminifers become more common in wackestones; this accords with the growing number of dasyclads present, which have been derived from more shallow water environments.

In sample B53 forms similar to the paratype of Paralysella schubertelloides (Bozorgnia 1973, pl. 13, fig. 10) occur, a form previously only recorded from the Viséan (Devuyst 2006, Kalvoda et al. 2011). The FAD of E. cf. simplex (B42) is preceded by the entries of Mediocris ex gr. mediocris, Eosinopsis solida and Paralysella schubertelloides whose ranges are still not so well established but which have been reported only in the Viséan.

The massive bed with sample P13 in the centre of the section contains only larger Eoparastaffella mostly of the variable E. florigena group together with a rich association of specimens Eosinopsis solida and Eosinopsis? ex gr. donica (Brazhnikova \& Rostovtseva) and the Paralysella schubertelloides. Above this bed, Eoparastaffella is represented mostly by the E. florigena and E. interiecta group, while E. simplex and E. ovalis M2 continue to be rare. Another important entry at this this level is the appearance of Paralysella ex gr. mediocriformis (Bozorgnia).

Above B4 there is an interval of micritic limestone in which foraminifers are both rare and stratigraphically unimportant. Bioclastic limestones at the top of the section contain advanced forms of Eoparastaffella with walls tran- sitional to Eostaffella (Conil et al. 1980; Groessens et al. 1982, 1964; Hance 1997; Devuyst 2006) including Eoparastaffella nalivkini (Malakhova). No archaediscids have been encountered at the base of the Hopedale Limestone in the sample B1. However, the presence of Paralysella cf. mutabiliformis Popova reported in the UstGrekhov horizon (MFZ10-11) of the Urals (Simonova 1975, Ivanova 2008, Kulagina 2011) may indicate the proximity of the Arundian.

\section{Ossom's Hill}

Figures 21-24

At Ossom's Hill the section starts in wackestone facies with only rare endothyrids represented mostly by small juveniles. As in other sections Endothyra and Spinobrunsiina are present. The first richer association comes from a well graded calciturbidite OH5 (Fig. 6). The presence of variable Eoparastaffella ex gr. florigena (the dominant form among Eoparastaffella), Eoparastaffella ovalis transitional between M1 and M2, Lysella gadukensis and Paralysella indicates close proximity to the T-V boundary. In the following samples OH6 juveniles dominate with Brunsia and Spinobrunsiina still very abundant. A Viséan MFZ9 age is indicated by the presence of E. asymmetrica and E. ovalis M2. In OH7, in addition to the Viséan indicators E. ovalis M2 and E. tumida, Tournayellida including Spinobrunsiina, Brunsia, Eblanaia michoti, Eblanaia sp. and Spinochernella brenklei are all present. S. brencklei is reported in Belgium only in MFZ4 (Devuyst \& Hance in Poty et al. 2006). Although E. michoti was formerly regarded as only late Tournaisian in Belgium, it has recently been reported from the early Viséan of the Urals by Kulagina (2011). The presence of E. michoti together with Viséan Eoparastaffella guides indicates a lower part of MFZ9. More abundant too is Bessiella and, a similar trend was encountered in $\mathrm{OH} 9$. The increased variability within the foraminiferal association at this level demonstrates derivation from different parts of the ramp as genera not usually found together are present (Eoparastaffella - Eblanaia). In $\mathrm{OH} 9$ the first representatives of the E. simplex group appear (E. simplex, E. iniqua Postoyalko) and the number of Spinobrunsiina and Brunsia decrease. There is a further sharp decline of these taxa in OH12 where Eoparastaffella species show the highest variability. Beside E. simplex the presence of E. evoluta, E. laciniosa and E. pseudochomata, together with Eoparastaffella in which the wall resembles Eostaffella, indicate a higher part of MFZ9. A similar foraminiferal association also characterises the last studied sample OH14.

Although different ontogenetic stages of $E$. ex gr. florigena dominate in the Ossom's Hill section, the Eoparastaffella association is more diverse than that at either the Ladyside Wood or Brown End Quarry sections. 


\section{South Wales - Mendip Shelf}

\section{Three Cliffs Bay}

Figures 19, 20

The upper part of the Friars Point Limestone is dolomitized and only few samples yielded foraminifers. The first sam- ple TB1 contained mostly small juveniles or fragments. Eoparastaffella is absent, an the MFZ8 age is indicated by other MFZ8 guide, namely Biseriella bristolensis and the late Tournaisian guides Darjella monilis and Elevenella parvula (Fig. 7).

In the lower part of the Gully Oolite below the palaeosol horizon Biseriella bristolensis is accompanied by advanced

Figure 19. Typical foraminifers from Ladyside Wood in the North Staffordshire Basin (A-W), and Three Cliffs Bay in the South Wales-Mendip Shelf (Y-AB). • A - Eoparastaffella ovalis Vdovenko, M1, Ladyside Wood, Wo6, t.s. 12. • B - Eoparastaffella sp., Ladyside Wood, Wo22, t.s. 6. - C - Eoparastaffella ovalis Vdovenko, M2, Ladyside Wood, Wo6, t.s. 25. • D - Eoparastaffella ovalis Vdovenko, M2, aberant form, Ladyside Wood, Wo6, t.s. 1. - E - Eoparastaffella cf. simplex Vdovenko, transitional form to Eoparastaffella ovalis Vdovenko, M2, Ladyside Wood, Wo6, t.s. 29. • F - Eoparastaffella ovalis Vdovenko, M1, Ladyside Wood 17, t.s. 11. • G - Eoparastaffella sp., Ladyside Wood, Wo12, t.s. 6. • H - Eoparastaffella ovalis Vdovenko, M1, Ladyside Wood, Wo6, t.s. 3. • I - Eoparastaffella sp., aberant form, Ladyside Wood, Wo22, t.s. 6. • J - Eoparastaffella sp., Ladyside Wood, Wo12, vt.s. 6. $• \mathrm{~K}$ - Eoparastaffella sp., Ladyside Wood, Wo19, t.s. 6. $\bullet$ L - Eoparastaffella sp., Ladyside Wood, Wo19, t.s. 1. • M - Eoparastaffella ex gr. simplex Vdovenko, Ladyside Wood, Wo22, t.s. 2. $・ \mathrm{~N}$ - Eoparastaffella ex gr. simplex Vdovenko, Ladyside Wood, Wo7, t.s. 12. $\bullet$ O - Eoparastaffella ex gr. simplex Vdovenko, Ladyside Wood, Wo10, t.s. 20. • P - Eoparastaffella cf. simplex Vdovenko, Ladyside Wood, Wo10, t.s. 22.・ Q - Eoparastaffella sp., Ladyside Wood, Wo12, t.s. 5. • R - Eoparastaffella asymmetrica Vdovenko, Ladyside Wood, Wo7, t.s. 1. • S - Eoparastaffella asymmetrica Vdovenko, Ladyside Wood, Wo7, t.s. 15. • T - Eoparastaffella asymmetrica Vdovenko, Ladyside Wood, Wo6, t.s. 27. • U - Eoparastaffella asymmetrica Vdovenko, Ladyside Wood, Wo7, t.s. 15. • V - Eoparastaffella asymmetrica Vdovenko, Ladyside Wood, Wo6, t.s. 40. • W - Eoparastaffella sp., Ladyside Wood, Wo22, t.s. 6. • X - Elevenella parvula (Bozorgnia), Three Cliffs Bay, TB1, t.s 1. • Y - Biseriella bristolensis (Reichel), Three Cliffs Bay, TB1, t.s. 4. - Z - Biseriella bristolensis (Reichel), Three Cliffs Bay, TB1, t.s. 2. • AA - Lysella gadukensis Bozorgnia, Three Cliffs Bay, TB20, t.s. 9. - AB - Eoparastaffella ex gr. florigena (Pronina), Three Cliffs Bay, TB6, t.s. 21. Enlargement: A-K, N, P-X, AA-AB 75×, L-M, O, Y-Z 100×.

Figure 20. Typical foraminifers from Three Cliffs Bay in the South Wales-Mendip Shelf. • A - Eoparastaffella ex gr. florigena (Pronina), Three Cliffs Bay, TB10, t.s. 5. • B - Eoparastaffella ex gr. florigena (Pronina), Three Cliffs Bay, TB10, t.s. 5. • C - Eoparastaffella ex gr. interiecta Vdovenko, Three Cliffs Bay, TB6, t.s. 30. - D - Eoparastaffella vdovenkoae Devuyst \& Kalvoda transitional form to Eoparastaffella macdermoti Devuyst \& Kalvoda, Three Cliffs Bay, TB6, t.s. 4. • E - Eoparastaffella ovalis Vdovenko, M1, Three Cliffs Bay, TB6, t.s. 1. • F - Eoparastaffella vdovenkoae Devuyst \& Kalvoda, Three Cliffs Bay, TB6, t.s. 24. • G - Eoparastaffella ovalis Vdovenko, M2, Three Cliffs Bay 10, t.s. 3. • H - Eoparastaffella macdermoti Devuyst \& Kalvoda, Three Cliffs Bay, TB10, t.s. 16. • I - Eoparastaffella ovalis Vdovenko, M1, Three Cliffs Bay, TB10, t.s. 5. • J - Eoparastaffella ovalis Vdovenko, M1, Three Cliffs Bay, TB6, t.s. 13. $\bullet \mathrm{K}-$ Eoparastaffella simplex Vdovenko, Three Cliffs Bay, TB20, t.s. 14. $\bullet$ L - Eoparastaffella asymmetrica Vdovenko, Three Cliffs Bay, TB20, t.s. 6. • M - Eoparastaffella ovalis Vdovenko, M1, Three Cliffs Bay, TB7, t.s. 6. $\bullet$ N - Eoparastaffella sp., Three Cliffs Bay, TB20, t.s. 1. • O - Eoparastaffella simplex Vdovenko, Three Cliffs Bay, TB21, t.s. 6. • P - Eoparastaffella ovalis Vdovenko, M1, Three Cliffs Bay, TB10, t.s. 13.・Q - Eoparastaffella cf. macdermoti Devuyst \& Kalvoda, Three Cliffs Bay, TB10, t.s. 5. • R - Eoparastaffella sp., Three Cliffs Bay, TB10, t.s. 6. $\bullet \mathrm{S}-$ Eoparastaffella ovalis Vdovenko, M2, Three Cliffs Bay, TB10, t.s. 7. $\bullet$ T - Eoparastaffella simplex Vdovenko, Three Cliffs Bay, TB21, t.s. 5. Enlargement: A, B-D, F, H, J-K, M-O, R-T 75×; B, E, G, I, L, P-Q 100×.

Figure 21. Typical foraminifers from Ossom's Hill in the North Staffordshire Basin. • A - Plectogyranopsis regularis (Rauser-Chernousova), Ossom's Hill, OH7, t.s. 20. • B - Plectogyranopsis regularis (Rauser-Chernousova), Ossom's Hill, OH7, t.s. 11. • C - Eblanaia michoti (Conil \& Lys), Ossom's Hill, OH7, t.s. 1. • D - Spinochernella brencklei Conil \& Lys, Ossom's Hill, OH7, t.s. 10. • E - Spinobrunsiina cf. implicata (Conil \& Lys), Ossom's Hill, OH7, t.s. 18. • F - Spinochernella spinosa (Chernysheva), Ossom's Hill, OH5, t.s. 1. • G - Spinobrunsiina lexhyi Conil \& Hance, Ossom's Hill, OH8, t.s. 3. • H - Spinobrunsiina lexhyi Conil \& Hance, Ossom's Hill, OH7, t.s. 9. • I - Ammarchaediscus sp., Ossom's Hill, OH25, t.s. 2. • J - Bessiella cf. leusoniae Conil, Ossom's Hill, OH6, t.s. 1. • K - Bessiella legrandi (Conil \& Hance), Ossom's Hill, OH8, t.s. 12. $\bullet$ L - Omphalotis ex gr. chariessa (Conil \& Lys), Ossom's Hill, OH7, t.s. 18. • M - Eosinopsis primaeva Vachard \& Hance, Ossom's Hill, OH7, t.s. 2. Enlargement: 75×, M 50×.

Figure 22. Typical foraminifers from Ossom's Hill in the North Staffordshire Basin. • A - Bessiella rectiformis (Bogush \& Yuferev), Ossom's Hill, OH5, t.s. 12. • B - Bessiella rectiformis (Bogush \& Yuferev), Ossom's Hill, OH9, t.s. 6. • C-Bessiella rectiformis (Bogush \& Yuferev), Ossom's Hill, OH9, t.s. 2. •D - Paralysella sp., Ossom's Hill, OH7, t.s. 8. E - Lysella gadukensis Bozorgnia, Ossom's Hill, OH7, t.s. 17. • F- Lysella gadukensis Bozorgnia, Ossom's Hill, OH7, t.s. $3 . \bullet \mathrm{G}-$ Paralysella sp., Ossom's Hill, OH 6, t.s. 5. $\bullet \mathrm{H}-$ Bessiella legrandi Conil \& Hance, Ossom's Hill, OH8, t.s. $12 . \bullet \mathrm{I}-$ Lysella gadukensis Bozorgnia, Ossom's Hill, OH7, t.s. 4. J- Paralysella sp., Ossom's Hill, OH9, t.s. 5. • K - Eoparastaffella ex gr. florigena (Pronina), Ossom's Hill, OH7, t.s. 19. • L - Eoparastaffella ex gr. florigena (Pronina), Ossom's Hill, OH5, t.s. 1. • M - Eoparastaffella ex gr. florigena (Pronina), Ossom's Hill, OH5, t.s. 6. - N - Eoparastaffella ex gr. florigena (Pronina), Ossom's Hill, OH6, t.s. 6. • O - Eoparastaffella ex gr. florigena (Pronina), Ossom's Hill, OH5, t.s. 5. - P - Eoparastaffella ex gr. florigena (Pronina), Ossom's Hill, OH5, t.s. 4. • Q - Eoparastaffella ex gr. florigena (Pronina), Ossom's Hill, OH6, t.s. 8. - R - Eoparastaffella sp., Ossom's Hill, OH7, t.s. 9. • S - Eoparastaffella ex gr. interiecta Vdovenko, Ossom's Hill, OH6, t.s. 7. 

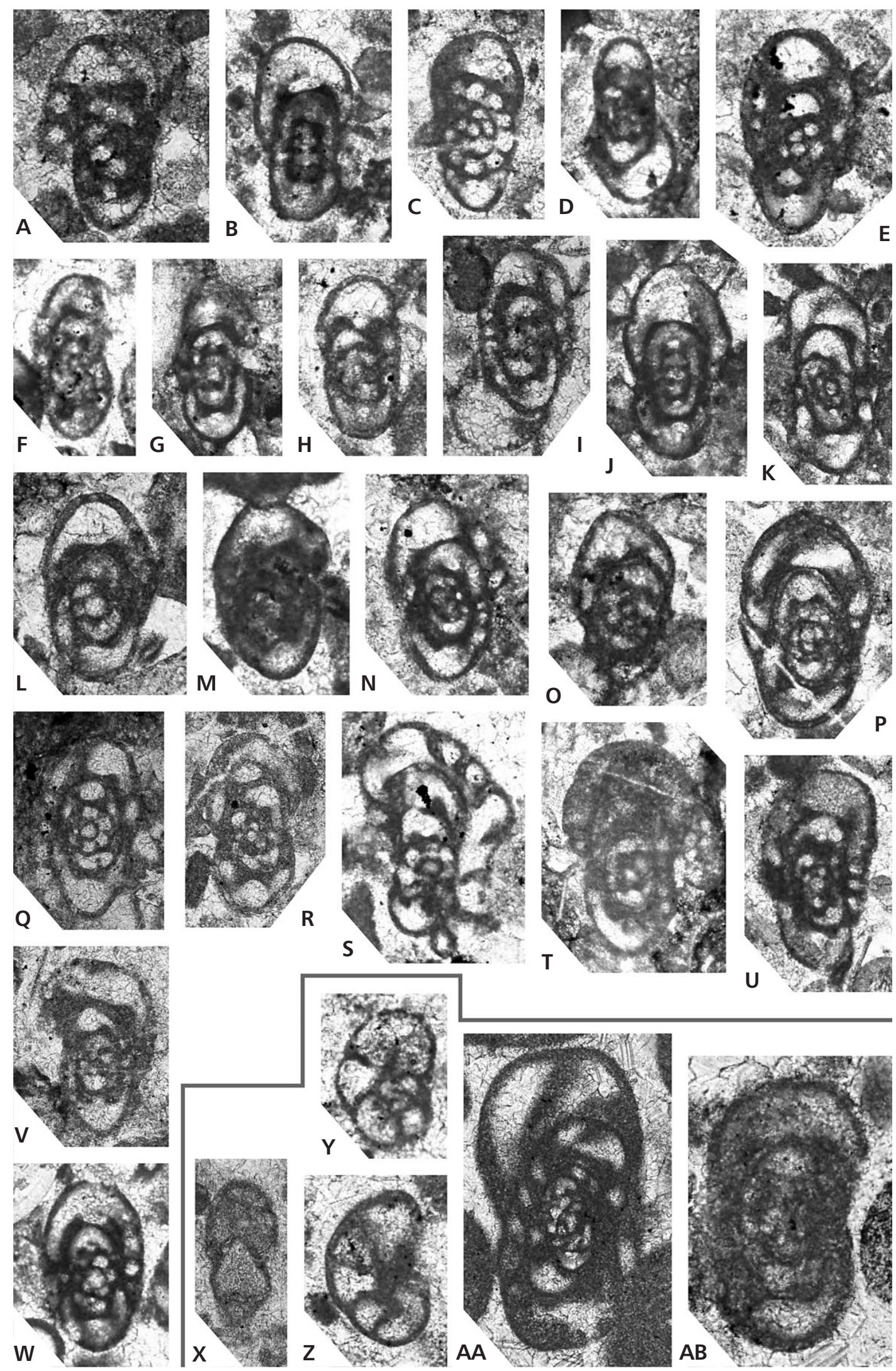

Figure 19. Typical foraminifers from Ladyside Wood in the North Staffordshire Basin (A-W), and Three Cliffs Bay in the South Wales-Mendip Shelf (Y-AB). For explanation see page 522. 

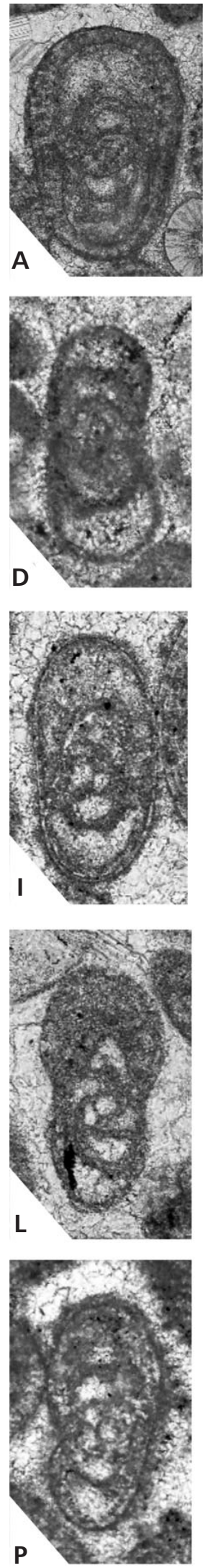
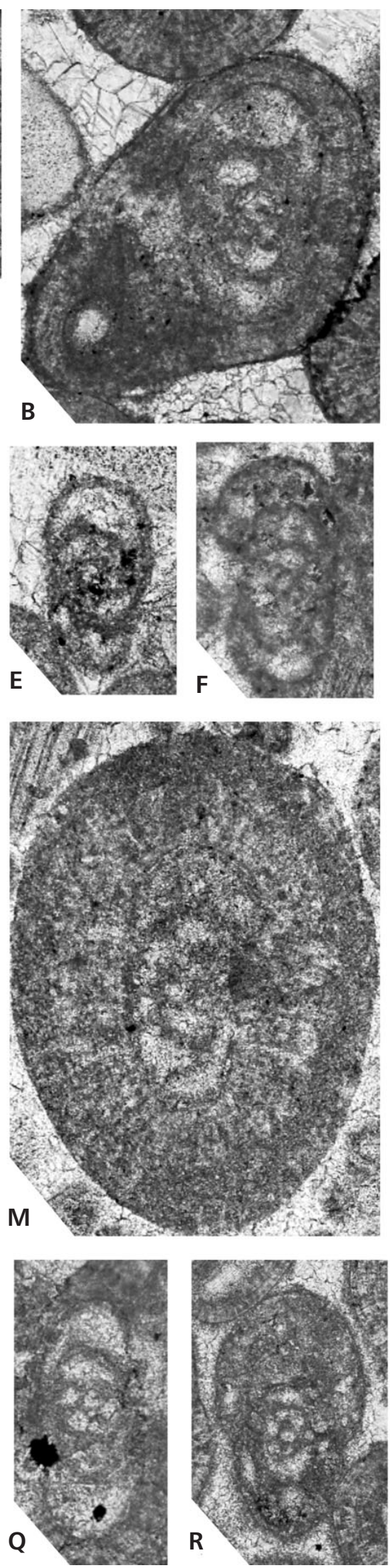
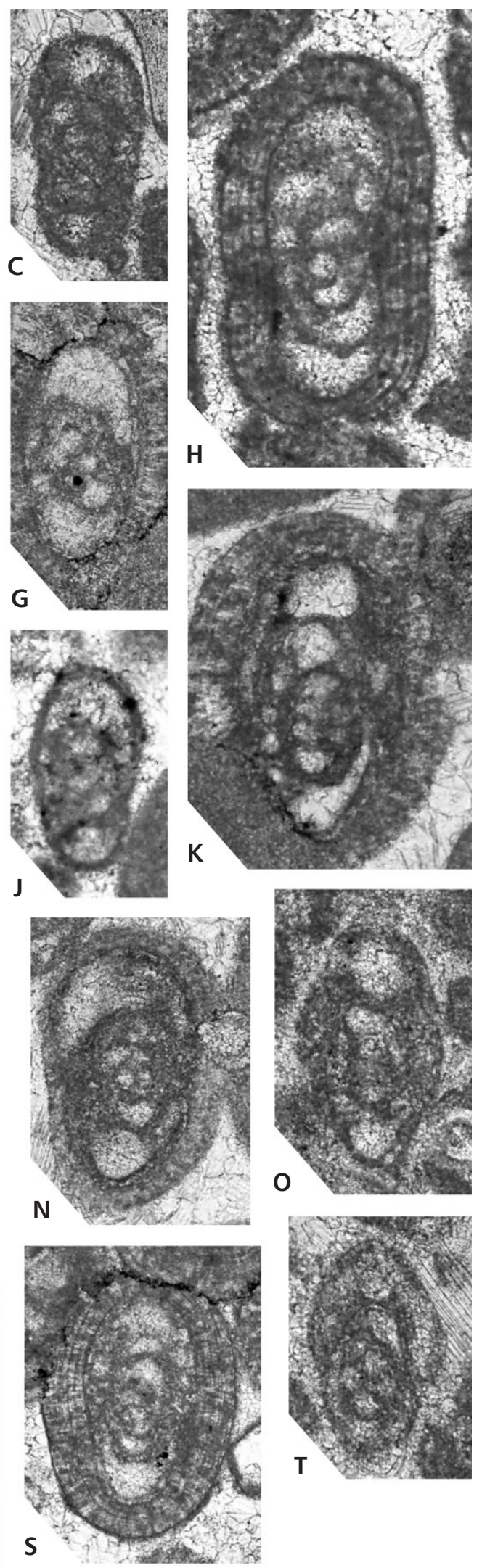

Figure 20. Typical foraminifers from Three Cliffs Bay in the South Wales-Mendip Shelf. For explanation see page 522. 

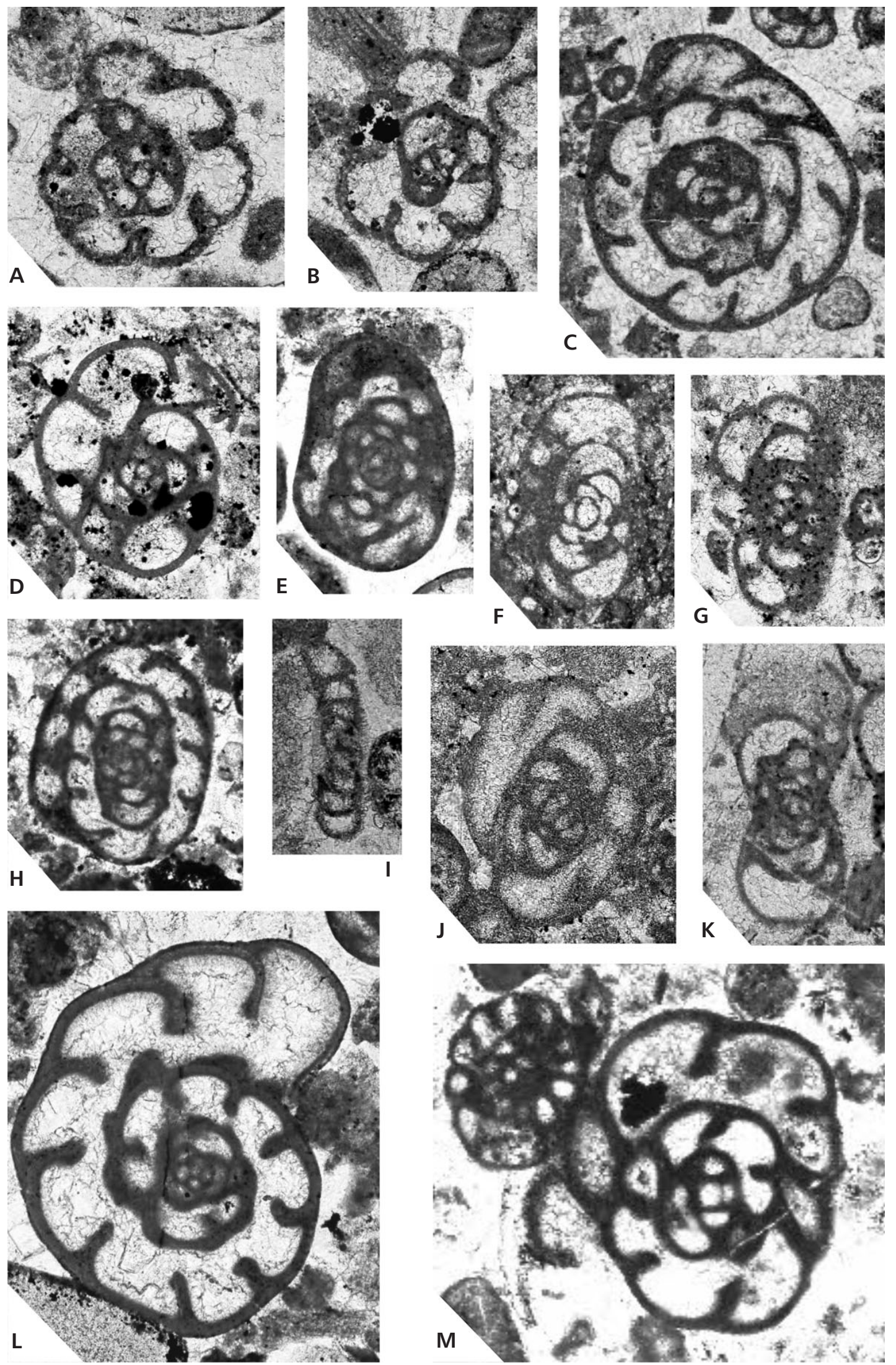

Figure 21. Typical foraminifers from Ossom's Hill in the North Staffordshire Basin. For explanation see page 522. 

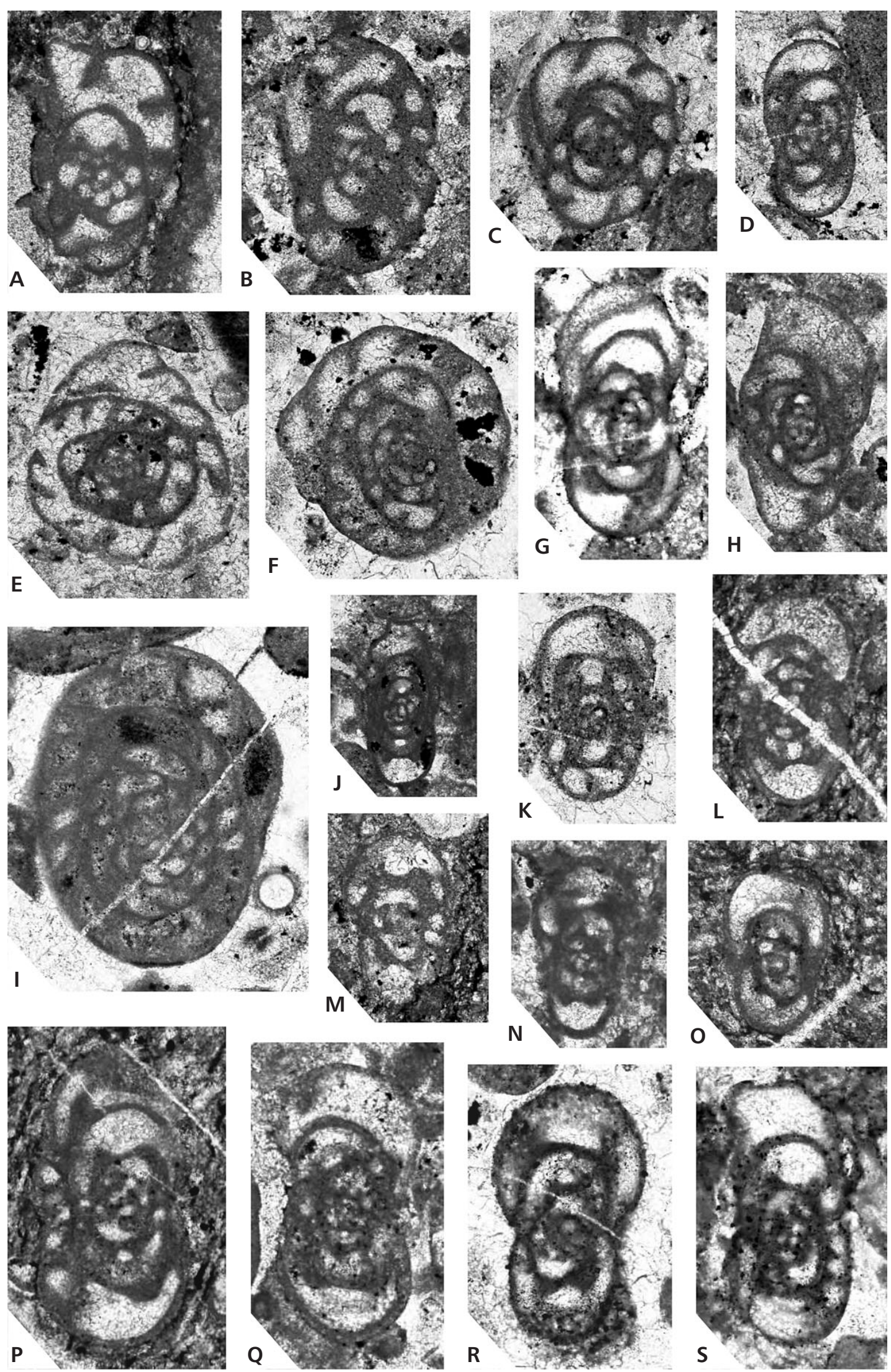

Figure 22. Typical foraminifers from Ossom's Hill in the North Staffordshire Basin. For explanation see page 522. 
Eoparastaffella, namely E. ex gr. florigena, E. macdermoti Devuyst \& Kalvoda, E. vdovenkoae Devuyst \& Kalvoda and E. ovalis M3 which indicate the uppermost part of MFZ8. In contrast to the North Staffordshire Basin localities, members of the E. ovalis group dominate at Three Cliffs Bay. In the upper part of the Gully Oolite a Viséan MFZ9 age is indicated by the presence of E. ovalis M2, E. asymmetrica M1, E. tummida (sample TB10). As in sections in the North Staffordshire Basin, Spinobrunsiina is abundant but E. simplex is quite rare. Eoparastaffella is however the dominant foraminifer here and forms the cores of most ooids.

The Caswell Bay Mudstone contains only poorly preserved foraminifers such that the distinction between MFZ9 and MFZ10 is not possible. The base of the High Tor Limestone is characterised by a widespread occurrence of E. simplex and the advanced forms Eoparastaffella pseudochomata and E. asymmetrica M2 (Devuyst 2006). The very rare presence of archaediscids represented by Uralodiscus and Glomodiscus indicates a higher part of MFZ10 or even a lower MFZ11.

\section{Ogmore (Vale of Glamorgan)}

As the Arundian age of the Caswell Bay Mudstone was only supposed (Waters et al. 2009) and it was not possible to determine it in Three Cliffs Bay, the locality was chosen in addition. The Viséan age of the upper part of the Gully Oolite is indicated by presence of MFZ9 guide E. simplex and E. asymmetrica (Fig. 8). The sample Go3 about $7 \mathrm{~m}$ above the base of the Caswell Bay Mudstone contained relatively rich foraminiferal association with important Arundian guides Viseidiscus sp., Glomodiscus sp. and Glomodiscus cf. biarmicus Malakhova indicating already higher part of MFZ10 (Fig. 8).

\section{Discussion}

Brown End Quarry and Ladyside Wood both provide important British sections across the $\mathrm{T}-\mathrm{V}$ boundary, but the better section is arguably that at Brown End Quarry because it displays the evolutionary lineages of Eoparastaffella from the latest Tournaisian to the early Viséan and it has good conodont record (Chisholm et al. 1988). The section therefore appears to be much better for the definition of the base of the Chadian than the stratotype in Chatburn Bypass (George et al. 1976).

By way of contrast however, the Ladyside Wood section is a better section for understanding problems of the Chadian biostratigraphy. Here the absence of Eoparastaffella in the latest Tournaisian is followed by the entry of advanced basal Viséan Eoparastaffella species such as E. tummida, E. asymmetrica and E. ovalis M2. In order to locate the T-V boundary accurately in these sec- tions it is important to consider the microfacies. In the North Staffordshire Basin and the Dublin Basin (Kalvoda et al. 2011) Eoparastaffella is absent or very rare in the heterozoan dominated associations. However, in the absence of Eoparastaffella other taxa can prove useful to approximate the boundary. The FAD of Mediocris which is coincident with the base of MFZ8 may provide an alternative guide (Devuyst \& Hance in Poty et al. 2006) even though in the sections studied here it occurs at a much higher stratigraphic level (Figs 4, 5). Another possibility is the entry of Plectogyranopsis regularis reported only from the latest Tournaisian and Viséan in the Urals (Postoyalko 1975, Simonova 1975) and from the Viséan of the Pengchong GSSP (Hance et al. 2011). Interestingly, in the Craven Basin, both Plectogyranopsis regularis and Mediocris have been reported in the Chadian stratotype at Chatburn (Riley 1995). Given the broadly similar deep ramp settings of the Craven and North Staffordshire basins, this raises the possibility that even though Eoparastaffella is absent at Chatburn, as at Ladyside Wood, limestones of MFZ8, that is of Chadian age, may be present at Chatburn. In this context it is important to note that the entry of richer foraminiferal associations above the Waulsortian and peri-Waulsortian facies in the British Isles is strongly facies controlled and that only the late Chadian has a distinctive biostratigraphic signature (Riley 1990, 1993, 1995). In order to resolve such stratigraphic difficulties the best solution would be to equate the base of the Chadian with the base of the Viséan (Somerville 2008). However, given that E. simplex is quite rare in the Chadian of the British Isles other important Viséan guides such as E. ovalis M2, E. asymmetrica and E. tummida are required in defining the position of this important boundary.

Our study of Eoparastaffella evolution in the early Viséan MFZ9 of England and Wales has shown that higher parts of the zone are characterized by the entry of more advanced species such as Eoparastaffella restricta, E. laciniosa, E. pseudochomata and E. evoluta. This is in accord with the data from Ireland (Kalvoda et al. 2011), the Urals and the Donetz Basin (Postoyalko 1975, Stepanova et al. 2008, Brazhnikova \& Vdovenko 1973). In addition, forms with a wall transitional between Eoparastaffella and Eostaffella occur, as they do in Belgium and at the Pengchong stratotype (Conil \& Naum 1977, Devuyst 2006, Hance 1997, Hance et al. 2011). Candidates for the distinction of this higher part of MFZ9 are Eoparastaffella nalivkini (Malakhova) (Ivanova 2008, Malakhova 1975, Simonova 1975) and Plectogyranopsis convexus. Also promising are species of Paralysella despite some taxonomic difficulties (Hance et al. 2011), but the stratigraphic potential and precise level of the FADs of these species have still to be tested.

In the North Staffordshire Basin the boundary between the Milldale Limestone and the overlying Hopedale 
Limestone or Ecton Limestone lies within the upper part of MFZ9, thus within the Chadian. However, the entry of the first archaediscids is not a very reliable criterion for the detection of the Arundian. The discontinuous and very rare presence of the first archaediscids documented in the Dublin Basin (Kalvoda et al. 2011) indicates that their occurrence is facies controlled (Gallagher 1998). Such difficulties are, however, not exceptional as similar problems are encountered in Belgium (Hance et al. 1981, Hance 1988) and at the Pengchong stratotype (Devuyst 2006). Consequently, the definition of the base of the Arundian by the FADs of Planoarchaediscus or Ammarchaediscus should be supplemented by other non archaediscid taxa to minimize the effects of facies control (Simpson \& Kalvoda 1987) and calibrated by geophysical (gamma ray spectrometry) and geochemical means (isotopes of $\mathrm{O}, \mathrm{C}$ and $\mathrm{Sr}$ ).
In South Wales the T-V boundary is closely followed by a distinctive mid-Avonian unconformity and thicknesses of limestones corresponding to MFZ9 and 10 are rather reduced. The shoreface Gully Oolite, however, yielded quite numerous Eoparastaffella associations showing the lineage from Eoparastaffella ovalis to Eoparastaffella simplex crucial for the T-V boundary definition (Fig. 7). For the first time the Arundian age (MFZ10) of the overlying Caswell Bay Mudstone can be confirmed by foraminifers.

The biostratigraphic analyses of the Tournaisian to early Viséan carbonate ramp and basin successions of the South Wales-Mendip Shelf (SWMS) and the North Staffordshire Basin (NSB) were also correlated independently using gamma ray spectrometry (GRS) and magnetic susceptibility (MS). The sedimentary facies and petrophysical log patterns show a robust ramp-to-basin correlation and a

Figure 23. Typical foraminifers from Ossom's Hill in the North Staffordshire Basin. • A - Eoparastaffella ex gr. florigena (Pronina), Ossom's Hill, OH7, t.s. 22. • B - Eoparastaffella ex gr. florigena (Pronina), Ossom's Hill, OH12, t.s. 2. • C - Eoparastaffella sp., Ossom's Hill, OH14, t.s. 3. - D - Eoparastaffella ex gr. florigena (Pronina), Ossom's Hill, OH12, t.s. 3. E - Eoparastaffella ex gr. florigena (Pronina), Ossom's Hill, OH7, t.s. 19. $\bullet \mathrm{F}$ - Eoparastaffella ex gr. florigena (Pronina), Ossom's Hill, OH14, t.s. 7. • G - Eoparastaffella ex gr. florigena (Pronina), Ossom's Hill, OH14, t.s. 7. - H - Eoparastaffella aff. laciniosa Postoyalko, Ossom's Hill, OH12, t.s. 1. • I - Eoparastaffella ex gr. florigena (Pronina), Ossom's Hill, OH14, t.s. 4. - J - Eoparastaffella sp., Ossom's Hill, OH12, t.s. 2. $\bullet \mathrm{K}$ - Eoparastaffella sp., Ossom's Hill, OH14, t.s. 1. • L - Eoparastaffella macdermoti Devuyst \& Kalvoda, Ossom's Hill, OH7, t.s. 25. $\bullet$ M - Eoparastaffella sp., Ossom's Hill, OH14, t.s. 6. $・ \mathrm{~N}-$ Eoparastaffella restricta Postoyalko, Ossom's Hill, OH14, t.s. 5. • O - Eoparastaffella sp., Ossom's Hill, OH9, t.s. 1. • P - Eoparastaffella ovalis Vdovenko, M3, Ossom's Hill, OH7, t.s. 5. $\cdot \mathrm{Q}$ - Eoparastaffella sp., Ossom's Hill, OH12, t.s. 3. $\bullet \mathrm{R}$ - Eoparastaffella ovalis Vdovenko, M2, Ossom's Hill, OH5, t.s. 12. $\mathrm{S}-$ Eoparastaffella ovalis Vdovenko, M2, Ossom's Hill, OH6, t.s. 4. • T - Eoparastaffella sp., Ossom's Hill, OH14, t.s. 4. • U - Eoparastaffella ovalis Vdovenko, M2, Ossom's Hill, OH6, t.s. 5. • V - Eoparastaffella ovalis Vdovenko, M2, Ossom's Hill, OH9, t.s. 6. $\bullet$ W - Eoparastaffella pseudochomata Vdovenko, Ossom's Hill, $\mathrm{OH} 12$, t.s. 3. $・ \mathrm{X}$ - Eoparastaffella asymmetrica Vdovenko, Ossom's Hill, OH6, t.s. 3. Enlargement: 75x.

Figure 24. Typical foraminifers from Ossom's Hill (A-P) and Brown End (Q-W) in the North Staffordshire Basin. • A - Eoparastaffella asymmetrica Vdovenko, Ossom's Hill, OH9, t.s. 4. • B - Eoparastaffella asymmetrica Vdovenko, Ossom's Hill, OH14, t.s. 1. • C - Eoparastaffella sp., Ossom's Hill, OH12, t.s. 7. • D - Eoparastaffella pseudochomata Vdovenko, Ossom's Hill, OH12, t.s. 1. • E - Eoparastaffella sp., Ossom's Hill, OH12, t.s. 5. - F - Eoparastaffella asymmetrica Vdovenko, Ossom's Hill, OH9, t.s. 1. • G - Eoparastaffella iniqua Postoyalko, Ossom's Hill, OH9, t.s. 1. $\bullet \mathrm{H}$ - Eoparastaffella evoluta Vdovenko, Ossom's Hill, OH12, t.s. 2. • I - Eoparastaffella sp., Ossom's Hill, OH9, t.s. 6. • J - Eoparastaffella cf. nalivkini (Malakhova), Ossom's Hill, OH12, t.s. 2. • K - Eoparastaffella ex gr. florigena (Pronina), Ossom's Hill, OH12, t.s. 1. • L - Eoparastaffella sp., Ossom's Hill, OH14, t.s. 3. • M - Eoparastaffella simplex Vdovenko, Ossom's Hill, OH9, t.s. 4. • N - Eoparastaffella sp., Ossom's Hill, OH14, t.s. 2. - O - Eoparastaffella ex gr. simplex Vdovenko Ossom's Hill, OH9, t.s. 1. • P - Eoparastaffella cf. nalivkini (Malakhova), Ossom's Hill, OH14, t.s. 4. - Q - Plectogyranopsis regularis (Rauser-Chernousova), Brown End, B1, t.s. 4. • R - Plectogyranopsis regularis (Rauser-Chernousova), Brown End, B16, t.s. 3. - S - Plectogyranopsis regularis (Rauser-Chernousova), Brown End, B45, t.s. 6. • T - Plectogyranopsis regularis (Rauser-Chernousova), Brown End, B4, t.s. 2. • U - Plectogyranopsis regularis (Rauser-Chernousova), Brown End, B42, t.s. 6. • V - Plectogyranopsis regularis (Rauser-Chernousova), Brown End, B58, t.s. 2. •W - Endospiroplectammina venusta (Vdovenko), Brown End, B42, t.s. 6. Enlargement: 75×, T 100×.

Figure 25. Typical foraminifers from Brown End in the North Staffordshire Basin. • A - Spinochernella brencklei Conil \& Lys, Brown End, B44, t.s. 4. - B - Spinochernella brencklei Conil \& Lys, Brown End, B58, t.s. 3. • C - Conilites dinantii (Conil \& Lys), Brown End, B43, t.s. 4. • D - Florennella amplissima Conil, Brown End, P13, t.s. 8. • E - Elevenella parvula (Bozorgnia), Brown End, P7, t.s. 1. • F - Paradainella sp., Brown End, P13, t.s. 3. - G - Spinobrunsiina lexhyi Conil \& Hance, Brown End, B42, t.s. 3. • H - Bessiella sp., Brown End, B58, t.s. 3. • I - Spinobrunsiina vachardi Conil, Brown End, B4, t.s. 5. • J - Spinobrunsiina lexhyi Conil \& Hance, Brown End, B27, t.s. 2. • K - Bessiella legrandi Conil \& Hance, Brown End, P13, t.s. 9. - L - Florennella stricta (Conil \& Lys), Brown End, P13, t.s. 1. • M - Bessiella legrandi Conil \& Hance, Brown End, P13, t.s. 1. • N - Bessiella limburgensis Conil \& Vieselet, Brown End, B11, t.s. 5. • O - Lysella gadukensis Bozorgnia, Brown End, B4, t.s. 1. • P - Lysella gadukensis Bozorgnia, Brown End, B45, t.s. 1. $\bullet$ Q - Paradainella dainelliformis Brazhnikova \& Vdovenko, Brown End, B43, t.s. 3. • R - Bessiella limburgensis Conil \& Vieselet, Brown End, B4, t.s. 3. Enlargement: 75×, C 50×. 

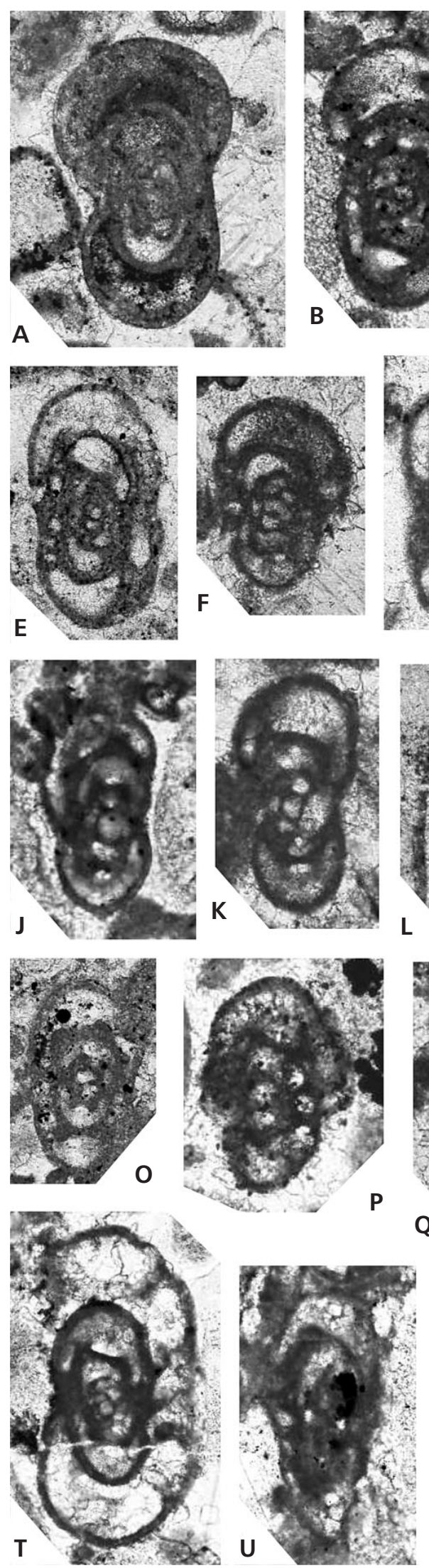
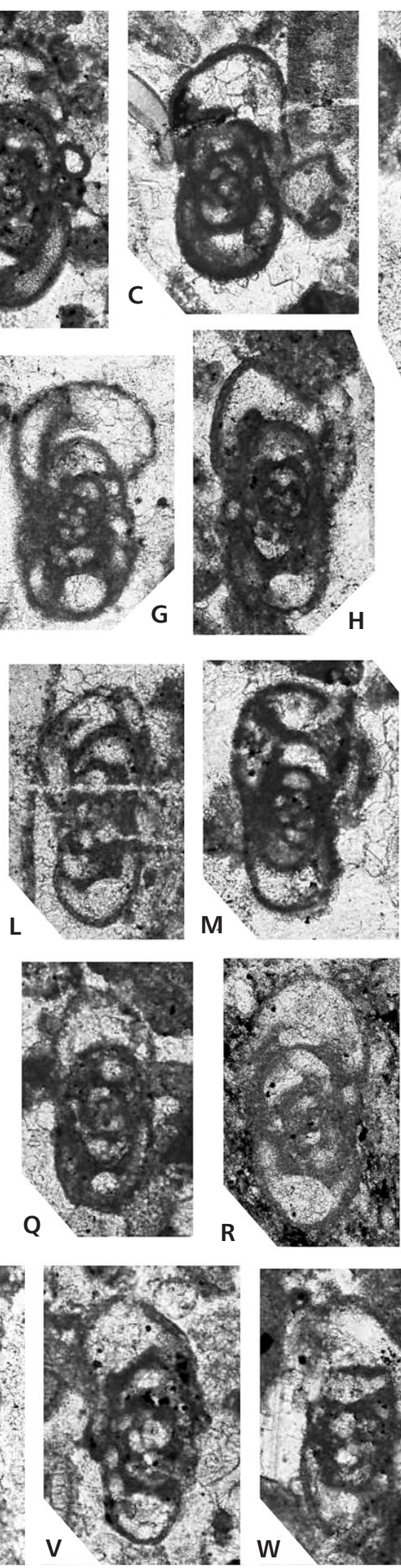
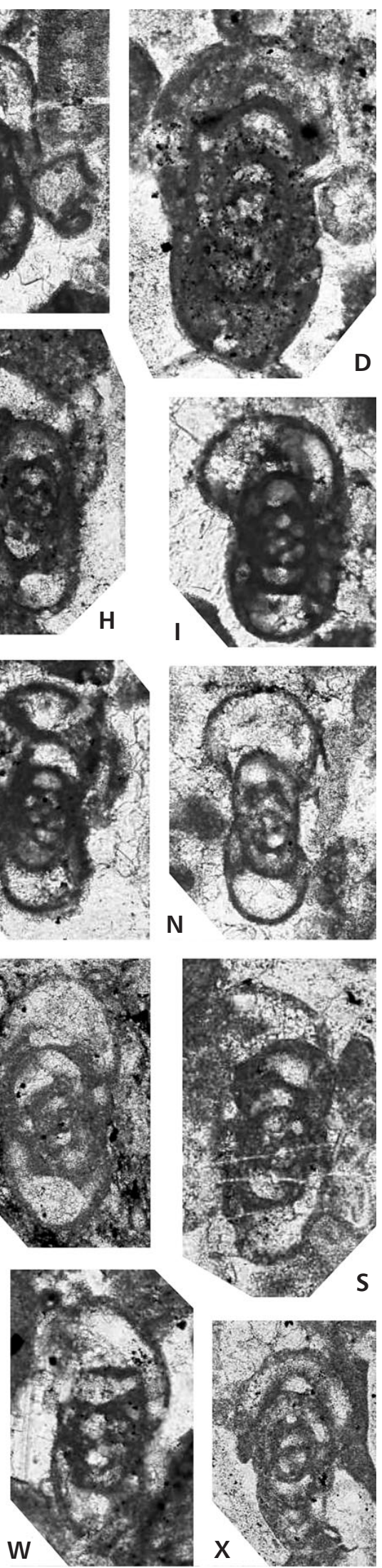

Figure 23. Typical foraminifers from Ossom's Hill in the North Staffordshire Basin.For explanation see page 528. 

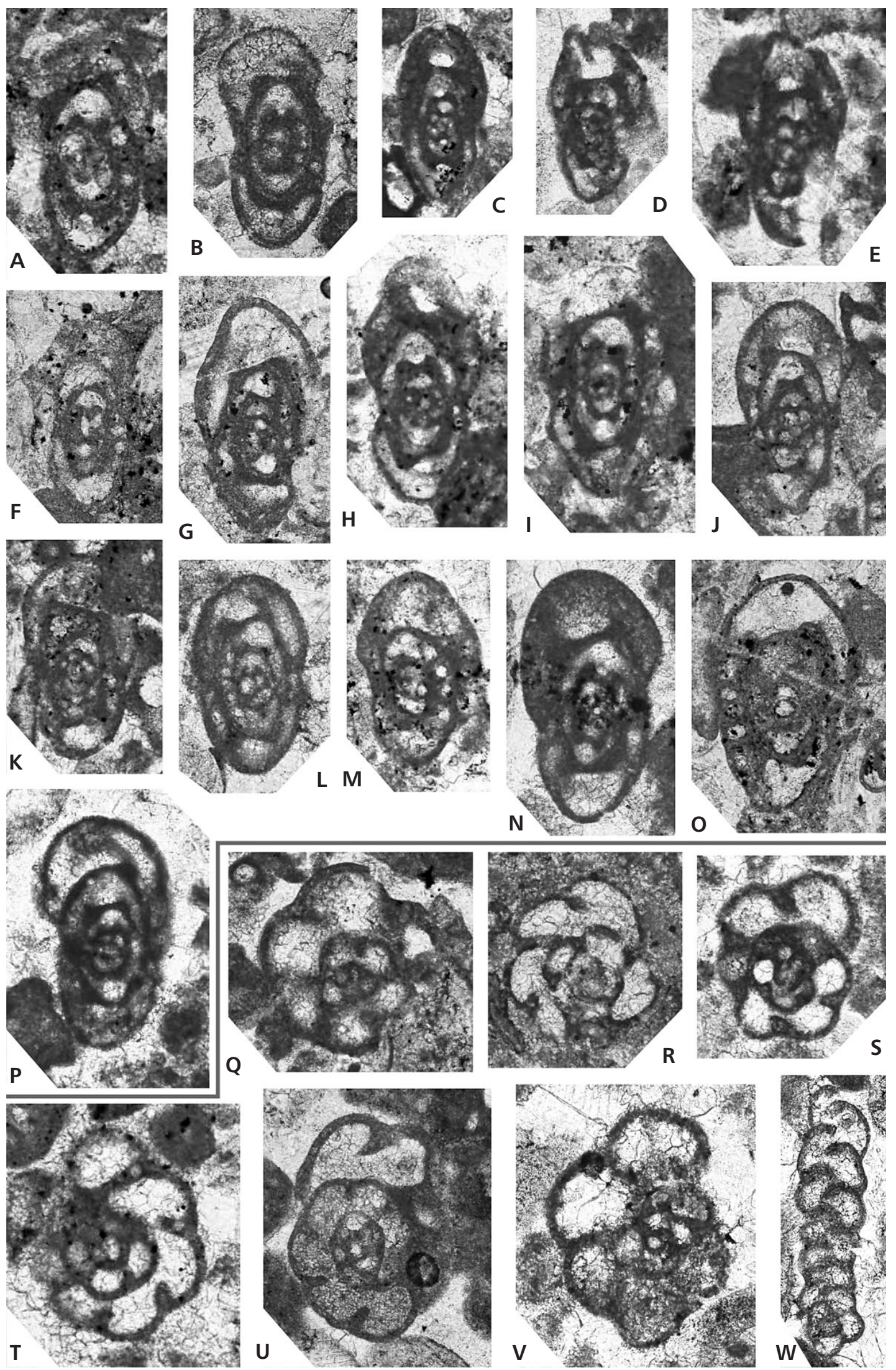

Figure 24. Typical foraminifers from Ossom's Hill (A-P) and Brown End (Q-W) in the North Staffordshire Basin. For explanation see page 528. 

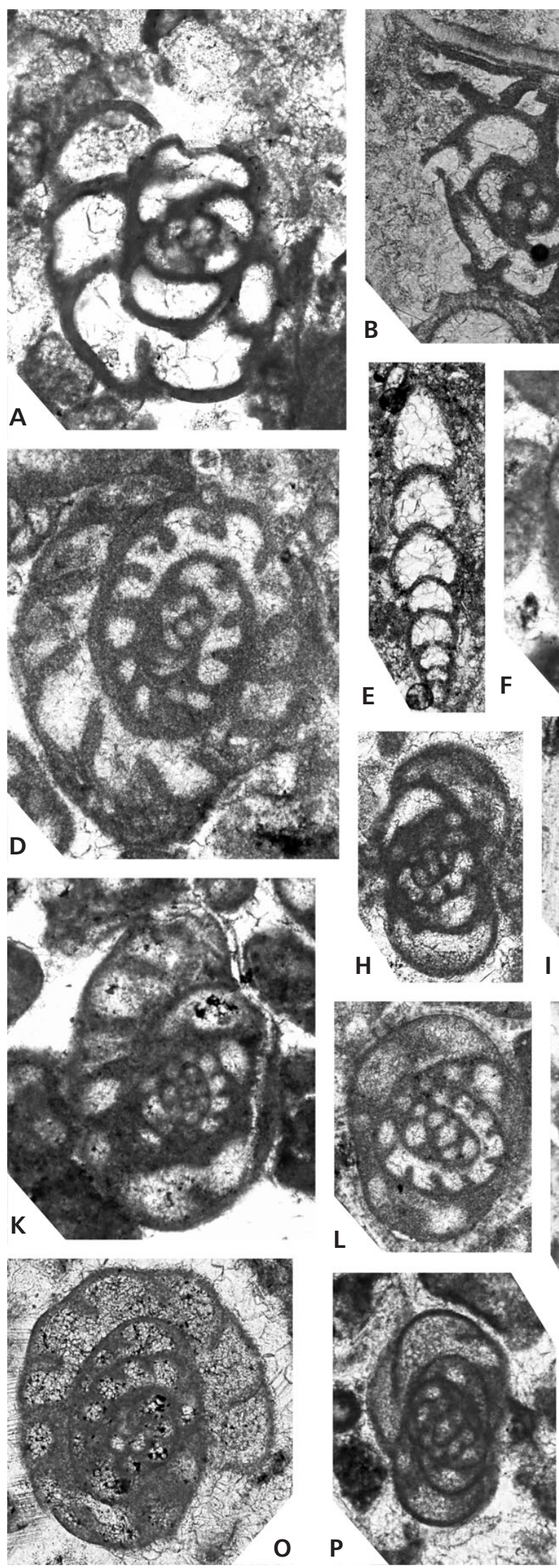
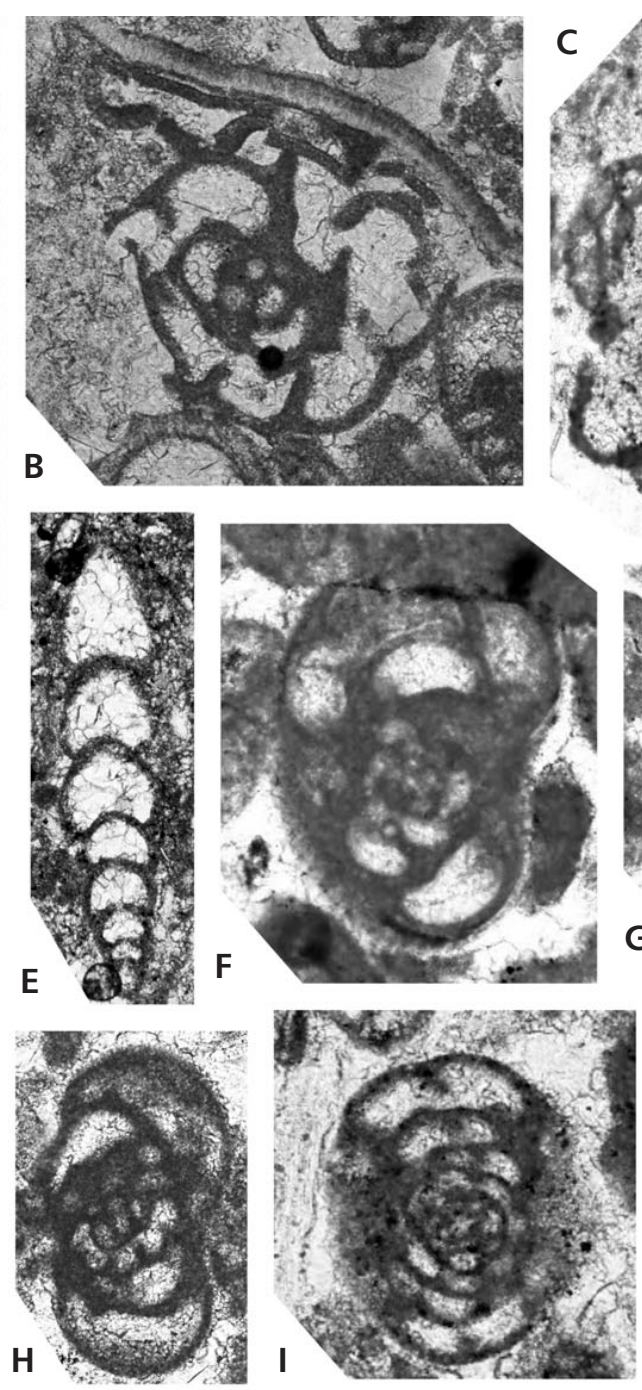

$G$ wasd 8 in
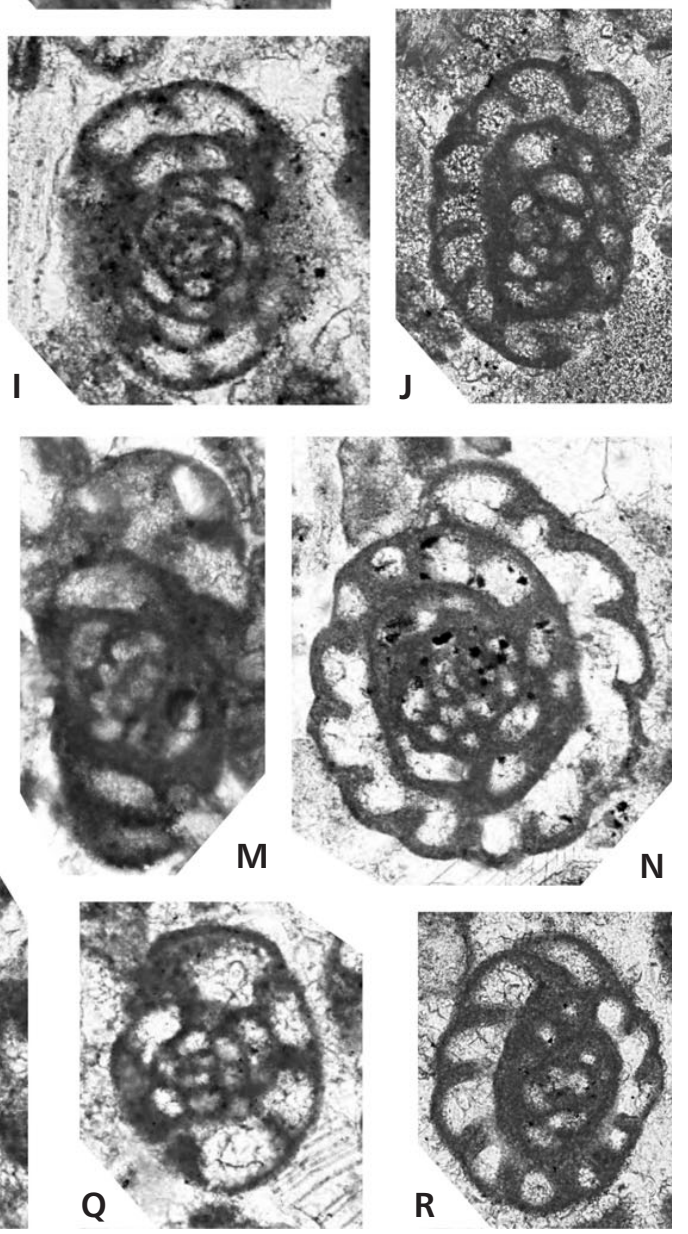

Figure 25. Typical foraminifers from Brown End in the North Staffordshire Basin. For explanation see page 528. 
distinct, late Tournaisian to early Viséan regressive-totransgressive cycle with a prominent sequence boundary located close to the T-V stage boundary (Hance et al. 2002) in both the NSB and SWMS sections (Fig. 9). A turnover to decreasing CGR (clay gamma ray) values calculated from the concentrations of K and Th (see Rider 1999) in the upper part of MFZ 9 in the NSB, which roughly coincides with the base of the Ecton Limestone and the Hopedale Limestone, represents another sequence boundary capping the lower Viséan depositional sequence. This correlates with the gap associated with the mid-Avonian sequence boundary between the Chadian Gully Oolite and Arundian Caswell Bay Mudstone in the Three Cliffs Bay and Ogmore section (SWMS).

The sea-level changes close to the T-V boundary are possibly of glacio-eustatic origin (Kalvoda 1986, 1989, 2002; Ross \& Ross 1987). The conodont apatite $\delta^{18} \mathrm{O}$ values suggest that a potential glaciation persisted from the Tournaisian to the Viséan with a distinct cooling at the T-V boundary (Buggish et al. 2008) which is in accord with a major positive $\delta^{13} \mathrm{C}$ shift recognized in the T-V boundary stratotype area in South China (Qie et al. 2011).

The foraminiferal associations from the Milldale Limestone in the North Staffordshire Basin occur within proximal and distal carbonate turbidites. A lower degree of transportation is expected in the Friars Point Limestone in Three Cliffs Bay (Ramsay 1987, Wright 1986) but the upper dolomitized part yielded only few foraminifers. Foraminifers are however relatively abundant in the inner ramp Gully Oolite where they form the cores of most ooids.

Even though most of the foraminiferal associations in the NSB are a product of different environments their ecology represents an important issue. Heterozoan dominated facies in the latest Tournaisian limestones may indicate either the supply from the middle part of the ramp or from shallow water parts of the inner ramp influenced by higher nutrient levels (Carannante et al. 1988, Schlager 2003,

Figure 26. Typical foraminifers from Brown End in the North Staffordshire Basin. - A - Lysella gadukensis Bozorgnia, Brown End, B53, t.s. 4. - B - Lysella gadukensis Bozorgnia, Brown End, B43, t.s. 3. • C - Lysella gadukensis Bozorgnia, Brown End, B7, t.s. 17. • D - Lysella gadukensis Bozorgnia, Brown End, B53, t.s. 5. • E - Lysella gadukensis Bozorgnia, Brown End, B7, t.s. 3. • F - Lysella gadukensis Bozorgnia, Brown End, B43, t.s. 1. • G - Lysella gadukensis Bozorgnia, Brown End, B12, t.s. 2. • H - Paralysella sp., Brown End, P13, t.s. 5. • I - Lysella gadukensis Bozorgnia, Brown End, B53, t.s. 4. - J - Lysella gadukensis Bozorgnia, Brown End, B4, t.s. 2. - K - Darjella monilis Malakhova, Brown End, B36, t.s. 1. - L - Eoparastaffella interiecta Vdovenko, Brown End, B53, t.s. 5. • M - Eoparastaffella macdermoti Devuyst \& Kalvoda, Brown End, B7, t.s. 1. - N - Eoparastaffella macdermoti Devuyst \& Kalvoda, Brown End, B11, t.s. 1. • O - Eoparastaffella macdermoti Devuyst \& Kalvoda transitional to Eoparastaffella vdovenkoae Devuyst \& Kalvoda, Brown End, B4, t.s. 1. • P - Eoparastaffella vdovenkoae Devuyst \& Kalvoda, Brown End, B53, t.s. 5. - Q - Eoparastaffella ex gr. interiecta Vdovenko, Brown End, B5, t.s. 6. • R - Eoparastaffella macdermoti Devuyst \& Kalvoda, Brown End, B5, t.s. 2. - S - Eoparastaffella macdermoti Devuyst \& Kalvoda, Brown End, B5, t.s. 1. • T - Eoparastaffella ex gr. florigena (Pronina), Brown End, B45, t.s. 5. - U - Eoparastaffella sp., Brown End, P13, t.s. 15. Enlargement: 75×.

Figure 27. Typical foraminifers from Brown End in the Staffordshire Basin. - A - Lysella gadukensis Bozorgnia, Brown End, B7, t.s. 10. - B - Paralysella sp., Brown End, B53, t.s. 4. $・$ C - Eoparastaffella ex gr. florigena (Pronina), Brown End, B1, t.s. 3. • D - Eosinopsis solida Vachard \& Hance, Brown End, P13, t.s. 2. • E - Paralysella sp., Brown End, B27, t.s. 2. • F-Paralysella, Brown End, P13, t.s. 8. • G- Eosinopsis primaeva Vachard \& Hance, Brown End, B36, t.s. 1. $\bullet \mathrm{H}$ - Eosinopsis primaeva Vachard \& Hance, Brown End, P13, t.s. 6. $\bullet$ I - Eoparastaffella ex gr. florigena (Pronina), Brown End, B7, t.s. 19. • J - Eoparastaffella sp., Brown End, B5, t.s. 6. $\bullet \mathrm{K}-$ Lysella gadukensis Bozorgnia, Brown End, P13, t.s. 4 . $\bullet$ L - Eoparastaffella ex gr. florigena (Pronina), Brown End, B7, t.s. 19. • M - Eoparastaffella sp., Brown End, B54, t.s. 6. N - Lysella gadukensis Bozorgnia, Brown End, B7, t.s. 10. $\bullet \mathrm{O}$ - Eoparastaffella ovalis Vdovenko, M1, Brown End, B27, t.s. 1. • P - Eoparastaffella vdovenkoae Devuyst \& Kalvoda, Brown End, B27, t.s. 1. - Q - Eoparastaffella vdovenkoae Devuyst \& Kalvoda, Brown End, B 20, t.s. 4. • R - Eoparastaffella macdermoti Devuyst \& Kalvoda, Brown End, B45, t.s. 5. - S - Eoparastaffella sp., Brown End, B7, t.s. 19. Enlargement: 75×, M and P 100×.

Figure 28. Typical foraminifers from Brown End in the North Staffordshire Basin. • A - Eoparastaffella ex gr. interiecta Vdovenko, Brown End, B43, t.s. 4. • B - Eoparastaffella vdovenkoae Devuyst \& Kalvoda, Brown End, B54, t.s. 6. • C - Eoparastaffella vdovenkoae Devuyst \& Kalvoda transitional to Eoparastaffella macdermoti Devuyst \& Kalvoda, Brown End, B27, t.s. 1. • D - Eoparastaffella ovalis Vdovenko, M2, Brown End, B58, t.s. 2. - E - Eoparastaffella ovalis Vdovenko, M2, Brown End, B6, t.s. 10. - F - Eoparastaffella cf. simplex Vdovenko, Brown End, B44, t.s. 1. - G-Eoparastaffella pseudochomata Vdovenko, Brown End, B1, t.s. 4. • H - Eoparastaffella sp., Brown End, B58, t.s. 4. • I - Eoparastaffella sp., Brown End, B5, t.s. 2. $\bullet$ J - Eoparastaffella cf. simplex Vdovenko, Brown End, B58, t.s. 2. • K - Eoparastaffella sp., Brown End, B4, t.s. 4 . $\bullet$ L - Eoparastaffella sp., Brown End, B1, t.s. 1. • M - Eoparastaffella sp., Brown End, B1, t.s. 3. • N - Eoparastaffella sp., Brown End, B1, t.s. 4. • O - Eoparastaffella sp., Brown End, B44, t.s. 4. • P - Eoparastaffella sp., Brown End, B58, t.s. 3.・Q-Eoparastaffella sp., Brown End, B1, t.s. 4. • R - Eoparastaffella sp., Brown End, B1, t.s. 4. $\bullet$ S - Eoparastaffella sp., Brown End, B1, t.s. 4. • T - Eoparastaffella sp., Brown End, B6, t.s. 17. • U - Eoparastaffella sp., Brown End, B58, t.s. 2. • V - Eoparastaffella sp., Brown End, B44, t.s. 1. • W - Eoparastaffella sp., Brown End, B58, t.s. 4. $・$ X - Eoparastaffella sp., Brown End, B7, t.s. 4. • Y - Eoparastaffella sp., Brown End, B12, t.s. 3. • Z - Eoparastaffella sp., Brown End, B58, t.s. 2. • AA - Eoparastaffella ex gr. florigena (Pronina), Brown End, B45, t.s. 6. - AB - Eoparastaffella sp., Brown End, B1, t.s. 5. Enlargement: 75×, E, G, S, V 100×. 

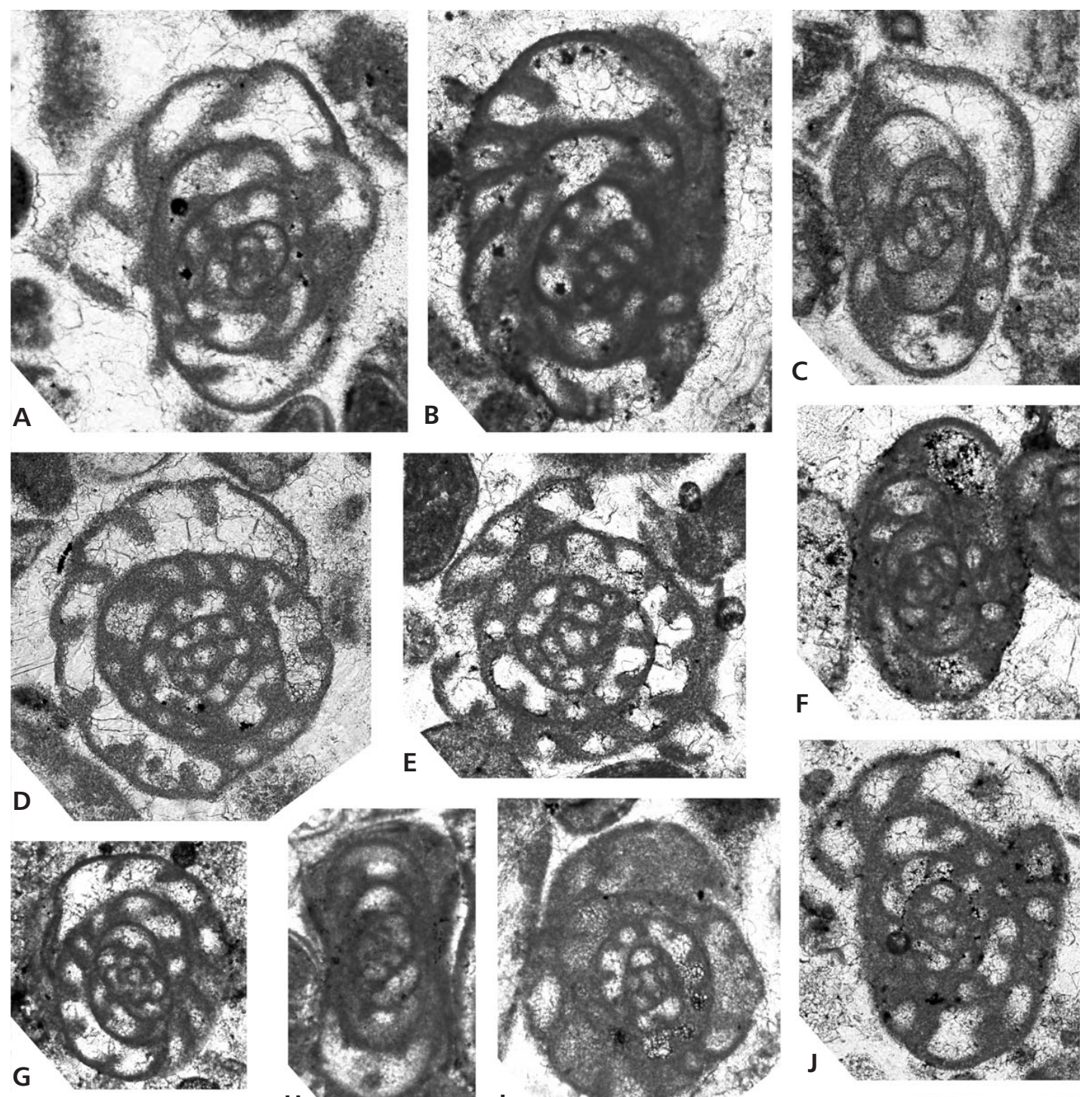

$\mathbf{F}$
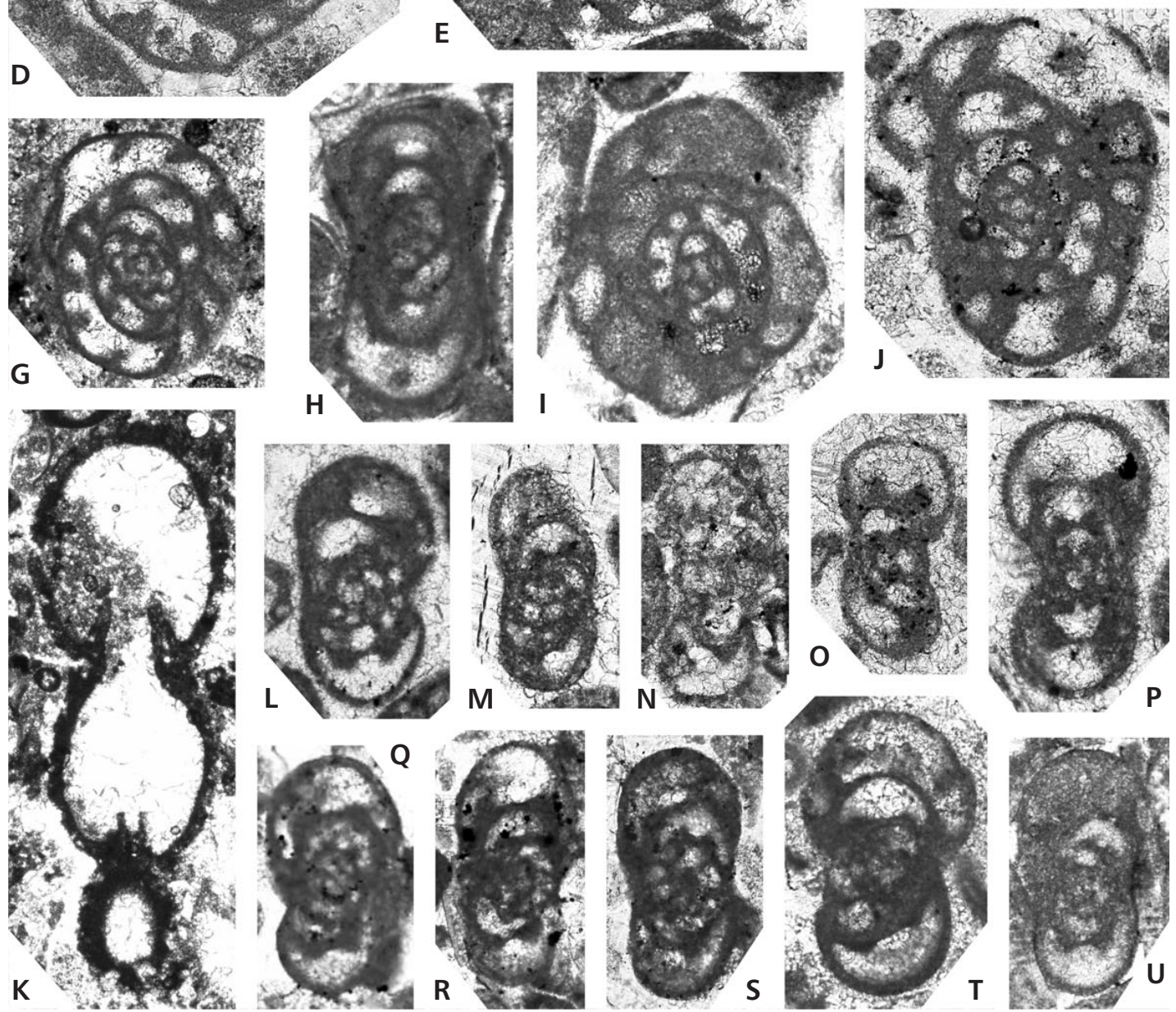

Figure 26. Typical foraminifers from Brown End in the North Staffordshire Basin. For explanation see page 532. 

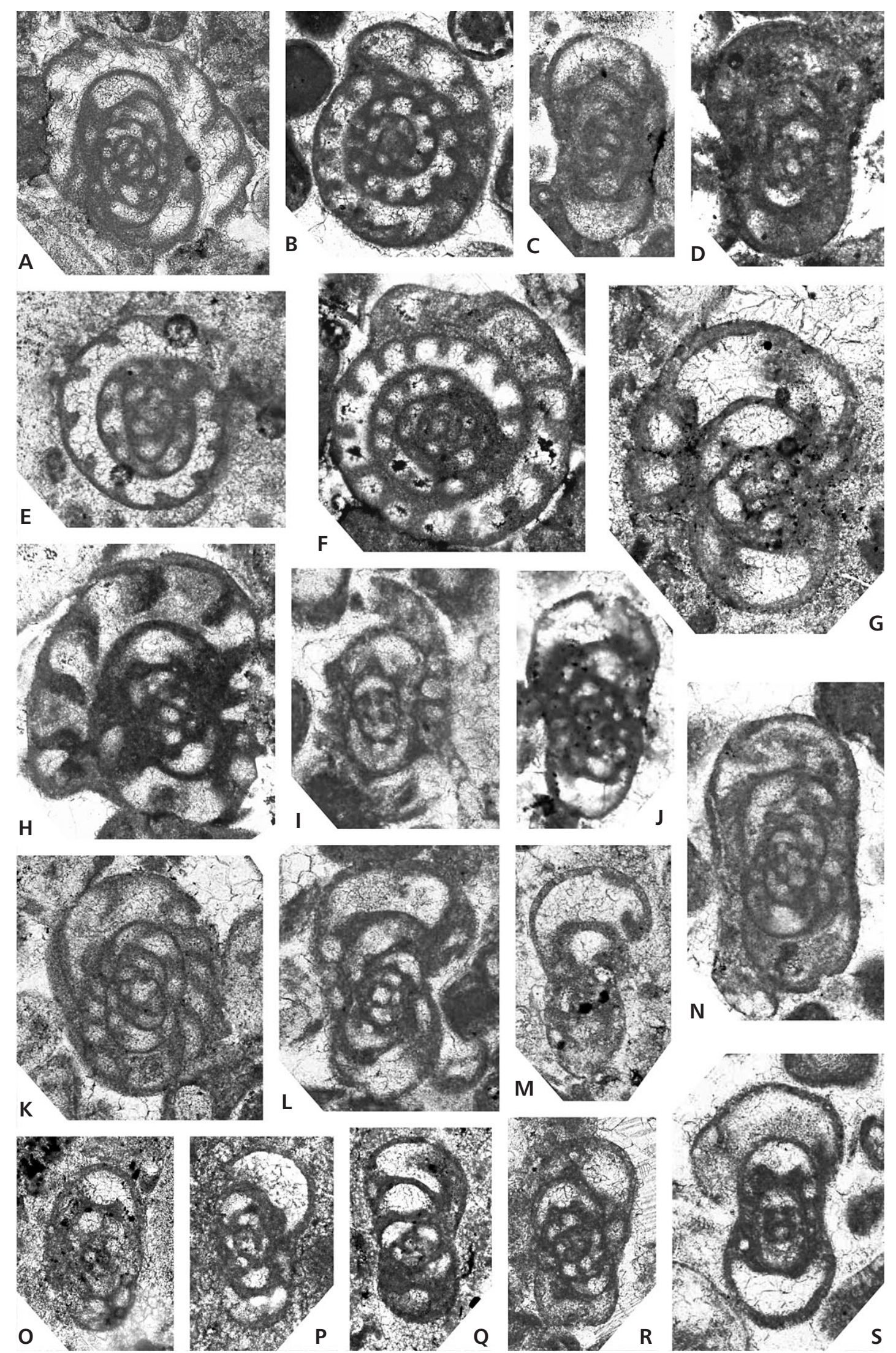

Figure 27. Typical foraminifers from Brown End in the Staffordshire Basin. For explanation see page 532. 

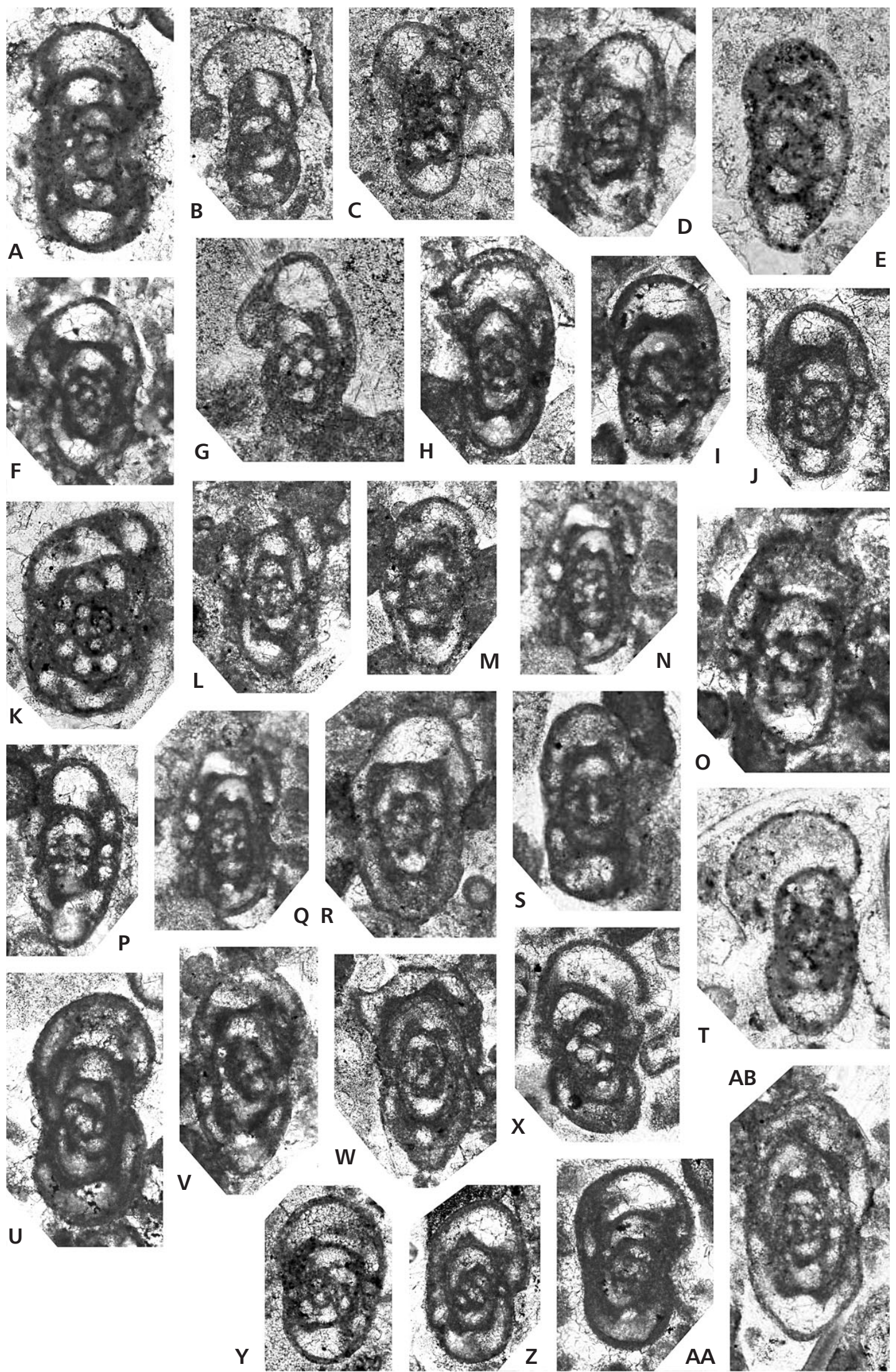

Figure 28. Typical foraminifers from Brown End in the North Staffordshire Basin. For explanation see page 532. 
Kabanov 2009, Westphal et al. 2010). They contain a distinctive foraminiferal association comprising abundant Endothyra, Bessiella and the tournayellid genera Spinobrunsiina, Brunsia, Eblanaia and Spinochernella but are without stratigraphically important Eoparastaffella or Lysella. In the British Isles very similar foraminiferal associations were described by Riley (1995) from the Chadian stratotype in the Craven Basin and both foraminiferal associations and the bioclast compositional trends compare well with those described in the Dublin Basin (Kalvoda et al. 2011) and in the Oughterard area (Devuyst 2006) of Ireland.

On the contrary, Eoparastaffella, totally absent in the Tournaisian component of the Milldale Limestone at Ladyside Wood, is a most common genus in the photozoan inner ramp Gully Oolite. Endothyra, Spinobrunsiina and Brunsia occur both in the photozoan Gully Oolite (SWMS) and in the heterozoan facies of the Milldale Limestone (NSB) which indicates their wider facies range (Gallagher 1998; Hance 1979, 1988; Hance et al. 1981; Herbig \& Mamet 1994; Mamet \& Armstrong 1972; Mamet et al. 1993). Eoparastaffella evidently inhabited only very shallow water parts of the inner ramp which is in accord with the ecology of descendant Staffelloidea (Vachard et al. 2010). This is also evident in Belgium where in basinal settings Eoparastaffella occurs in platform derived tempestites (Devuyst 2006, Lees 1997).

It is generally accepted that the most important factors controlling the distribution of benthic foraminiferal assemblages are trophic level and oxygen level (Jorrisen et al. 1995, 2007; Van der Zwaan et al. 1999) not depth. The occurrence of individual species thus indicate a certain level of organic flux to the sea floor and redox regime rather than a specific water depth (De Rijk et al. 2000, Van der Zwaan et al. 1999). The presence of heterozoan associations in shallow water Tournaisian limestones in British Isles instead of oligotrophic photozoan ones (Wright \& Faulkner 1990) may indicate higher nutrient levels (Carannante et al. 1988, Schlager 2003, Westphal et al. 2010). This interpretation is corroborated by the presence of foraminiferal genera Eblanaia or Spinochernella which are widespread in facies with higher nutrient and lower oxygen levels at the T-V boundary in the west and south facing ramps of the North American part of Laurussia (Ahr \& Stanton 1994, Armstrong \& Mamet 1988, Brandley \& Krause 1997, Mamet et al. 1986) and in dysoxic facies at the at the middle/upper Tournaisian boundary in Belgium (MFZ4) (Conil et al. 1991, Saltzman et al. 2004).

In summary, the compositional trends of foraminiferal associations are closely tied to the variations in the composition of skeletal debris. The Endothyra, Spinobrunsiina, Brunsia, Spinochernella, Bessiella, Eblanaia assemblage is associated with the dominance of heterozoan skeletal debris of the cool water factory (C factory) (Schlager 2005) which follows the mud-mound factory (M factory) of the Waulsortian and dominates in the latest Tournaisian in British Isles (Waters et al. 2009, 2011). The increase in photozoan components at the T-V boundary interval is gradual and indicates the gradual transition to the tropical factory (T factory) (Betzler et al. 1999, Wright \& Burgess 2005). It contributed to the production of skeletal debris of a more diverse composition on carbonate ramps resulting in higher representation of photozoan component and the growing presence of Eoparastaffella in calciturbidites. The diachronous transition from the $\mathrm{C}$ to $\mathrm{T}$ factory connected with the entry of Eoparastaffella in the North Staffordshire Basin [occurring later in Ladyside Wood (earliest Viséan) than in the Brown End Quarry (latest Tournaisian)] illustrates the problems of high resolution biostratigraphy at this stratigraphic level very well.

Variations in the composition of the skeletal bioclasts and the foraminiferal associations are thought to have been influenced by 4 factors.

1) Nutrients. According to the nutrient limitation model of Wood (1993) the heterotroph-dominated skeletal composition might be indicative of mesotrophic conditions. It is tempting to imagine a possible link between nutrient excess and the remarkable development of Waulsortian mounds during the late Tournaisian in British Isles (Lees 1982, 1997; Wright \& Faulkner 1990) as well as the heterotroph-dominated overlying carbonates.

2) Ramp topography. Tournaisian ramp topography is characterized by low shallow water production and higher production in deeper water (Wright \& Faulkner 1990). Where the nutrient flux is higher, however, heterozoan facies can occur also in shallow water environment, which may have also been the case in the British Isles (Wright 1986, Wright \& Faulkner 1990). The higher representation of photozoan skeletal debris in Viséan times (Somerville et al. 1992, Strogen et al. 1996) appears to be connected with the change in ramp architecture (transition from homoclinal to distally steepened ramp) favouring wider extent of shallow water production.

3) Biotic factors. Substantially reduced biological fixation of skeletal carbonates followed the Devonian-Carboniferous extinction (Kalvoda 2002, Wright \& Faulkner 1990). The major Carboniferous phototroph constituent, green algae, widepspread in shallow water environments in late Famennian (Berchenko 1981, Bogush et al. 1990, Matyja 2009) became quite rare in the Tournaisian regaining wider distribution only at the end of this stage (Devuyst 2006, Kalvoda et al. 2011).

4) Eustasy. Sea-level rise after the platform exposure during preceding sea-level fall may reconfigure the carbonate production portfolio owing to the different aggradation potential of the $\mathrm{C}$ and $\mathrm{T}$ carbonate factories during start-up, catch-up and keep-up phase of platform growth. The compositional data of the Ladyside Wood and 
Brown End sections suggest that heterotrophic assemblages (C-factory) shed their skeletal material into the basin continuously, even in the early Viséan start-up phase (cf. Schlager 2005, p. 25) above the T-V sequence boundary (Bábek et al. 2010). They became replaced by rapidly aggrading photozoan (T-factory) assemblages, dominated by dasyclads, during the following catch-up and keep-up phase.

It is apparent that some factors may have been interconnected and even though the resulting state represents a response to complex interactions, the contribution of single factors was differentiated. Nutrient flux may influence both the production on ramps and the distribution of calcareous green algae and thus appears to be the most important factor. The most common explanation of higher nutrient flux is upwelling (Carannante et al. 1988, Schlager 2003, Westphal et al. 2010). Although upwelling is not common in intra-cratonic basins it may have been supported by monsoonal system (James et al. 2004) assumed over southern western Laurussia during the studied time interval (Wright 1990, Wright \& Faulkner 1990). An increased nutrient flux might also have been caused by increased surface runoff connected with increased humidity (Wright 1990) and documented by the widespread distribution of late Tournaisian alluvial sediments in Great Britain at this time (Waters et al. 2009). The following decline in nutrient levels may be attributed to smaller river discharge from the diminishing relief of the Southern Uplands and Wales-Brabant High due to marine transgressions (Waters \& Davies 2006). An alternative explanation of variations in nutrient levels comes from Algeo \& Scheckler (1998) and Algeo et al. (2001) who argued that increased nutrient mobilization in the Late Devonian and Carboniferous was related to the evolution of deep-rooting vascular plants. In summary there may have been an interplay between different pathways of nutrient supply but the relative importance of these different pathways and their interactions with documented eustatic oscillations still have to be assessed.

\section{Conclusions}

The study of sections in the North Staffordshire Basin and South Wales-Mendip Shelf have so far revealed the richest foraminiferal associations at the T-V boundary in Western Europe. Among the stratigraphically most important Eoparastaffella species, the variable Eoparastaffella florigena group dominates and the index Viséan species Eoparastaffella simplex is quite rare. The FADs of other Viséan guides Eoparastaffella ovalis M2, Eoparastaffella asymmetrica and Eoparastaffella tummida and other bioevents such as the FAD of Lysella gaukensis close below the T-V boundary and the LAD of Eblanaia michoti close above the T-V boundary all help to define a refined high resolution biostratigraphic profile across the boundary interval and this facilitates a very good correlation with the stratotype area in South China. The study shows that the Eoparastaffella occurrences, and especially the evolutionary lineage from E. ovalis M2 to E. simplex, can be best followed in inner ramp photozoan dominated limestones. Their presence in carbonate turbidites therefore indicates supply from shallow water photozoan dominated areas. In the heterozoan dominated associations Eoparastaffella is absent or only very rare and a high representation of Tournayellidae and Bessiella is characteristic. The entry of Eoparastaffella is thus cryptic and the base of Chadian in the original definition is diachronous. This is well illustrated by the diachronous entry of Eoparastaffella at Brown End and Ladyside Wood in the North Staffordshire Basin. The Brown End quarry section with its excellent foraminiferal and good conodont record appears to provide a much better section for the definition of the base of the Chadian than the current stratotype at Chatburn bypass.

In the latest Tournaisian the facies development in British Isles shows some parallels with North America as Waulsortian buildups are followed by heterozoan dominated facies deposited on southward sloping homoclinal ramps of the Laurussian margin (Ahr \& Stanton 1994). This facies similarity is accompanied also by some similarity of the foraminiferal associations with abundant Eblanaia and Spinochernella). The change in the composition of the latest Tournaisian and Viséan foraminiferal associations follows the progressive increase in photozoan constituents typical for the ramp - platform transition. Compositional variations in foraminiferal associations are thus closely tied to facies variations indicating that further work is required on the ecology of these important foraminifer guides. This detailed sedimentological and microfacies study has provided important stratigraphic information by techniques appropriate for investigations of the T-V boundary elsewhere. Its value is, however, limited by the fact that most West European sections and the stratotype of the T-V boundary in South China contain only those foraminiferal bioclasts which have been transported to deeper environments (calciturbidites, tempestites). Moreover, the representation of Eoparastaffella ex gr. florigena and Eoparastaffella simplex in the studied sections shows that ecological distinctions have to be drawn in some cases at the species level.

Notwithstanding the problems discussed above the geophysical manifestation of the important 'mid Avonian' sequence boundaries provides a useful means of calibrating the detailed biostratigraphic changes at the T-V boundary. Their widespread recognition in Western Europe (Hance et al. 2002) and positive correlation with a $\delta^{13} \mathrm{C}$ shift recognized at the T-V boundary in the stratotype area of South China (Qie et al. 2011) support glacio-eustatic interpretations of the mid Mississippian sequences. 


\section{Acknowledgements}

This research was supported by grant project GACR 205/08/0182. Jiři Povolný is thanked for preparing numerous high-quality thin sections. Simon Hargreaves gratefully acknowledges the financial support provided by HEFCE for the pilot study of the Ladyside Wood section used in this work; a research-informed teaching project organised by Staffordshire University. The authors also acknowledge the assistance given by volunteers from GeoConservation Staffordshire in re-exposing the Ladyside Wood section for study. The paper has benefited from the helpful comments of Hans-Georg Herbig and Elena Kulagina.

\section{References}

Adams, A.E., Wright, V.P. \& Cossey, P.J. 2004. South WalesMendip Shelf, 393-476. In Cossey, P.J., Adams, A.E., PuRNell, M.A., Whiteley, M.J., Whyte, M.A. \& Wright, V.P. (eds) British Lower Carboniferous Stratigraphy, Geological Conservation Review Series 29.

Ahr, W.M. \& Stanton, R.J. JR. 1994. Comparative sedimentology and paleontology of Waulsortian mounds and coeval level-bottom sediments, lower Lake Valley Formation (lower Mississippian), Sacramento Mountains, New Mexico. Verhandlungen der Geologischen Bundesanstalt 50, 11-24.

Aitkenhead, N., Chisholm, J.I. \& Stephenson, I.P. 1985. Geology of the country around Buxton, Leek and Bakewell. Memoir of the Geological Survey of Great Britain, Sheet 111 (England and Wales).

Algeo, T.J. \& Schleckler, S.E. 1998. Terrestrial marine teleconnections in the Devonian: links between the evolution of land plants, weathering processes, and marine anoxic events. Philosophical Transactions of the Royal Society B 353, 113-130. DOI 10.1098/rstb.1998.0195

Algeo, T.J., Scheckler, S.E. \& Maynard, J.B. 2001. Effects of the Middle to Late Devonian spread of vascular land plants on weathering regimes, marine biota, and global climate, 213-236. In GENSEL, P.G. \& EdwARds, D. (eds) Plants Invade the Land: Evolutionary and Environmental Approaches. Columbia University Press, New York.

Armstrong, A.K. \& Mamet, B.L. 1988. Mississippian (lower Carboniferous) biostratigraphy, facies, and microfossils, Pedregosa Basin, southeastern Arizona and southwestern New Mexico. U.S. Geological Survey Bulletin 1826, 1-40.

Bábek, O., Kalvoda, J., Aretz, M., Cossey, P.J., Devuyst, F.X., Herbig, H.G. \& Sevastopulo, G.D. 2010. The correlation potential of magnetic susceptibility and outcrop gamma-ray logs at Tournaisian-Viséan boundary sections in Western Europe. Geologica Belgica 13(4), 291-308.

Baccelle, L. \& Bosellini, A. 1965. Diagrammi per la stima visiva della composizione Percentuale Nelle Rocce Sedimentarie. Annuali Dell'Universita Di Ferrara 9, 59-62.

BERCHENKO, O.I. 1981. Izvestkovyie vodorosli turneiskikh otlozhenii Donbassa. 71 pp. Naukova Dumka, Kiev.

Betzler, C., Reijmer, J.J.G., Bernet, K., Eberli, G.P. \& AnSELMETTI, F.S. 1999. Sedimentary patterns and geometries of the Bahamian outer carbonate ramp (Miocene-Lower Plio- cene, Great Bahama Bank). Sedimentology 46, 1127-1143. DOI 10.1046/j.1365-3091.1999.00268.x

Brazhnikova, N.E. \& VdovenKo, M.V. 1973. Rannovizeiski foraminiferi Ukraini. 296 pp. Naukova Dumka, Kiev.

Bogush, O.I., Ivanova, R.M. \& Luchinina, V.A. 1990. Izvestkovyie vodorosli verkhnego famena i nizhnego karbona Urala i Sibiri. 202 pp. Nauka, Novosibirsk.

Bozorgnia, F. 1973. Paleozoic foraminiferal biostratigraphy of central and East Alborz. Mountains, Iran. 185 pp. National Iranian Oil Company, Geological Laboratories 4.

Brandley, R.T. \& Krause, P.E. 1997. Upwelling, thermoclines and wavesweeping on an equatorial carbonate ramp: Lower Carboniferous strata of western Canada, 365-390. In JAMES, N.P. \& ClaRKe, J.A.D. (eds) Cool-water Carbonates. SEPM Special Publication 56.

BrenckLe, P.L. 1973. Smaller Mississippian and Lower Pennsylvanian foraminifers from Nevada. Cushman Foundation for Foraminiferal Research, Special Publication 11, 82 pp.

Brenckle, P.L. 2004. Late Visean (Mississippian) Calcareous Microfossils from the Tarim Basin of Western China. Journal of Foraminiferal Research 34(2), 144-164. DOI 10.2113/0340144

Buggisch, W., Joachimski, M.M., Sevastopulo, G. \& Morrow, J.R. 2008. Mississippian $\delta^{13} \mathrm{C}_{\text {carb }}$ and conodont apatite $\delta^{18} \mathrm{O}$ records - their relation to the Late Palaeozoic glaciation. $\mathrm{Pa}$ laeogeography, Palaeoclimatology, Palaeoecology 268, 273-292. DOI 10.1016/j.palaeo.2008.03.043

Burchette, T.V., Wright, V.P. \& FaulKner, T.J. 1990. Oolitic sandbody depositional models and geometries, Mississippian of Southwest Britain: implications for petroleum exploration in carbonate ramp settings. Sedimentary Geology 68, 87-115. DOI 10.1016/0037-0738(90)90121-9

Carannante, G., Esteban, M., Milliman, J. \& Simone, L. 1988. Carbonate lithofacies as paleolatitude indicators: problems and limitations. Sedimentary Geology 60, 333-346. DOI 10.1016/0037-0738(88)90128-5

Chisholm, J.I., Charsley, T.J. \& Aitkenhead, N. 1988. Geology of the country around Ashbourne and Cheadle. Memoir of the British Geological Survey, Sheet 124 (England and Wales).

Conil, R., Groessens, E., Laloux, M., Poty, E. \& Tourneur, F. 1991. Carboniferous guide foraminifera, corals and conodonts in the Franco-Belgian and Campine basins: their potential for widespread correlation. Courier Forschungsinstitut Senckenberg 130, 15-30.

Conil, R., Groessens, E. \& Pirlet, H. 1977. Nouvelle charte stratigraphique du Dinantien type de la Belgique. Annales de la Société Géologique du Nord 96, 363-371.

Cossey, P.J., Buckman, J.O. \& Steward, D.I. 1995. The geology and conservation of Brown End Quarry, Waterhouses, Staffordshire. Proceedings of the Geologists' Association 106, $11-25$.

Cossey, P.J., Gutteridge, P., Purnell, M.A., Adams, A.E. \& Walkden, G.M. 2004. Derbyshire Platform North Staffordshire Basin and Hathern Shelf, 305-364. In CosSEY, P.J., Adams, A.E., Purnell, M.A., Whiteley, M.J., Whyte, M.A. \& WRIGHT, V.P. (eds) British Lower Carboniferous Stratigraphy, Geological Conservation Review Series 29.

Conil, R., Longerstaey, P.J. \& Ramsbottom, W.H.C. 1980. Materiaux pour l'étude micropaléontologique du Dinantien de 
Grande-Bretagne. Memoires de l'Institute Géologique de l'Université de Louvain 30, 1-186.

ConIL, R. \& Lys, M. 1964. Matériaux pour l'étude micropaléontologique du Dinantien de la Belgique et de la France (Avesnois). Algues et Foraminifères. Mémoire de l'Institut géologique de l'Université de Louvain 23, 1-279.

Conil, R. \& NAum, C. 1977. Les foraminifères du Viséen moyen V2a aux environs de Dinant. Annales de la Société Géologique de Belgique 99(2), 109-142.

Delius, H., Kaupp, A., Muller, A. \& Wohlenberg, J. 2001. Stratigraphic correlation of Miocene to Plio-/Pleistocene sequences on the New Jersey shelf based on petrophysical measurements from ODP leg 174A. Marine Geology 175, 149-165. DOI 10.1016/S0025-3227(01)00115-3

De RiJk, S., Jorissen, F.J., Rohlinga, E.J. \& Troelstrac, S.R. 2000. Organic flux control on bathymetric zonation of Mediterranean benthic foraminifera. Marine Micropaleontology 40, 151-166. DOI 10.1016/S0377-8398(00)00037-2

DevuYst, F.X. 2006. The Tournaisian-Viséan boundary in Eurasia. Definition, biostratigraphy, sedimentology and early evolution of the genus Eoparastaffella (foraminifer). Ph.D. thesis, Catholic University of Louvain.

Devuyst, F.X., Hance, L., Hou, H., Wu, X., Tian, S., Coen, M. \& Sevastopulo, G. 2003. A proposed Global Stratotype Section and Point for the base of the Viséan Stage (Carboniferous): the Pengchong section, Guangxi, South China. Episodes 26, $105-115$.

Devuyst, F.X. \& Kalvoda, J. 2007. Early evolution of the genus Eoparastaffella (Foraminifera) in Eurasia: the 'interiecta group' and related forms, late Tournaisian to early Viséan (Mississippian). Journal of Foraminiferal Research 37(1), 69-89. DOI 10.2113/gsjfr.37.1.69

Doveton, J.H. 1994. Geologic log interpretation SEPM. Short Course Notes 29, 1-169.

Fewtrell, M.D., Smith, D.G., Clayton, G. \& Sevastopulo, G.D. 1981a. Discussion on the recognition and division of the Tournaisian Series in Britain. Journal of the Geological Society, London 137, 61-63.

Fewtrell, M.D., Ramsbottom, W.H.C. \& Strank, A.R.E. 1981b. Chapter 3, Carboniferous, 13-69. In Jenkins, D.G. \& Murray, J.W. (eds) Stratigraphical atlas of fossil Foraminifera. British Micropalaeontological Society Series, Ellis Horwood, Chichester.

GALlaGHER, S.J. 1998. Controls on the distribution of calcareous Foraminifera in the Lower Carboniferous of Ireland. Marine Micropaleontology 34(3-4), 187-211.

DOI 10.1016/S0377-8398(98)00006-1

George, T.N, Johnson, G.A.L., Mitchell, M., Prentice, J., Ramsbottom, W.H.C., Sevastopulo, G.D. \& Wilson, R.B. 1976. A correlation of Dinantian rocks in the British Isles. Special Report of the Geological Society of London 7, $1-86$.

Groessens, E., Conil, R. \& Hennebert, M. 1982. Le Dinantien du sondage de Saint-Ghislain. Stratigraphie et Paléontologie. Mémoire pour servir à l'Explication des Cartes Géologiques et Minières de la Belgique 22, 1-137.

HanCE, L. 1979. Révision micropaléontologique du Dinantien de Horion-Hozémont. Bulletin de la Société Belge de Géologie 88(4), 253-271.
Hance, L. 1988. Le Moliniacien du Synclinorium de Dinant (Belgique) de la région Dinantaise à la vallée de l'Ourthe. Mémoires de l'Institut Géologique de l'Université de Louvain 33, 1-91.

Hance, L. 1997. Eoparastaffella, its evolutionary pattern and biostratigraphic potential, 59-62. In Ross, C.A., Ross, J.R.P. \& Brenckle, P.L. (eds) Late Paleozoic Foraminifera: Their Biostratigraphy, Evolution, and Paleoecology and the MidCarboniferous Boundary. Cushman Foundation for Foraminiferal Research, Special Publication 36.

Hance, L., Hennebert, M. \& Overlau, P. 1981. Révision stratigraphique et sédimentologique du Tournaisien supérieur (Ivorien) et du Viséen inférieur (Moliniacien) de la vallée de l'Orneau (Belgique). Mémoires de l'Institut Géologique de l'Université de Louvain 31, 183-207.

Hance, L., Hou, H., Vachard, D., Devuyst, F.X., Kalvoda, J., Poty, E. \& Wu, X. 2011. Upper Famennian to Visean foraminifers and some carbonate microproblematica from South China - Hunan, Guangxi and Guizhou. 359 pp. Beijing Geological Publishing House, Beijing.

Hance, L., Poty, E. \& Devuyst, F.X. 2001. Stratigraphie séquentielle du Dinantien type (Belgique) et corrélation avec le Nord de la France (Boulonnais, Avesnois). Bulletin de la Société Géologique de France 172, 411-426. DOI 10.2113/172.4.411

Hance, L., Poty, E. \& Devuyst, F.X. 2002. Sequence stratigraphy of the Belgian Lower Carboniferous - tentative correlation with the British Isles, 41-51. In HiLls, L.V., Henderson, C.M. \& BAmber, E.W. (eds) Carboniferous and Permian of the World. Canadian Society of Petroleum Geologists, Memoirs 19.

Herbig, H.G. \& MAMET, B. 1994. Hydraulic sorting of microbiota in calciturbidites. A Dinantian case study from the Rheinische Schiefergebirge, Germany. Facies 31, 93-104. DOI 10.1007/BF02536935

Hladil, J., Geršl, M., Strnad, L., Frána, J., Langrová, A. \& SPIŠIAK, J. 2006. Stratigraphic variation of complex impurities in platform limestones and possible significance of atmospheric dust: a study with emphasis on gamma-ray spectrometry and magnetic susceptibility outcrop logging (EifelianFrasnian, Moravia, Czech Republic). International Journal of Earth Sciences 95, 703-723.

DOI 10.1007/s00531-005-0052-8

Ivanova, R.M. 2008. Nizhnyi karbon Borovskoi podzony Tyumensko-Kustanaiskoho progiba. Litosfera 2, 3-24.

James, N.P., Bone, Y., Kyser, T.K., DiX, G.R. \& Collins, L.B. 2004. The importance of changing oceanography in controlling late Quaternary carbonate sedimentation on a high-energy, tropical, oceanic ramp: north-western Australia. Sedimentology 5, 1179-1205. DOI 10.1111/j.1365-3091.2004.00666.x

Jorissen, F.J., De Stigter, H.C. \& WidMark, J.G.V. 1995. A conceptual model explaining benthic foraminiferal microhabitats. Marine Micropaleontology 26, 3-15.

DOI 10.1016/0377-8398(95)00047-X

Jorissen, F.J., Fontanier, C. \& Thomas, E. 2007. Paleoceanographical proxies based on deep-sea benthic foraminiferal assemblage characteristics, 263-325. In Hillaire-Marcel, C. \& de Vernal, A. (eds) Proxies in Late Cenozoic Paleoceano- 
graphy, Developments in Marine Geology 1, Elsevier, New York.

Kabanov, P. 2009. Benthic carbonate facies of the Phanerozoic: review and example from the Carboniferous of the Russian Platform. Stratigrafiya Geologicheskaya Korrelyatsiya 17(5), 41-59.

KaLvODA, J. 1986. Upper Frasnian-Lower Tournaisian events and evolution of calcareous foraminifera, close links to climatic changes, 225-236. In WaLLISER, O.H. (ed.) Global bioevents: a critical approach. Lecture Notes in Earth Sciences 8.

KalvodA, J. 1989. Tournaisian events in Moravia and their significance. Courier Forschungsinstitut Senckenberg 117, 353-358.

KALVODA, J. 2002. Late Devonian-early Carboniferous foraminiferal fauna: zonations, evolutionary events, paleobiogeography and tectonic implications. Folia Facultatis Scientiarium Naturalium Universitatis Masarykianae Brunensis, Geologia 39, 1-213.

Kalvoda, J., Bábek, O., Devuyst, F.X. \& Sevastopulo, G.D. 2011. Biostratigraphy, sequence stratigraphy and gamma-ray spectrometry of the Tournaisian-Viséan boundary interval in the Dublin Basin. Bulletin of Geosciences 86(4), 683-706. DOI 10.3140/bull.geosci.1265

Kalvoda, J., DevuYst, F.X., BÁBeK, O., Dvořák, L., RaK, Š. \& REZ, J. 2010. High-resolution biostratigraphy of the Tournaisian-Viséan (Carboniferous) boundary interval, Mokrá quarry, Czech Republic. Geobios 43, 317-331. DOI 10.1016/j.geobios.2009.10.008

Kulagina, E.I. 2011. Foraminiferovaya posledovatelnost v nizhnevizeiskikh otlozheniyakh razreza Verkhnaya Kardamovka na Yuzhnom Urale. Institut geologii Ufimskogo nauchnogo tsentra RAN, Geologicheskii sbornik 9, 205-218.

LEES, A. 1982. The paleoenvironmental setting and distribution of the Waulsortian facies of Belgium and southern Britain, 1-16. In Bolton, K., Lane, H.R. \& LeMone, D.V. (eds) Symposium on the Environmental Setting and Distribution of the Waulsortian Facies. El Paso Geological Society and The University of Texas at El Paso.

LEES, A. 1997. Biostratigraphy, sedimentology and palaeobathymetry of Waulsortian buildups and peri-Waulsortian rocks during the late Tournaisian regression, Dinant area, Belgium. Geological Journal 32, 1-36.

DOI 10.1002/(SICI)1099-1034(199703)32:1<1::AID-GJ715 $>3.0 . \mathrm{CO} ; 2-0$

MalaKhova, N.P. 1975. Foraminifery nizhnego vize vostochnogo sklona yuzhnogo Urala. Trudy Instituta Geologii i Geokhimii 112, 5-45.

Mamet, B.L. \& Armstrong, A.K. 1972. Lisburne Group, Franklin and Romanzof Mountains, northeastern Alaska. U.S. Geological Survey Professional Paper 800-C, 127- 144.

Mamet, B.L., Pinard, S.E. \& Armstrong, A.K. 1993. Micropaleontological zonation (foraminifers, algae) and stratigraphy, Carboniferous Peratrovich Formation, southeastern Alaska. U.S. Geological Survey Bulletin 2031, 1-32.

Mamet, B.L., Bamber, E.W. \& MacQueen, R.W. 1986. Microfacies of the Lower Carboniferous Banff Formation and Rundle Group, Monkman Pass map area, northeastern British Columbia. Geological Survey of Canada Bulletin 353, $1-93$.
Matyja, H. 2009. Depositional history of the Devonian succession in the Pomeranian Basin, NW Poland. Geological Quarterly 53(1), 63-92.

Postoyalko, M.V. 1975. Foraminifery i stratigrafiya rannego vize zapadnogo sklona Urala. Trudy Instituta Geologii i Geokhimii 112, 110-143.

Poty, E., Devuyst, F.X. \& Hance, L. 2006. Upper Devonian and Mississippian foraminiferal and rugose coral zonations of Belgium and Northern France: a tool for Eurasian correlations. Geological Magazine 143, 829-857. DOI 10.1017/S0016756806002457

QIE, W.K., Zhang, X.H., Du, Y.S. \& Zhang, Y. 2011. Lower Carboniferous carbon isotope stratigraphy in South China: Implications for the Late Paleozoic glaciation. Science China, Earth Sciences 54, 84-92. DOI 10.1007/s11430-010-4062-4

Ramsay, A.T.S. 1987. Depositional environments of Dinantian limestones in Gower, South Wales, 265-308. In MiLLER, J., AdAms, A.E. \& Wright, V.P. (eds) European Dinantian Environments. John Wiley and Sons, Chichester.

Ramsвоттом, W.H.C. 1973. Transgressions and regressions in the Dinantian: a new synthesis of British Dinantian stratigraphy. Proceedings of the Yorkshire Geological Society 39, 567-607. DOI 10.1144/pygs.39.4.567

Ramsвоттом, W.H.C. 1981. Eustacy, sea level and local tectonism, with examples from the British Carboniferous. Proceedings of the Yorkshire Geological Society 43, 473-482. DOI 10.1144/pygs.43.4.473

Rauzer-Chernousova, D.M., Bensh, F.R., Vdovenko, M.V., Gibshman, N.E., LeVEn, E.Y., Lipina, O.A., Reitlinger, E.A. \& Solovieva, M.N. 1996. Spravochnik po sistematike foraminifer paleozoya (endotiroidy, fuzulinoidy). 206 pp. Nauka, Moscow.

RIDER, M.H. 1999. The Geological Interpretation of Well Logs. 280 pp. Whittles Publishing Services.

Riding, R. \& WRiGHT, V.P. 1981. Palaeosols and tidal flat/lagoon sequences on a Carboniferous carbonate shelf: sedimentary associations of triple unconformities. Journal of Sedimentary Petrology 51, 1323-1339.

RiLEY, N.J. 1990. Stratigraphy ofthe Worston Shale Group (Dinantian), Craven Basin, north-west England. Proceedings of the Yorkshire Geological Society 48, 163-187. DOI 10.1144/pygs.48.2.163

RiLEY, N.J. 1993. Dinantian (Lower Carboniferous) biostratigraphy and chronostratigraphy in the British Isles. Journal of the Geological Society, London 150, 427-446. DOI 10.1144/gsigs.150.3.0427

RILEY, N.J. 1995. Foraminiferal biostratigraphy of the Chadian stage stratotype (Dinantian), Chatburn, northwest England. Bulletin de la Société belge de Géologie 103, 13-49.

Ross, C.A. \& Ross, J.R.P. 1987. Late Paleozoic sea levels and depositional sequences, 137-149. In Ross, C.A. \& HAMAN, D. (eds) Timing and Depositional History of Eustatic Sequences; Constraints on Seismic Stratigraphy, Special Publications, Cushman Foundation for Foraminiferal Research 24.

Saltzman, M.R., Groessens, E. \& Zhuravlev, A.V. 2004. Carbon cycle models based on extreme changes in $\delta^{13} \mathrm{C}$ : an example from the Lower Mississippian. Palaeogeography, Palaeoclimatology, Palaeoecology 213, 359-377. 
Schlager, W. 2003. Benthic carbonate factories of the Phanerozoic. International Journal of Earth Sciences 92, 445-464. DOI 10.1007/s00531-003-0327-x

SChlager, W. 2005. Carbonate sedimentology and sequence stratigraphy. 200 pp. SEPM Concepts in Sedimentology and Paleontology Series 8, Tulsa, OK.

Simonova, Z.G. 1975. Gumbeiskii i Ust'-Grekhovskii kompleksi foraminifer Magnitogorskogo sinklinoriya. Trudy Instituta Geologii i Geokhimii 112, 177-209.

Simpson, J. \& Kalvoda, J. 1987. Sedimentolgy and foraminiferal biostratigraphy of the Arundian (Dinantian) stratotype, 226-237. In HART, M. (ed.) Micropalaeontology of Carbonate Environments. British Micropalaeotological Society Series, Ellis Horwood, Chichester.

SkipP, B. 1969. Foraminifera, 173-255. In McKee, E.D. \& GutSCHICK, R.C. (eds) History of the Redwall limestone of northern Arizona. Geological Society of America Memoir 114.

SOMERVILLE, I.D. 2008. Biostratigraphic zonation and correlation of Mississippian rocks in Western Europe: some case studies in the late Viséan/Serpukhovian. Geological Journal 43, 209-240. DOI 10.1002/gj.1097

Somerville, I.D., Strogen, P. \& Jones, G.L. 1992. Biostratigraphy of Dinantian limestones and associated volcanic rocks in the Limerick Syncline, Ireland. Geological Journal 27, 201-220. DOI 10.1002/gj.3350270302

Strogen, P., Somerville, I.D., Pickard, N.A.H., Jones, G.L. \& FLEMING, M. 1996. Controls on ramp, platform and basin sedimentation in the Dinantian of the Dublin Basin and Shannon Trough, Ireland, 263-279. In Strogen, P., Somerville, I.D. \& JONES, G.L. (eds) Recent Advances in Lower Carboniferous Geology. Geological Society of London, Special Publication 107.

Stepanova, T.I., Kucheva, N.A. \& Postoyalko, M.V. 2008. Litologo-stratigraficheskaya kharakteristika nizhnevizeiskikh karbonatnykh otlozhenii nasseina r. Rezh (mironovskaya svita) na vostochnom sklone Srednegon Urala. Litosfera 5, 15-38.

Thibal, J., Etchecopar, A., Pozzi, J.P., Barthes, V. \& PocaCHARD, J. 1999. Comparison of magnetic and gamma ray logging for correlations in chronology and lithology: example from the Aquitanian Basin (France). Geophysical Journal International 137, 839-846. DOI 10.1046/j.1365-246x.1999.00828.x

Vachard, D., Pille, L. \& Gaillot, J. 2010. The Palaeozoic foraminifera: their systematics, palaeoecology and responses to the global changes. Revue de Micropaléontologie 53, 209-254. DOI 10.1016/j.revmic.2010.10.001

WAters, R.A. 1984. Some aspects of the Black Rock Limestone and Gully Oolite in the eastern Vale of Glamorgan. Proceedings of the Geologists Association 95, 391-392.
Waters, R.A. \& Davies, S.J. 2006. Carboniferous extensional basins, advancing deltas and coal swamps, 173-223. In BRENCHLEY, P.J. \& RAwson, P.F. (eds) The Geology of England and Wales. The Geological Society of London, London.

Waters, C.N., Waters, R.A., Barclay, W.J. \& Davies, J.R. 2009. A lithostratigraphical framework for the Carboniferous successions of southern Great Britain (Onshore). British Geological Survey, Research Report, RR/09/01.

Waters, C.N., Somerville, I.D., Jones, N.S., Cleal, C.J., Collinson, J.D., Waters, R.A., Besly, B.M., Dean, M.T., StePhenson, M.H., DAVIES, J.R., Freshney, E.C., JACKSON, D.I., Mitchell, W.I., Powell, J.H., Barclay, W.J., Browne, M.A.E., Leveridge, B.E., Long, S.L. \& McLean, D. 2011. A revised correlation of Carboniferous rocks in the British Isles. The Geological Society of London, Special Report 26.

WestPhal, H., Halfar, J. \& Freiwald, A. 2010. Heterozoan carbonates in subtropical to tropical settings in the present and past. International Journal of Earth Sciences 99, 153-159. DOI 10.1007/s00531-010-0563-9

Wood, R. 1993. Nutrients, predation and the history of reefbuilding. Palaios 8, 526-543. DOI 10.2307/3515030

WoRK, D.M. 2008. Secretary Editor's Report 2007-2008. Newsletter on Carboniferous Stratigraphy 26, 4.

WRIGHT, V.P. 1986. Facies sequences on a carbonate ramp: the Carboniferous Limestone of South Wales. Sedimentology 33, 221-241. DOI 10.1111/j.1365-3091.1986.tb00533.x

WRIGHT, V.P. 1990. Equatorial aridity and climatic oscillations during the early Carboniferous, southern Britain. Journal of the Geological Society, London 147, 359-365.

DOI 10.1144/gsigs.147.2.0359

WRIGHT, V.P. \& BURGESS, P.M. 2005. The carbonate factory continuum, facies mosaics and microfacies: an appraisal of some of the key concepts underpinning carbonate sedimentology. Facies 51, 17-23. DOI 10.1007/s10347-005-0049-6

Wright, V.P. \& FAULKNER, T.J. 1990. Sediment dynamics of Early Carboniferous ramps: a proposal. Geological Journal 25, 139-144. DOI 10.1002/gj.3350250205

Wu, X.T. 1982. Storm-generated depositional types and associated trace fossils in Lower Carboniferous shallow marine carbonates of Three Cliffs Bay and Ogmore-by-sea, South Wales. Palaeogeography, Palaeoclimatology, Palaeoecology 39, 187-202. DOI 10.1016/0031-0182(82)90022-0

Zwahn, G.J. van Der, Duijnstee, I.A.P., DulK, M. DEN, ERnst, S.R., JANNIK, N.T. \& KouwENHOEN, T.J. 1999. Benthic foraminifers: proxies or problems? A review of paleoecological concepts. Earth Sciences Review 46, 213-236.

DOI 10.1016/S0012-8252(99)00011-2 